\title{
ZUR LEXICOLOGIE UND GRAMMATIK DES ALTWESTFRIESISCHEN. ${ }^{1}$ )
}

Baerlioed, -liôde. S. u. flyûscâper fussnote.

Bêde, beithe in Hueerso ma een knappa iefta een fâmna ti bêde ieft iefta nimpt bùta rêde des mondes ende sibsta eerwena, dî haet wrberd hundert merk $\mathrm{W} 104,32 ;$ Jef di mond dae

1) Die hier verwanten abkiirzungen sind folgende:

$\mathrm{W}$ (nach $\mathbf{v}$. Richthofens $\mathrm{Wb}$.) $=$ der in den Rechtsquellen gegebene abdruck der Cölner (oder Anjumer) incunabel (s. v. R.'s Rq. s. XXIV).

$\mathrm{H}=$ die im 2. teil von De Haan Hettemas Oude Friesche wetten (Leeuwarden, Suringar, 1851) herausgegebene hs. des Jus municipale Frisonum (früher im besitz des herrn P. Wierdsma zu Leeuwarden, s. Rq. s. XXV, dann von $\nabla$. Richthofen angekauft, jetzt im besitz von $\nabla$. R.'s sohn, ober-regierungsrat a. d. Karl freih. v. Richthofen); citiert nach den seiten in Hettemas ausgabe.

S (nach v. R.'s Wb.) = die zweite hälfte der eben genannten hs., insofern dieselbe bei Schwartzenberg abgedrnckt und hiernach von v. R. in seine Rq. aufgenommen ist (s. Rq. XXV).

$J=$ die von De Haan Hettema besorgte ausgabe (Leeuwarden, Schetsberg, 1834) der hs. der Jurisprudentia Frisica (friher im besitz von P. Wierdsma, s. Rq. XXVI, dann von v. R. angekauft, jetzt im besitz von dessen sohn); citiert mit zwei zablen, von welchen die erste den titel, die zweite den paragraphen angibt.

Sch = Schwartzenberg, Groot placaat- en charterboek van Vriesland (Leeuwarden, Willem Coulon, 1768), 1. teil; citiert nach den seiten.

$\mathrm{Ag}=$ Oorkonden der geschiedenis von het st. Anthonij-gasthuis te Leeuwarden, door de voogden dezer stichting uitgegeven, 1. teil; citiert nach den seiten.

GJ $=$ Woordenboek op de (im anfang des 17. jahrhunderts verfassten) gedichten en andere werken van Gijsbert Japicx, zamengesteld door E. Epkema (Leeuwarden, Joh. Proost, 1824).

$\mathrm{Bl}=\mathrm{Ph}$. van Blom, Beknopte Friesche spraakkunst voor den tegenwoordigen tijd (Joure, R. P.Zijlstra, 1889); (nwfr. der jetzigen sprache

Beiträge zur geschichte der deutschen sprache. XIX.

23 
kŷnden toe beithe dêth, eer hiă toe iêrum commen sint, buta rêde der moedir, so ne mei diŏ dêda naet staen $\mathrm{H} 165$. Die zu vergleichenden parallelstellen hwaso een kneppa ieff $ı$ fâmna bynna aefta iêram to bôsta (zur ehe, s. Wörterb.) iout (gibt) ieffta nympt bata reed dis mondis and dis sibsta eerwa, dera wrberth ayder hondert merka Rq. 533, 36, und Jef di mond da kînden 10 a eft a (zur ehe) iout, eer hiă to hiâra iêrem commen sint, bâta rêde der môder, soe ne mei diŏ dêde naet staen. W 429, 12 führen $\mathrm{zu}$ der folgerung, dass bêde und beithe als synonyma von aefta und bôsta zu fassen sind. Es liegt nahe, für die beiden wörter an einen formell dem ahd. gebiuveda, gebueda habitatio entsprechenden prototypus *gibûithu mit der bedeutung 'cohabitatio' zu denken. Hieraus musste bei regelrechter entwickelung durch *-bê(j)ith-, *-bêth- die form mit $\hat{e}$, hingegen bei nichtsynkopierung des mittelvocals durch *.bê(j)ith-, *.bei(j)ith-, *-bei(j)ath- die form mit ei hervorgehen.

Dass nach oder während der wirkung des Sievers'schen synkopierungsgesetzes auf analogischem wege, d. b. durch beeinflussung von seiten der formen auf -ith- mit kurzer wurzelsilbe, das - $i$ - bei den formen mit langer wurzelsilbe bergestellt werden bez. erbalten bleiben konnte, ist zwar für das awfr nicht direct $\mathrm{zu}$ erweisen (alle belegten derivata mit -ith- begegnen ohne mittelvocal), wol aber indirect zu folgern aus den aofr. bildungen hanethe, thituethe neben benethe, lemithe, lovethe (s. Aofr. gramm. $\S 165 \alpha$, s. 136).

angehörende, doch bei Bl nicht verzeichnete formen citiere ich ohne weiteres).

Wenn in v. R.'s Wb. die belege und belegstellen ricntig angegeben sind, verzeichne ich die betreffende form in der regel nur mit hinzugefligtem $W$ und $S$.

Eine von dr. Buitenrust Hettema besorgte collation des v. R.'schen abdruckes mit dem texte der oben erwäbnten incunabel findet man Germ. 35, 1-34. Die vergleichung von De Haan Hettemas und Schwartzenbergs (bez. v. R.'s) ausgaben mit den beiden hss. konnte ich selber vor nehmen, indem mir diese schätze während einiger wochen von dem jetzigen besitzer gütigst zur verfügung gestellt wurden, dem ich hier auch öffentlich dafür meinen wärmsten dank zolle.

Die aus den collationen hervorgegangenen textberichtigungen verzeichne ich, falls sie nicht einer ausdrücklichen hervorhebung bedürfen, zwischen eckigen klammern hinter dem zu corrigierenden worte. 
Die genesis eines $e i$ aus $\hat{e}+$ vor $j$ entwickeltem anorganiscbem $i$ ergibt sich aus der parallelen erscheinung au, ou, eu aus $\hat{a}, \hat{o}, \hat{e}+$ vor $w$ entwickeltem $u$ (s. unten unter fliu(e)swerp und biliourva, causativ zu *biliva(n)). Andere belege für diese erscheinung werden die folgenden artikel in genligender zabl bringen. Wegen -the, d. b. -te, s. u. meit(h)ia. Wegen des schwunds des praefixes $\partial-<i-<j i-<g i$ - beachte man ausser den normalen participialformen gripen, beden, bonden, makat, meend, barnd etc. noch sîd 'gefäbrte, gatte' W, S, H passim, nâl 'genosse' W, H und J passim, nêde 'gnade' W, H 162. 168. 174, la(u)wa 'glaube' W, H und J passim, lyck 'gleich' W, S, $\mathrm{H}$ und $\mathrm{J}$ passim, mêne 'gemein' $\mathrm{W}, \mathrm{S}, \mathrm{H}$ und $\mathrm{J}$ passim, stênte edelgestein W, fêre 'nutzen' W (ahd. kifuori), noech 'genug' $\mathrm{H}$ 73, noeglyck, nôgelyck 'genügend' J passim, moede 'einwilligung' W 392, 22. 28. H 41 (mhd. gemuote), heynde und inlende (s. zu biriind), lenth (s. s. v.) etc.

Bigrêta. S. unten zu grêta.

Bihânet, -hâniget. S. zu hâna.

Byhellet 'ubervorteilt' in Dat een baer (vergleich) is folmachtich, al weer dat seeck, dat een man weer byhellet ende wr den haldeel (die hälfte) dis gueden [guedes]; ende al vêren dŷr fônden ênighe breuen jeff instrumenta, deer ma mede bywîsa mochte, dat hij bydrein (betrogen) weer in der soen, allîckwal so schel dy $\breve{o}$ soen in her macht blywa $\mathrm{J} 21,34$. Vgl. für dieses byhellet mnd. behalen 'ubervorteilen' und beachte wegen der form mit -ell- das simplex hellet 'bolt' $H 33$ (nwfr. hellje 'bolen' GJ) neben halia, -ien, -eth, -ed W, S, H passim, J 42, 3. 50, 41. 59,1 ; bytellia, -ie, -et etc. 'bezahlen' W 401, 12. Sch 520. 608. 609. J 1, 37. 2, 6. 8. 14, 2. 15, 39. 17, 16. 21, 36. 22, 1. 24, 10. 25,36. 27, 10. 32, 22 etc. ( $(\mathrm{w} w \mathrm{fr}$. betellje GJ) neben bitalia, -ie etc. S, Sch 394. J 43,13; doch dwalia J 1, 1 (nwfr. dwaelje GJ); wrbalede 'verschleuderte' J 50,41 (vgl. aofr. urbalia, Aofr. gr. $\S 299)$. Die zweierlei formen hängen selbstredend mit der verschiedenbeit der suffixe -ia,-iath etc. und -ath, -a, -ade etc. zusammen, und weil das $-a(-)$ schwerlich fur die genesis des -ellverantwortlich zu machen ist, muss die neubildung der einwirkung des zu $j$ gewordenen $-i(j)$-zugeschrieben werden.

Dieselbe erscheinung (also junge durch $j$ hervorgerufene dehnung und afficierung des $a \mathrm{zu} e$ ) ist auch bei den schwachen 
verben 2. klasse mit $r, k$ und $g$ (spir.) im auslaut der wurzelsilbe zu beobachten:

(wr)werria, -ie curare, servare $\mathrm{H}$ 36. 75. J 39,3. Sch 647 neben (bi)waria, -ade, -at etc. W, $\mathrm{H}$ 23. 31. 35. 48. 55. 58. 59. 61. J 2, 31. 26, 2. 6. 13. 28, 18. 39, 3. 46, 69. 50, 17. 58, 28. 72, 9. 81, 14 etc. (nwfr. bewearje mit ea aus $a$, doch bewerre praet., p. p. und imper. GJ; in einem nwfr. Zuidhoekschen dialekt bewaarje, Wassenberghs Taalkundige bijdragen 1,153); berria, -ie, -eth 'als schiedsmann einen vergleich $\mathrm{zu}$ stande bringen' J 21, 2. 32, 24. Sch 702. 704. 706 neben baria, -ie, -eth etc. J passim. Sch 609. 650. 74j; doch clarie, -ien J 21,26. 18, 2 ;

meckia, -ie, -ath etc. H 13. 29. 56. 57. 113. 129. 157. 166. 167. 168. 169. 172. J 1, 1. 18, 1. 11. 21. 21, 11. 19. 22, 13. 14. 23, 10. 24, 4. 25, 19. 26, 27. 28, 3. 8. 31,6. 46,36. 49,60 (nwfr. meitsje, s. unten zu meit(b)ia) neben makia, -ie, -ath, -ade etc. W, H 157. 161. 162. 174. 179. J 2, 5. 6. 14, 4. 17, 6. 19, 3. 21, 15. 25. 26. 22,1 . 6. 19. 20. 23, 2. 24,9 . 26, 12. 29, 2. 30, 17. 74, 2. 84, 4 etc.; smecket J 15,54, also smeckia (nwfr. smeitsje, s. zu mei(t) bia) neben smakia $\mathrm{S}$; weckien 'wachend' J 1, 13, also weckia (nwfr. weitsje, s. zu meit(b)ia); *reckia 'treffen' (zu folgern aus nwfr. reitsje, s. zu meit(b)ia);

beklegghet 'einklagt' J 3,10 neben klagia, -iath, -ath, -eth etc. W, S, H und J passim (nwfr. kleye mit kleggeste, -et und klegge praet., p. p. und imp. sg. GJ, und kleye mit klagest, -et, klage $\mathrm{Bl} \mathrm{155}$ ); *jeggia (zu folgern aus dem nwfr. praet. und p. p. jegge GJ) neben iagia, -et J 35,1. 71,5 (nwfr. jeye GJ und jeye mit jayest, -et, jage $\left.\mathrm{Bl} 155)^{1}\right)$; doch hagia, -iende, -et J 12, 25. 16, 4. 32, 9. 84, 12 (nwfr'. beheagje GJ); layhaden 'bestimmten' H 70 (vgl. aofr. lagia, Gramm. § 299).

Ob (bi)leckia 'anfechten' J 1, 35. 19, 8. S 503, 2. 37. 39 mit leckinghe $\mathrm{J} 19,8$ aus *lakia (vgl. laeckinge Sch 394) = mengl. lakien, mnl. laken vituperare (aus *lakôn) gleicher entwickelung ist, durfte fraglich sein, weil die form, wie aofr. leckia (Gramm. $\S 2$ ), durch anlehnung an leck vitium, vituperium $\mathrm{W}, \mathrm{S}, \mathrm{J} 65,14$ hätte entstehen können.

Durch contamination sind einige formen mit $a$ und gedehntem consonanten entstanden:

1) Vgl. noch nwfr. feye mit fegge etc. <*fagia, ${ }^{*}$ feggia $=$ mnl. vagen $<{ }^{*}$ fagôn. 
halled 'loolt' W 389, 27 (in W 389, 2.4 steht halla, -e fehlerhaft fur hallad, -ed; nwfr. in einem Zuidhoekschen dialekt halje, d. h. hallje, Wassenberghs Bijdr. 1, 156); bitallia, -ien, -ath etc. S 460, 12. Sch 495. 534. 537. 546. 590. 612.614. 617. 653. Ag 115; bivarria, warrien W 388,14 . J 26,6. 32,6. Sch 530. 537. 612. 709. Ag 159; barrie, -iane, -at etc. H 301. J 32, 22. Sch 690. 695. 705. 773. Ag 90. 114; mack(k)ia, -iet, -aden etc. H 431, 26. J 22, 15 (Hett. falsch meckkia). 20, 7. 15, 35. Sch 530. 535. 539. 671 (nwfr. makke etc., 8. zu meit(b)ia); sowie lackia, -et 'anfechten' Sch 334. 394 aus *lakia und leckya.

Wo dem consonanten ein andrer vocal als $a$ vorangeht, unterbleibt die dehnung:

bytilet 'erworben' J 33, 15, t(h)ilet, -ed 'zeugt' W, H 114, tilath 'zeugte' J 50,46; *boria (nwfr. boarje GJ); (bi)tigia, -ie, -ed etc. '(ver)klagen' W, S, H passim. J 43,3. 81, 15, artigia 'aufgeben' W (vgl. aofr. (bi)tigia, -tegia); pligia W, H 26. J 13, 11. 23. 45,1 ; fregia, $-a$, -ath etc. $\mathrm{W}, \mathrm{H}$ und $\mathrm{J}$ passim (nwfi. freegje GJ); hugiet 'in der erinnerung sind' J 15, 10 (nwfr. hurgje GJ).

Demzufolge ist das neben (bi)ieria, -et, -aden etc. W, H 64 . J 19, 9. 31, 1. 44, 12. 60, 9. 84, 22 und (bi)iaria, -et etc. W, H 53. 121. 132. 139. J 1, 11. 30. 2, 30. 19, 9. 25, 11. 27, 14. 41, 2. 82, 4. 84, 6 (mit $a$ aus $e$ vor $r$; nwfr. bejearje mit $e a$ aus $a$ ) begegnende bijerrien, -et etc. Ag 126. J 2,12. 21, 8. 28. 40, 3 als analogiebildung nach werria, berria neben waria, baria zu fassen. (Vgl. ausserdem bijarria, bijaerria Sch 737. 537 als mischformen aus bijaria und bijerria).1)

Byhyndiget 'beschimpft'. S. zu hyndicheed.

By câpet in Hwêrso eên man een caep dêth jeffta een seeck byjowt ende hyt dŷrney bynisa mey, dat hij den helfft jeffta deer wr byclôket sê off bycâpet (tubervorteilt) in da caep..., so toer hijt naet halda ende hyt aeg ney da riacht naet to staen $\mathbf{J} 32,11$. Die hier zu tage tretende bedeutung von $b y$ - 'zu eines schaden' (wegen der bedeutung des simplex vgl. got. kaupôn,

1) Das $r r$ in dem zu bejearje gehörenden praet. bejearre bei G. Japicx beruht nur auf eigentümlicher schreibung des dichters; vgl. bespoarre, folgge, mielle, plijgge, traeppe etc. praet. zu bespoarje, folgje, mielje, plijgje, traepje und s. auch $\mathrm{Bl} 139$. 
ahd. choufôn negotiari, mercari) begegnet auch im age. bi-, benéotan, as. biniotan 'berauben' (eig. zu eines andern schaden néotan, niotan nutzen ziehen), im ags. bi-, bedólan, as. bidêlian 'berauben' (eig. zu eines andern schaden eine teilung machen), awfr. bedêla 'bei der teilung ubervorteilen' (p. p. bedeeld in Hwaso wirth oneerwet off bydeeld ende byhînderet buppa da riuchta trymdeel J 46,29), im ags. berćdan 'berauben' (eig. zu eines schaden rat erteilen, durch falschen rat tbervorteilen) und im awfr. biclôkia 'betrtigen, ubervorteilen' (s. unten sub voce).

Dem bycâpet entspricht das im gleichen sinn verwante mnd. bicoft, bi-, bekofft (s. Schiller-Lübben unter bekôpen) und mnl. becoft (s. Mnl. wb. 1,811), nnl. bekocht.

Byclôkia. Die bedeutung 'betriggen, ubervorteilen' ergibt sich aus der im vorigen artikel citierten stelle sowie aus Hnaso des (lies der) behelplicheed des riachtes braka wil als restitutio in integrum, dat is een weerrekrygingh [neerrêkynghe] des gueden

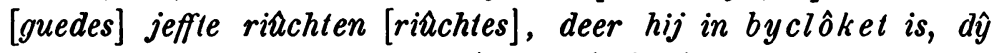
schel twŷr ponten to fara bewîsa... (primo) da mynre aldheed, deer hij dâ in was, da hij byclôket nârd... (secundo) dalter byclôket is jeffle bydreyn (betrogen) jeffta datter foerlern haet,

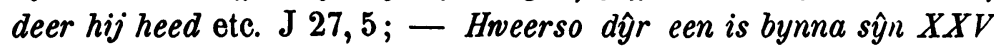
jeer byclôket ende hij dal benŷsd haet bêde dyoّ joget ende da byclôkinghe, so schel dij riuchter dis provincie him helpa mey da byhelplickheit des riuchtes, dat is hij schil hem wederjaen (wider in den besitz stellen) ende bringhen [bringhe] in $d a$ gueden ende riuchten, deer hij in der mynra aldheed byclôket is $\mathrm{J} 27,8$ (s. auch $\mathrm{J} 27,13$ ); - Jeff dyr sonderlinghe dat ferdban (die gerichtliche bestätigung des kaufs von immobilien) ginzen $w \hat{y} r$ jeens (gienge gegen, eintrag täte) veduen jeffta wêzen [wêsen] ende helliga tzercka, hwant weerl seeck, dat wedue off weesken off tzercke wêre behâniget (geschädigt, s. unten $\mathrm{zu}$ hâna) off byclôket myt da ferdban, so is dij riuchter altŷda, hij sê gâstlick of [off] wralsck, dat ferdban wanmachtich to riachten $\mathrm{J} 31,10$.

Vgl. auch mnd. beklôken circumvenire (Schiller-Lubben i. v.).

Mit by- 'zum behuf' begegnet unser compositum hingegen im sinne von 'seine klugheit zum vorteil einer sache gereichen lassen, fur etwas sorge tragen' in $\mathrm{J} 26,13$ und 14: '(wenn die vormunder die interessen ihrer mundel nicht, wie es sich gehört, wahren) so mey mes (man sie) allŷcvel staen lêla, offsette 
[offsetta] ende nyma oer monden off foersprecken to herre (der mündel) secken to foerwarien off to byclôckien [byclôkien], deer (was) hyaere [hyâre] oengeet' - 'een man in sŷn wŷfs secken, deer (wenu) hij naet byclôcket [byclôket], deer (was) her oengeet'.

Bilêva (oder bileuwa?), causativ zu *bilîva(n).

Biliouwa, causativ zu *biliva(n).

Fur altes $o, \hat{o}$ und $a+v($ d. b. $\delta)$ erscheint im awfr. $o u+$ $w$ und zwar durch die folgenden entwickelungsprocesse. Nach $o, \hat{o}$ und $\hat{u}$ wurde $v$ zu $w$, d. b. die bei der aussprache besagter vocale stattfindende lippenrundung und wölbung der hinteren zungenrlickenhälfte teilte sich der articulation des aus $f$ hervorgegangenen stimmhaften spiranten mit und wandelte diesen bierdurch um in den labialen halbrocal; darauf erzeugte das neue $w$ zwischen sich und dem vorangehenden $o$ bez. $\hat{o}$ einen unorganischen $u$-laut, der mit diesem vocal zum diphthongen rerbunden wurde; das $\hat{a}$ aber gieng vor dem neuen $w$ (wie das $\hat{a}$ und der durch die accentverschiebung gedehnte zweite component des diphth. $i \hat{i}$ vor urspr. $w)^{1}$ ) in ou uber. Belege: ouwer 'ufer' H 31. 35; biprouneth H 165, biprouuet W 434, 11, prouwien $\mathrm{J} 84,11$, biproulic $\mathrm{J} 81,5$, biprouwinga $\mathrm{J} 81,5$ (w0nach auch onera, -irra, ouir $\mathrm{W}$, ower $\mathrm{H}$ 34.67. 106, biprowat, -eth, prowad, -et, -it $\mathrm{W} 429,11.437,7$. H 26.177. J 9,6, oenprouinga $\mathrm{W} 106,22$, prowia $\mathrm{J} 84,11$, biprowinge $\mathrm{J}$ passim mit oun $\mathrm{zu}$ lesen sind);

1) So in bo(u)wa 'bewohnen' W, H 167, trouvia, -ed J 47, 1. 15. 55. 84, 3 (as. trion), $\operatorname{tro}(u)$ we adj. W, S, H 69.142.304. J passim, tro(u)we subst. W 69, 12. 77, 11. 425, 2. 433, 28. H 69. 81. 93. 175. J 12, 3. 15, 32. 24, 4. 84, 20. 21. 27 (nwfr. troun[e]), brout 'er braut' Sch 603 und inbrowen (l. nîbrowen) 'frisch gebraut' Sch 511 (aus *brîth, *gibrunvan), brout(e) und bryouth bräu Sch 603. 671.511 (alıs *briathe), rio(u)wen, -a, -ath W, H 113. J 56, 2 (nwfr. rouve dolere; wegen des schwunds des ersten componenten nach liquida in dieser form und trouwe, brout, brout $(e)$ vgl. Aofr. gramm. $\S 24$ anm. 1 und beachte noch awfr. bilowid, unten 8. 357, bidrâged fallunt $J 86,8$, nwfr. bidraga fallere, lauwer potius und leeuwe caros, lecun 'lieb', unten s. 364 , slung, s. zu flineswerp, rogt, slogt, s. zu focht, flìs = awfr. flyus, s. zu fly ûscâper, groede 'narbe', s. unten s. 356, rôp, s. zu hliâp a), biriousiget 'bereut' $J$ 86, 10 (ags. hréonsian), io(u)we poss. H 119. 122. 128. 131. 134. 162. 167. 170.172.173, woneben jedoch auch $i(\hat{u})$ we $\mathrm{W}, \mathrm{H} 300-304$. J 19,3. 21, I durch anlehnung an das person. in. 
houne (dat. zu hof) $\mathrm{W} 396,17.26 .412,20.413,20$ und hou Ag 95. W 63,12. 77, 29. 389, 18. 19. 425, 12. Sch 545, how W 396, 9. 20. 27. 33. 412, 22. 426, 17. 20. J 3, 10. 15, 27. 50, 41. 76, 3. 4. 81, 23, hounes (gen.) J 75,6, houwade Sch 699, houned p. p. J 81, 14; lounet, louned 'gelobt, verspricht' Sch 706. J 84, 20. 27 (nwfr. launje 'loben', belaunje 'versprechen' mit au, worüber s. v. sauwen); prouest, prowest W 406,17. 459,8. H 67. 105. 314 (wo das ou, on als schreibung fur oun zu gelten hat); prowenda 'pfrunde' H 109. 110 (2 mal); ouer, over(-), ovir $(-)$ 'über(-)' W 29, n. 13. 395, 20. 396, 3. 413, 34. 417, 19. 433, 29. H 51. 71. 73. 119. 130 (d. h. ouwer $(-)=\mathrm{nwfr}$. ouwr GJ) ${ }^{1}$ ); ounen 'öfen' Sch $3944^{2}$ ) (nwfr. own GJ 507) ${ }^{3}$ ); nwfr. groun 'grob', das auf awfr. *grouwes, - $e$ etc. zu *grof binweist;

dusscoune ein stoss, der 'dûst'4) zur folge hat $\mathrm{S}$ (vgl. nwfr. schouwe 'stossen' = aofi. skuva, ags. scúfan) und das in dustscowes zu corrigierende diststowes $\mathrm{W} 476,25$, wozu d $d \hat{\text { stschou }}$ W 462, 2 als analogiebildung (aus*.sk̂f); sowie die aus nwfr. dou 'taube', stouwe 'stieben', strou 'kuchen' zu folgernden awfr. formen *doune, *stoune (aus *stuva(n), das neben ahd. stioban steht, wie aofr. skuva neben ahd. skioban), *strouwe (mnd. struve 'kuchen') ${ }^{5}$ ); [nach GJ galten auch sch0we, st $\hat{n}$ 'e mit $\hat{u}$ aus den flexionsformen *schift, -st etc. und dun (un als schreibung fur $\hat{\imath})$ aus der apokopierten form, deren auslautend gewordenes $w$ durch $a$ absorbiert wurde; vgl. auch das seinem $d$ zufolge als entlehnung zu fassende $d r \hat{\imath}$ 'traube' (die fries. form musste nach ahd. $d r \hat{a b a}$ mit $t$ anlauten) mit $\hat{u}$ wie in $d \hat{u}] .{ }^{6}$ )

1) Die normale awfr. form ist $\operatorname{ar}(-), \operatorname{vr}(-)$ (nwfr. $\hat{\operatorname{ur}}[-])=$ aofr. $\operatorname{ar}(-)$ für *uvr (Aofr. gramm. $\S 116 \beta$ ).

9) Sch 603 steht obenen, das der heransgeber vermutlich falsch las für ouenen.

s) Daneben auch nwfr. unne (d. h. ane) mit $\hat{u}$ für *uv (wie in $\hat{u}(-)$, 8. oben note 1) aus *uvn.

4) Die etymologie des wortes ist mir dunkel. Was $\S 42$ der Aofr. gramm. darüber bemerkt worden ist, ist nicht zulässig, weil das $d$ des mnd. donen $=$ bair. donen strotzen (Schmeller $1^{2}, 515$ ) auf $p$ zurückgeht.

s) Vgl. noch das offenbar aus dem awfr. entlehnte im aofr. $E^{3}$ begegnende hourve baube $=$ ags. hífe.

6) Statt drûven uvae GJ steht in Wassenberghs Taalkundige bijdragen 1,167 droen mit oe als nicht etywologischer schreibung für $\hat{u}(n)) e$ (das zeichen oe diente nämlich zur darstellung des aus $\hat{o}$ hervorgegangenen diphthongs $\hat{u} \partial ;$ s. auch unten zu grôya). 
Der entwickelung von $\hat{u}-v$ zu $\hat{u}-w$ zu $o u-w$ entspricht die von $i u-v$ zu $i u-n$ zu $i \hat{\imath}-n$ zu iou- $n$ ( $w$ aus $v$ ist älteren datums als die accentverschiebung, wie sich aus dem unten auf s. $363 \mathrm{f}$. uber $i o-w$ aus io-v bemerkten ergibt): dyoul, dyowl 'teufel' $\mathbf{J}$ 46, 48. 15, 62 fur *diounel.

Wie durch vorangehendes $o, \hat{o}$ und $\hat{a}$, $i u$, wurde altes $v(t)$ auch durch folgendes $0, \hat{o}$ oder $u$ der endung beeinflusst und in $n$ umgewandelt, und zwar nicht nur wenn der rocal von haus aus unmittelbar nach $v$ stand, sondern auch (was für die chronologie der erscheinung zu beachten ist) wenn derselbe durch synkope eines $j$ nach dem consonanten zu stehen kam. Dies geht hervor aus den zahlreichen formen

mit $a u-n$ fur altes $a$ oder $\hat{a}$ (aus $a i$ oder $a u$ ) $+v$ (mittelstufe $a-w$ bez. $\hat{a}-w$; das $w$ entstand in den flexionsformen mit $v$ vor $-o(-),-\hat{o}(-)$ und $-u(-)$ und konnte natürlich von hier aus in andere flexionsformen oder verwante bildungen eindringen)

und mit $i \hat{u}$ (wenn das aus ursprunglich tautosyllabischem $v$ hervorgegangene oder vor dem ubergang von i $i$ in iou tautosyllabisch gewordene $w$ durch den zweiten componenten des diphtbongs absorbiert wurde oder auch wenn die form sich an eine flexionsform mit analogisch entwickeltem $i \hat{\imath}$ vor $f$ anlehnte) oder iou- $w$ bez. iou (wenn das $w$ nach der genesis von iou tautosyllabisch wurde) zum teil fur altes $i$ oder $\hat{\imath}+v$ (entwickelungsgang: $i-n$ oder in bez. $\hat{\imath}-n-\{u-n$ oder $i u-i \hat{\imath}-n$ oder $i \hat{u}-i o u-w$ oder $i \hat{u}$ ), zum teil fur altes $e$ (= urspr. $e$ oder aus $a$ in geschlossener silbe) oder altes $\hat{e}$ (= geschlossenem $\hat{e}$ oder aus $\hat{a}^{e}$ oder auch durch umlaut aus $\hat{o}$, ai oder $\left.a u\right)+v$ (entwicklungsgang: $e-w$ oder $e w$ bez. $\hat{e}-w-e u+w$ oder $e u-$ $i \hat{\imath}+w$ oder $i \hat{\imath}-i o u-w$ oder $i \hat{\imath}$ ); (aus den flexionsformen mit altem $-o(-),-\hat{o}(-)$ und $-u(-)$ konnte die eine oder die andre der mittelstufen oder auch i $\hat{u}$ bez. iou in andre flexionsformen oder verwante bildungen eindringen). - Belege:

hauk 'habicht' J 72, 4 (vgl. ahd. habuh und s. uber das au noch unten s. 359);

naula 'nabel' W, S (vgl. ags. neafola und s. unten s. 359); das nach nwfr. laungje 'laben' (GJ) anzusetzende awfr. *laun(i)gia (ahd. labôn; das $w$ entstand in der 2. 3. sg. praes. ind. mit altem $-\vec{o} s(t),-\vec{o} t h$ und in den praeteritalbildungen mit altem $-\vec{o}-;$ wegen der endung -(i)gia vgl. unten zu bâna); 
nwfr. kaunelje 'durch disputieren zu ergrinden, zu ermessen versuchen' GJ $=$ awfr. * kauwelia, das in cauelya 'durchs loos verteilen' Sch 657 vorliegen kann (wenn hier nämlich nicht eine form mit $v$ gemeint ist, vgl. was unten s. 363 uber die schreibung aue bemerkt wird; wegen der bedeutungen 'ermessen' < 'abmessen' < 'durchs loos abmessen, verteilen' dieses denominativs $\mathrm{zu}^{*}$ kavola, -ela $=$ mnd. kavele 'loosstäbchen' = an. kafli 'stuick, teil' vgl. das Mnd. und das Mnl. wb.);

haud 'haupt' W, S, H passim. J 2, 29. 15, 3. 21, 4. 22, 3. 50,3. 58, 41 (ags. héufod; s. noch unten s. 359) ');

lauwa 'nachlass' W 422, 7. H 2.60. J 87,6. 7 (ags. láf; man beachte die alten pluralendungen ${ }^{2}$ ) -0 nom.-acc., $-\breve{o}$ no gen., -um dat.) mit launigia, -ien, lauwegia, -ath etc. 'nachlassen' $\mathrm{H}$ 32. 58. 117. J 87, 5. 6. W 422, 7. Sch 608. 647, lauuigiet J 50, 41, laugia, -ien, -alh, -el(h) J 45, 14. 50, 36. 41. H 58. 92. 134. Ag 111, lauvede W 73, 31 (wegen -igia, -egia s. unten zu bâna);

(by)rauwia, -ien, -eth etc. '(be)rauben' J 81, 14. 22. 83,1. H 92. Sch 536. 706. 720, woneben durch anlehnung rauve 'raube' (dat. sg.) H 91. 304, rauwinge Sch 601, rauwer J 81, 23 ;

lauwa 'glaube' Sch 706. 733. 772. H 26. 71. J 85, 1. 4 (as. gilôto; beachte die alten endungen -o nom. sg., -un acc. sg. $>$ durch ausgleichung histor. -a für -an) mit on-, mis-, wanlauwich J 51, 1. 81, 14. 15. 22. 23;

bliân, -en (bliuua) 'bleiben' W 429,27. 430,1.437, 7. Н 25. 165. J 81, 15. 84, 13. 22. S 483, 34. Sch 518, dryâna, -en (dryuua) 'treiben' H 20. J 1, 41. 82, 4. 5. Sch 590. 723. 741, scriawa, -ane, -en (scriuua) 'schreiben' H 25. 63. 82. 167. J 15, 27. 46, 72. 81, 15. Sch 740 (mit î für regelrechtes iou durch anlebnung an die analogisch entwickelten 2. 3. sg. *bliust, *bliuth, *driust, *driath, *scriust, scryùt(h) J 56, 9. 81,14; das $w$ entstand in der 1. sg. und dem pl. auf altes $-u$ bez. -oth) nebst durch anlehnung entwickeltem scriawer $\mathrm{J} 46,59$; und blyouen ger. J 18, 13, bliouwath Sch 601 , scrioua, to scrioun J 11,

1) Daneben auch haed $W, H$ und $J$ passim mit schwund des in den flectierten formen durch Sievers' synkopierungsgesetz mit dental znsammengestossenem $v$, wie in hede 'hatte' aus hevida.

2) Das nomen begegnet in den denkmälern als plur. tant, 
3. 4. 24,22 . $25,12.26,2$, woneben auch mit analogischem iou die 3. sg. scriout J 17, 2. 15. 32, 22. 56,6. 64,12 ${ }^{1}$;

die praeterita plur. dryown 'trieben' $\mathrm{J} 62,14$, scriouwen 'schrieben' H 168 und scrioun J 36, 10. 46,59 (aus *drivun, * scrivun) mit analogisch gebildetem praet. sg. ind. bliou Sch 723, scryou W 437,3. H 25. 80. 81; das praet. opt. blyow J 70, 3 und die p. p. bliouwen Sch 723, dryown J 18, 15. 32, 8. 33, 5 . 55,5 . 70, 4, scriounen $\mathrm{H}$ passim, scrioun $\mathrm{W}, \mathrm{S}, \mathrm{J}$ passim und scriûven H 300. 302, schrieun Sch 351, scriuen Sch 351, scrioen Sch 530. 553. 604. 609. 617. Ag 99. W 435, 25 (mit ioe für î vor $n$ vgl. unten s. $358 \mathrm{anm} .4$; î stammt aus der synkopierten form und konnte in derselben vor $n$ erhalten bleiben durch einwirkung von scrînen, das selber durch scriuen vor ubergang in scriouwen geschützt wurde); (wegen anderer praeteritaler bildungen s. unten s. $360 \mathrm{f}$ );

liûwes, -e vitae, corpori $\mathrm{Ag}$ 107. H 66. 241 (das $w$ entstand im gen. dat. pl. *lîvo, -um; î wurde vor übergang in iou geschuitzt durch analogisch gebildetes *liuf);

nwfr. riûwe (riềve) und riù 'reichlich' adv. GJ $=$ awfr. *riûwe, *riu (aus *rîvo adv. zu *rîvi = mnd. rîve 'reichlich'; synkopiertes *rî schützte *rîtve vor übergang in riouwe und *riune verhinderte den nach dem unten zu focht erörterten bei regelrecht phonetischer entwickelung eintretenden ubergang von auslautendem $i \hat{u} \mathrm{zu} i \hat{o}$; das nwfr. hat riù mit gekurztem $-u$ in schwachbetonter silbe, vgl. zu focht);

nwfi. rî̀(we) rastrum $=$ awfr. * $(h)$ rî̀(we) (aus *hrîva, flect. - un $=$ an. hrifa, $-u$ rastrum; * riuwe und synkopiertes *riu schutzten sich gegenseitig);

nyauwen nepotibus H 56 mit ungenauer schreibung fur nyouwen (vgl. weiter unten 8. 360 ; wegen io $(u) w a$ verb. und jouwe 'gabe' s. daselbst);

iuun 'abend' Sch 658 (nwfr. juwn, d. h. jûn GJ), snî̀n 'sonnabend' Sch 646 (mit sn aus *sunnan-, sonnen- oder *sennan-, *sinnan-), vorbistorisches aus ioend, iônd H 162. W, Sch passim (s. Wb.), sniônd Sch 469. 546. 680709 (nwfr. snioen GJ) zu

1) Das iu hat sich in nwfr. festgesetzt: blîuwe, driûwe, skriùwe sowie kliûwe 'klimmen' (mnl. cliven mit $i$ aus $\hat{\imath}$ ), wriùwe 'reiben' GJ und Bl 147. 
folgerndes *(sn)iund ') mit absorbierung des durch vocalsynkope tautosyllabisch gewordenen $n$ und jowen Sch 389, jownd, jound $\mathrm{H}$ 75. Sch 607. 692. 709. 712 (nwfr. joun $\mathrm{Bl} 22$ ), sniownd Sch 598 (aus *êvun und *êvund, vgl. ags. ćéfen und mhd. âbunt, mnl. avont; (sn)iûn statt rein phonetisch entwickeltem (sn)iôn durch anlehnung an *iawen, den prototypus zu jowen; wegen ewnd, sneuwend und snyennd s. unten s. 360 f.);

brî̀wen [briunes] epistolae H 301 ( $w$ und $i \hat{u}$, wie in liuwes, s. oben; vgl. noch unten s. $360 \mathrm{f}$.);

griouwa 'graf' H 30 (3 mal). 31 (s. noch unten s. $360 \mathrm{f}$.); io(u)wen praet. ind. und opt. (aus *jêvun, s. unten zu geva); gryowa [gryowe] 'einen einschnitt machen' (als chirurgische operation) J 58, 41, grioud 'geschnitten' W 464, 21 (aus *grêva(n) mit $\hat{e}$ aus $\hat{o}$, vgl. mnl. groeven 'einen einschnitt machen', mnd. grôvinge 'einschnitt', beides als chirurg. operation; das $w$ aus der 1. sg. und dem pl. praes. ind. auf altes $-u,-\breve{o} t h$; s. noch unten s. 361); nwfr. groede 'narbe' Bl 22 mit synkope des ersten componenten (s. s. 351 anm.) = awfr. *griôde (aus *grênthe fur *grêwithu 'schnitt');

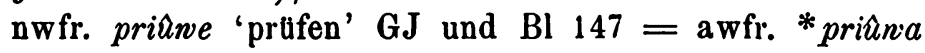
(aus *prêva $(n)=$ mhd. prüeven; erbaltung des $i \hat{u}$ wie in bli $\hat{\imath}$ wa etc.);

bilyowa 'gewähren' J 17,112) (aus *bilêva(n), vgl. ags. l'yfan permittere zu léaf permissio; nwfr. beljeune, $\mathrm{d}$. b. beliuwe, in Waatze Gribberts Brilloft 71 hat $i \hat{u}$, wie in bliuwa etc.);

lyo(u)wa, -en 'glauben' J 1, 20. 2, 37. 3, 9. 12, 5. 13,18. 25. 41. $15,40.45 .17,7.11 .17 .18 .24,13.67,6.84,10$, lyoud 'glaubt' J 81, 14 (aus *gilêva(n) = aofr. lêna, got. galaubjan; s. noch unten s. 361 und wegen des schwunds des praefixes oben s. 347) ${ }^{3}$ );

1) Vor $n d$ wurde $i \hat{\imath}$ zu iô: frioend, friônd $\mathrm{W}, \mathrm{B}$ und J passim (vgl. noch zu focht); -iund in festaiunde Sch 250 ist also als compromissbildung aus iuun und iônd zu fassen.

2) Hweerso een personna (ein geistlicher) off oers een man een breeff bysiglet foer een ôrem om beda willa, so schel hij selff dŷrom bidde, deert oengeet; ende schel ma om sŷn beda willa syglye, so schel hy dîy selm om sprecka ende by nessa, jeff hyt is een falsck breeff; ende dŷr schel ma neen syghel [sygel] den bodda (dem beauftragten) by ly o wa off neen bodda willa (keinem beauftragten zu liebe) breeff syglia.

8) Bei G. Japicx stehen. ljeauwe und (mit unurspr. -je) ljeaunje (d. h. ljéauwe, -je), die durch anlehnung an leauw(j)e glauben (d. h. léaourje, s. unten s. 361) für die alte form mit iou eintraten. 
liowe 'hinterlasse' J 46,12, lyout, lyowt 'hinterlässt' J 50, 5. 6. 87, 5. 7, liouweden 'binterliessen' H 94, lio(u)wa 'unberechnet lassen' W 469, 22. H 238, liowat(h) 'zurticklassen' W 426, 11. H 160 (aus * lêva(n)= ags. láfan; s. noch unten s. 361).

Die erkenntnis der erörterten lautentwicklung ermöglicht die deutung von bilyout $\mathrm{W} 395,10$, bilionet $\mathrm{W} 421,23$, bylion Ag 107 und bilowid W 436,12 (mit synkope des ersten componenten nach $l$ fur *biliowid, vgl. oben s. 351 anm.) als flexionsformen von biliouna (aus *bilêva(n), causativ zu *biliva(n)) $=$ 'einen oder etwas bleiben lassen, von einem oder etwas scheiden', und zwar in verschiedener verwendung 'einen im stich lassen, sich nicht mit ihm einlassen' - 'verlieren, nicht mehr haben' - 'aus etwas scheiden' - 'von etwas befreit werden' (vgl. mhd. scheiden von 'befreit werden'). Vgl. dat hi (der zum gerichtlichen zweikampf geladene, der die exceptio vorbringt, dass er zu einem andern gerichtsbezirk gehört) sîne schelta naemne schel, deer hi tô tiucht (auf den er sich bei seinem einwand beruft) ende sine nêsta bûren; soe aegh him sŷn aesga deer to dêlane, dal hyt op dae helgum wila (bezeugen) moet, hweer sŷn huis ende sŷn hof sê, hit ne sê dat him di schella milta tî̀ge inhalia (als zu seinem bezirk gelörend anerkennen, eig. zu sich holen) wil; iof di schella him bilyout (im stich lässt, also nicht inhalat), so aech hyt op da helgem to swaren ..., hweer sŷn huis ende sŷn hof sê; die parallelstelle $\mathrm{H} 47$ bat bilest [bilêft]; - Jef een man haet onaefte bern teyn (gezeugt) ende hi dan iout (gibt) gued minra iefta mâra, so môten da bern lawigia (das gut vererben) op hiâra bern al to da tredda; alsoe (auch) môten hiă lavigia op hiâra folbrôder ende op sŷn folsustera, ist dat hi $\breve{a}$ fan êne liue bern (geboren) sế; so hy $\breve{a}$ dan des (der erbfähigen nachkommen- und verwantschaft) ${ }^{1}$ ) biliowet (verlustig sind), so moet dat flotieflige gued (das den kindern als mitgift verliehene gut) weer in, aldeer hit is wt commen; die parallelstelle H 117 hat bilewath (mit $\hat{e}-v$ oder $e u-w$ ? s. unten s. 361); dat ick ... liânis (aus dem leben) byliow (scheide) ..., soe byspreck ick (vermache ich) etc.; vgl. daneben als hy des lywes byleweth (mit $\hat{e}-v$ oder eu-w ?), soe schilla da fiower schilde

1) Die parallelstelle in $\mathrm{H}$ hat statt des die lesart des taemes, die gewiss nicht die ursprüngliche ist, weil hier nicht nur von kindern und enkeln, sondern auch von geschwistern als erben die rede ist. 
rente ... faelle op dat convent $\mathrm{Ag} 102$; - Nâ (nachdem Octavian sich alle völker unterworfen hat) is al dy $\breve{o}$ wrâld truch Romes drêde (aus furcht vor Rom) wîges bilowid (befreit) ende toe mêna freda commen; die parallelstelle $\mathrm{H} 24$ hat bilewid (mit $\hat{e}-v$ oder $e u-w$ ?).

In betreff der in rede stehenden lautlichen erscheinungen möchte ich ferner noch einiges bemerken, und zwar zunächst, dass altes $-o(-),-\hat{o}(-)$ und $-u(-)$ der endung auf vorangehendes $v$ auch dann einwirkten, wenn die beiden laute durch einen consonanten von einander getrennt waren:

jowns 'gleichfalls' J 21, 17. 46, 45, onjown 'ungleich' J 21, 1 , jowncristen $\mathrm{J} 46,15.56^{1}$ ) (entstanden durch anlehnung an die aus altem *evno $=$ aofr. evna hervorgegangene form *ewno, also *wen $\mathrm{zu}$ *euren $\mathrm{zu}$ *îwen etc.; wegen euen, ewen s. u. s. 362); lio(u)wen 'becken' S 449, 7. 464, no. 19. 470, no. 1. H 228. 238 (das $w$ entwickelte sich in den pluralformen *lêvnu nom. acc. fur. *lêvinu ${ }^{2}$ ), *lêvno, -um gen. dat.; das subst. ist identisch mit mud. und mittelostndfränk. im Mnl. wb. und im Teuthonista verzeichneten loven (in lovenbecken), dessen urspribglicher wurzellaut mit rucksicht auf das $o$ der mittelostndfrk. form ${ }^{3}$ ) als langer (altem $\hat{o}$ oder au entsprechender) laut anzusetzen ist; wegen lewyn s. unten s. 361);

tio(e)le 'tafel' $\mathrm{H}$ 1. 2. 28. 29. 30. 68. Sch 346 (scluwaches fem.) mit $i \hat{o}$ für $i \hat{u}$ vor $l$ (wie auch sonst vor liquida und dentalem nasal) ${ }^{4}$ ) für *(île (aus *tevla, -un mit $e$ aus $a$, wio ags. $a$ in toefle ${ }^{5}$ ); wegen teula, $-e$ s. unten s. 361);

1) Das nämliche jow $(e) n$ steckt in der verderbten lesart owensens $=$ 'gleichfalls' in $\mathrm{J}$ 50,41: alzo he (der gatte, der ohne kirchliches ceremoniell sich verheiratet hatte) starff, wolde hij da bern to eerven habba and aefft makia, so was dat gued hyârens (gen. pl. des person.); nelthera (d. h. nelde+ere, wollte er nicht), so onteervader (enterbte er) se o wen sens.

2) Vgl. ags. ticcenu, mádenu, nietenu.

8) Altem in offener silbe stehendem $o$ entspricht im 'Teuthonista $a e$, $a i$, als schreibung für tonlanges $a$ : baide 'bote', baeven 'oben', aeven 'ofen', vaighel 'vogt', laeven 'loben', caicken 'kochen', caicker 'kocher' etc.

4) Vgl. fyoer, flect. fio(e)re W, S, H 28. 104. 115. 301, dyo(e)re 'teuer' $\mathrm{W}, \mathrm{S}, \mathrm{H} 90.113, \mathrm{~J} 11,4$. 80, 6. 86, 6, dioerthe H 24, slyôra 'steuern' J 60, 23, stioerde 'steuer' W, H 65, sio(e)ne, syoen 'gesicht' W, S, J 2, 14. 13, 2, syoen 'gesehen' $2,15.14,1.15,54.22,7.27,4.58,4$ l (vgl. u. zu fli ue swerp), scrioen 'geschrieben' (s. ob. s. 355). Wegen fyuer und der nwfr. formen s. zu focht.

5) S. auch Beitr. 5,65 . 
lîuwat(h), -elh 'lebt' H 32.55.60.94. J 87, 5, liûwade 'lebte' H 79, liûwet 'gelebt' H 112 (die afficierung entstand im praet. pl. ${ }^{*}$ livdun $=$ aofr. ags. lifdon; man beachte, dass die praeteritalen formen auf -ade, *ad, -et neubildungen sind, welche durch den zusammenfall der auf *-ais, *aith zurlickgehenden sufỉxe fur die 2. 3. sg. praes. ind. -as $(t)$, -ath mit -as $(t)$, -ath nach der 2. klasse hervorgerufen wurden; die erbaltung des $i \hat{u}$ vor $w$ ist die folge von anlehnung an das subst. *liuf, liûwes, $-e$, s. oben; wegen liwath etc. s. unten s. 363);

nwfr. stjuwn prora GJ, d. b. stjôn, = awfr. *stiuna (aus $*_{\text {stevno }}=$ ags. stefna; das vor $n$ nicht zu iô entwickelte $i \hat{\imath}$ erklärt sich als die folge der einwirkung einer doppelform *stiûmena);

nwfr. jouwer 'bafer' GJ mit verlust des anlautenden $h$, wie in joe(d) 'beute' (awfr. hioede), aus aw fr. *hiouwera (fur altes ${ }^{*}$ hevro $=$ ahd. habaro);

nwfr. thauwerje 'zaubern' GJ und towerie $\mathrm{J} 80,8$ (das ou der letzteren auf ein verb. * $\operatorname{toun}(\epsilon)$ ria hinweisenden form berubt auf einem in den flexionsformen mit altem $-\breve{o} s(t),-\breve{o} t h$, $-\breve{o} d(-)$ entwickelten $\hat{o}$, das $a u$ von awfr. *lauweria auf dem $\hat{a}$ der anderen flexionsformen; vgl. tuber $a$ und $\hat{o}$ aus $\hat{a}$ vor $w$ zu fliueswerp, trouwaden und sauwen; wegen des aw in tawerie $\mathrm{W}=a u-w$ oder $\hat{a}-v$ ? s. unten s. 363 );

nwfr. stjungje firmare, stabilire GJ, d. b. stiagje, und stjung firmus = awfr. *stiagia, *stîng (aus *stevgia, *stevges, - e etc. fur *stev(i)gia, *slev(i)ges, -e etc., vgl. mnd. mittelostndfrk. stevig firmus).

Aus dem ou-w, das auf ein durch dunklen endungsvocal hervorgerufenes $\hat{o}-w$ bez. $o-n$ fur $\hat{a}-w$ bez. $a-w$ zuruckgeht (s. die bei thaunerje erwähnten artikel), ergibt sich, dass in den oben verzeichneten wörtern mit au-n (au) diese lautverbindung aus den flexions- bez. doppelformen stammt, die kein $\breve{o}$ oder $u$ in dem flexions- oder ableitungssuffix hatten (wegen hauk, naula, haud, oben s. 353 f., vgl. mhd. habich ${ }^{1}$ neben ahd. habuh, ags. nafela neben neafola, ahd. houbit ${ }^{1}$ ) neben ags. héafod) und das $w$ auf analogischem wege erhielten.

1) Die hiernach anzusetzenden formen *havik, *havid mit nicht umgelautetem vocal durch anlehnung an *havuk, *hâvud. 
Als gegenstuck zu jenem ou aus $a$ erseheint ein durch die nämlichen factoren entwickeltes ou für $e$ (mittelstufe $o$ ) in nowa, nouua nepos J 11,6. 15, 27. 76. 47, 14. 49, 1. 2. 50,1. 4. 35. 57, 8. Rechtsqu. 461, no. 4; sowen 'sieben' Sch 670. S 385, 9. 456,13, sowenteen $\mathrm{S} 492,1$, soewentich Sch 670 (s. noch zu sa uwen). Das neben ersterer form stehende nyouwa (s. oben s. 355) muss demnach aus den flexionsformen mit altem *-wa(n) herrtibren, welche $n$ durch analogie annahmen. Im praes. io(u)wa, -ane, - $e$ etc. (s. unten zu ge va) und im subst. jo(u)we 'gabe' H 117. 178. 180. J 28, 8 (= ags. zifu) fielen die durch beiderlei lautentwickelung entstandenen bildungen zusammen: *gerwu 1. $\mathrm{sg}$. ind., -oth pl. zu *gowu, -oth $\mathrm{zu} *$ jowu oder -e, -oth oder -uth (mit $j$ für $g$ nach den anderen flexionsformen) zu *jouwu oder jouwe, *jouwoth oder jouwath, oder aber (wenn das $j$ schon fruheren datums ist) *jewu, -oth $\mathrm{zu} *$ jowu, -oth $\mathrm{zu} *$ jounu etc.; *gene, -en opt., *gewan, -ane inf. ger. zu *geuwe etc. oder *jeuwe etc. zu * giune ete. oder *jiune etc. zu *giûne etc. oder *jâne etc. $\mathrm{zu} *$ giouwe etc. oder jouwe etc., oder aber *jewe etc. $\mathrm{zu} *$ jeuwe etc.; *gewu nom. sg., -o, -um pl. zu *gomu etc. zu jowu etc. (mit analogischem $j$ ), oder aber *jenu etc. zu $*^{*}$ jeuwu etc.; ${ }^{*}$ gewa gen. acc. sg., -e dat. sg. zu *geun- oder *jeuw- etc., oder aber *jewa, -e zu *jeuw- etc.

In einigen formen mit altem $\hat{e}$ vor $w$ aus $v$ findet sich ausser iu oder iou noch eu, offenbar als die folge einer die normale weitere entwickelung verhindernden einwirkung von seiten der neben- oder flexionsformen mit lautgesetzlichem $\hat{e}$ vor $v$ oder $f$ :

ewnd 'abend' Sch 521, sneuwend 'sonnabend' Sch 608 neben jound (s. oben s. $355 \mathrm{f}$. und vgl. fur die anzusetzende nebenform mit $\hat{e}-v$ ahd. abant $=$ vorfries. * $\left.{ }^{2} v a n d\right)$; breunis, -e, -en 'briefes, $-\theta$ ' Sch 608. 609. 695. 745 neben briawes (s. 356); greuwa 'graf' W 412, 5. 24. 29. 413, 1. 6. 13. H 3. 25. 30. 34. 37. 38. 40. 45.58. $8 \overline{5}$, greuua W 389,34. 390, 1. 437, 5 neben griouwa (s. 356);

sowie die zu skriûne, blîne, driâne, kliûne, wriûne (s. oben s. 354 f.) gehörenden praeterita pl. und part. skreaun, bleaun, dreaun, kleaun, wreaun GJ und Bl 147 (eau als schreibung für diphth. $\left.e^{a} o^{u}\right)$, welche auf *screuwen (-un) 'schrieben', screuwen 'geschrieben' Sch 630.695. 745, *bleuwen '(ge)blieben', etc. binweisen (mit eu aus $\hat{e}$, das, aus dem praet. ind. sg. ent- 
lehnt, altes $i$ verdrängt hatte; wegen scriouwen etc. s. oben s. 355) ${ }^{1}$;

nwfr. leaunje 'glauben' GJ (mit unurspr. -je, wie oft im nwfr.) und leeuve Wassenberghs Taalkundige bijdragen 1, 152. 162 (wegen lyouwa 'glauben' s. oben s. 356);

nwfr. kleauwe 'spalten' GJ = awfr. *kleuwa (aus *klêva(n) $=$ mnl. kloven < $<$ klôtian).

Ob dieses $e u$ auch in all den mit $e w, e v$, eu geschriebenen belegen breuis, -e, -en W 388, 2. 402, 25. S 479,11. 489,15. J 8, 4. 13, 2. 17, 1. 4. 7. Sch 395. 532, brewis, -e Sch 337. 489. 496. 533. 537. 612. Ag 92, breve W 441, 31, grewa 'graf' W, $\mathrm{H}$ passim. J 1,10. 72, 2 etc., screve 3. sg. praet. ind. (s. unten zu geva), (bi)screuen W 427, 31. 430,34. Sch 395. 435. 547, screwen Sch 395. 462. 608, screven Sch 496, dreuen J 2,31, bilewen 'gestorben' $\mathrm{H}$ 95, oder auch in greweth, -edh, greuetd 'geschnitten' $\mathrm{S}$, greue 'wundarzt' $\mathrm{S} 443,16$ (vgl. oben s. 356 uber gryowe), lewa 'unberechnet lassen' S, bilewath, -eth, bilewid (s. $357 \mathrm{f}$.$) , lewyn,$ -en 'becken' W 15, 8. 464, 13. H 74, teula, e- 'tafel(n)' H 80. Sch 394 etc. vorliegt, durfte nicht ganz sicher sein, weil mit rücksicht auf die sonstige verwendung eines $n, v$ oder $u$ zur bezeichnung von $v$ und die möglichkeit der erhaltung im awfr. von formen mit $\hat{e}+v$ es nicht für unwahrscheinlich gelten kann, dass hier zum teil wenigstens brêve etc. gemeint sei.

Durch jungere anlehnung an flexionsformen mit erhaltenem $\hat{e}$ (vor $v$ ) entstanden ferner brieune H 69, grieuwa $\mathrm{H} \mathrm{34.} 38$. 119. 121. 122. 124. 125. 126. 127. 129. 136 etc., snyennd Sch 692 mit diphthong $i e, \operatorname{der}$ im $n w f r$. allgemein fur altes $\hat{e}$ eintrat und der mitunter in den älteren quellen fur $e$ oder $e e$ stehenden schreibung zufolge ${ }^{2}$ ) schon im awfr. im aufkommen

1) Man beachte auch das praet. sg. skreau, bleau etc. GJ und Bl für screeff W, H 25. 108. 157. Sch 530, "bleef etc. Die nwfr. preau, preaun, treau, treaun, reau, reaun zu prî̀ne 'prüfen', trî̀ve 'drücken, drängen' (got. preihan), riane 'reihen', sind selbstredend die resultate von neubildung, bei ersterem verbum veranlasst durch den zusammenfall des praes. priûne (aus * prêva(n), s. oben s. 356) mit skriûne etc., bei den anderen durch den zusammenfall des praet. pl. *thrêtunn, *rênun (ê für $i$ aus dem sg.) mit *skrênun etc. (daher auch triûne, riûne statt eines bei regelrechter genesis $z \mathfrak{u}$ erwartenden $t r \hat{i} j e, r \hat{i} j e)$.

2) Vgl. brief(f) H 30.110. J 30, 3. Sch 351.462. 464. 472.511. 541.604, priester H 62, Pieter H 162, hiet, -en praeter. H 163 . W 429, 28, hieta praes. 
begriffen war, $\mathrm{d} . \mathrm{h}$. durch eine diphthongische aussprache $\hat{e} \partial$ (mit geschlossenem, dem $\hat{\imath}$ naheliegendem $\hat{e}$ als erstem componenten) vorbereitet wurde. Fur brieue, -en H 98. 106. J 81,14 (Hett. falsch breuen). 23. Sch 552. 591. 605, brievis Sch 553, grieva H 36. 53. 131. 135. 136 etc. gilt das oben uber breue etc. bemerkte.

Ein dem eu in ennd etc. zu vergleichendes eu ist auch fur formen mit altem $e$ nachzuweisen: nwfr. kreauwelje 'kitzeln' GJ $=$ awfr. $*$ kreuwelia (aus $*$ krevlia $=$ mnd. mnl. crevelen 'kitzeln') 1) und nwfr. kreeuwe 'kitzelig sein, streit anfangen im kinderspiel' Wassenberghs Taalkundige bijdragen 1,52=awfr. *kreunia (aus *krevia). Die belege geua, iewa etc. (s. unten zu geva), neuen, newa $\mathrm{W}$, Sch 377. J 50,44, euen, ewen $\mathrm{W}, \mathrm{S}, \mathrm{J}$ 2, 31. 50, 41, eenen [even] J 58, 3 geben keinen aufschluss (nwfi. $n \hat{e}^{a} v e n$, pl. nếa ven).

Ein in ähnlicher weise durch nebenformen mit $\hat{\imath}$ vor abergang in $i \hat{u}$ geschutztes iu erblicke ich in lyun(e)ria, -wrien 'liefern' Sch 546. 617. 653.673. Ag 137. 146 (das $w$ aus *livr $\hat{o} s(t)$, $-\breve{o} t h,-\breve{o} d$ ) und zwar mit rücksicht auf das fehlen von flexionsoder verwanten formen, welche die erbaltung eines $i \hat{u}$ vor $w$ hätten veranlassen können; vgl. ubrigens liewerien Sch 537 und nwfr. lieuwerje GJ mit ieu als schreibung für iu. Daneben lyw(e)ria, -ien J 12, 34. 32, 3. 4. 5. 6. 46, 46. 62, 6. Sch 613. 695. 744. $\mathrm{Ag} 84$, lyurian Sch 663. 668. Ag 66 mit $y w, y u=\hat{\imath}-v$ oder $\{u-w$ ?

Unbestimmbar ist natürlich auch der lautwert der nach art von breue, geua etc. mit einfachem vocal $+u, v$ oder $w$ geschriebenen belege, insofern fur dieselben erhaltung des $v$ in den flexionsformen mit $-a$ oder hellem vocal in der endung oder durch anlehnung an eine form mit $f$ wahrscheinlich oder fur denkbar zu erachten ist: howes, -e hoves, -e, houe 'bofes',

J 21, 35, hieten p. p. J 20,1. 84.19. 24. 87, 3, lieten ger. und p. p. Sch 647. Ag 102, liete opt. praes. H 302. 303, grieta 'anklagen' H 108. 109. 139, grietinan Sch 742. W 105, 21. H 155. 160. 161, kriept 'kriccht' H 162, liesa 'lösen' H 43. 81. 170, haudliesne H 137. 138. 139. 149, giet 'geht' J 21, 17. 81, 13, hyede(n) 'hatte(n), hätte' J 1, 14. 20,4. 16,61. 63, 9. 70, 3, onmietlick J 9, 5, lyengued [lyengueed] J 20,1, died 'tat' Sch 601, Iwier 'zwei' Sch 600, ghien 'kein' Sch 661, etc.

1) Im Mnd. wb. wird die bedentung unrichtig als 'schaudern' angegeben. 
-e W 53, 15. 396, 12. 17. 401, 28. 413, 15. 30. 420, 19. 431, 34 . $\mathrm{H}$ passim. J 75, 4, howet, -al(h), -ad W 413, 2. 4. 417,33. S 384, 11. H 130. 139, loue 'lobe' W 431, 9. H 69.169, lowiad, lowath, -et, -ade W 75, 26. 426, 7. H 95. J 22, 7. 8. 23, 5. 84, 21, bilowalh $\mathrm{Ag} 99$, bilowad W 5, 5, lowinghe J 24, 4 ( $\mathrm{vgl}$. oben s. 352), lawa nachlass mit lanigia, -et etc. $\mathrm{W}, \mathrm{H}$ und $\mathrm{J}$ passim, lavie, -ede $\mathrm{W} 105,13.398,37$, (bi)rawia, -et etc. W, S, H und $\mathrm{J}$ passim, rawis, -e, raue $\mathrm{W}, \mathrm{H} 160.300$, rawer $\mathrm{J} 71,5, \mathrm{~S}$, lawa 'glaube' $\mathrm{W}, \mathrm{J}$ passim, rawerie $\mathrm{J} 60,1$, bliwa, -en $\mathrm{W} 428,36$. 432, 2. 35 . $\mathrm{H}$ 164. 172. 178. J passim, driva, -en, $-e \mathrm{~W}, \mathrm{H} 116$. J passim, scrina, -en, -e W, H 145. 162. 177. J 3,6. 5, 5. 13, 41. 15, 27. 18, 16. 26, 4, biclywen, cliwa 'bekleiben, wachsen' H 24 (vgl. nwfi. kliâne 'klimmen'), liwes, -e, liues, -e W, S, H 84. 94. 95. 114. 159. J 46, 47.66. 61, 2. 84, 8. 21, ontlyuet J 56, 9, liwet (h) 'lebt, gelebt' W, J 44, 2. 50, 40. 44. 73, 1, liwade 'lebte' W, J II s. 138 , etc. = houwe etc. oder hove etc., bliuwa etc. oder blîva etc. oder etwa nach art der formen mit eu statt $i \hat{u}$, iou blíuwa etc.? Vgl. wegen erhaltung des $v$ nwfr. hoaven pl. und lijvleaz 'leblos' GJ. Neben wives $\mathrm{W}$, wiues $\mathrm{H} 94$. J 85, 5, wiue $\mathrm{W}, \mathrm{H}$ 34. 83. 103. 104. 117. J 84, 23, wivet J 50,40, behôwet J 15, 5 (denom. zu bihôf $\mathrm{W}, \mathrm{S}$ ), have, haue $\mathrm{W}, \mathrm{J}$ 1, 23. 43, 2. 59,18, grewa (dat. sg. zu gref 'grab') H 47 habe ich keinen beleg mit iu etc. verzeichnet; vgl. auch nwfi. (GJ) wiven pl., behoafje, have und beachte noch nwfr. kîvje 'streiten', oeffenje 'üben' (aus *ôfnia, nicht $=$ aofr. ôvonia).

Zum schluss sei noch auf das lautverhältnis in lyauwe 'liebe' (adj.) Sch 591. 733, liauwera 'lieber' H 160 (und liawera? W 426, 37. 439,13), lyan uxor Ag 52.105. Sch 608, lyauuen 'deu hof machend' J 58, 26 1) (nwfr. ljeauwe 'geliebte', ljeauwert 'geliebter' neben ljeave, ljeavert GJ), tyaunes 'diebes' H 294 bingewiesen. Der zweite component des dem iâ zu grunde liegenden $i a<i_{0}$ und das $-a<-0$ sind die resultate ein

1) Part. praes. zu "liaunia $=$ ahd. liupôn. Die stelle lautet hwanneer een man een ôrem lyauuen menscha (lies menscha lyauuen)

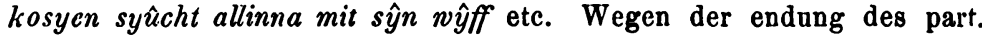
praes. -en für -ien vgl. bidauen 'betanend' H 149 (zu bidauwia $=\mathrm{nwfr}$. daunje, ahd. touuôn), bitellen $\mathrm{J} 7,2$, bychten $\mathrm{J} 15,15$ gerund., trouve $\mathrm{J} 84,3$ opt. praes., behowet $\mathrm{J} 32,24$ als 3 . pl. mit synkope des $-i$ - nach dem wuster -(i)gen, -(i)ge, -(i)get für -(i)gien etc. (s. unten zu hâna). 
und desselben entwickelungsprincips, nämlich der qualitativen schwächung bei tonloser aussprache. Weil demnach ia, gesckweige denn $i a \hat{a}$, schwerlich älteren datums als -o sein kann, muss altes $\{0-v$ zur zeit der genesis von $w$ aus $v$ zu io- $n$ zu iou- $n$ geworden sein und ist das liberlieferte iau (triphthong iáu) als die hieraus durch anlehnung an iâ von liaef, $t(h)$ iaef entwickelte lautverbindung $\mathrm{zu}$ fassen. Durch fortgesetzte anlehnung an $l i \hat{e}^{a} f$, tiế $\hat{e}^{a} f$ mit jungerem $i \hat{e}^{a}$ für $i \hat{a}$ (vgl. unten zu sauwen) entstanden dann in der folge das bei Wassenbergh 1, 161 belegte $\mathrm{nwfr}$. (Zuidhoeksche) leeuwe caros (d. h. léane mit schwund des ersten componenten, vgl. oben s. 351 anm.; in derselben quelle aber noch lauwer potius 157.159.168) und die ebenfalls bei Wassenb. 1, 144 begegnenden nwfr. (Molkwerumschen) leceun 'lieb', tjeceuven 'diebe' (d. h. le'a u, tjèa umen).

Bylyowa 'gewähren'. S. oben s. 356 .

Binaemd. Das part. praet. begegnet in verbindung mit moerd und moerdbrand in $\mathrm{J} \mathrm{1,33}$ Hweerso een man jefta een wŷf bârnd is an der wallenda wêge [wêghe] om een bynaemd moerd jefta moerdbrând, soe schel met ferst (man die frist) wŷsa wrnacht ende ita lettera (im folgenden) eetmeel dyn hals wrordêlya. Ebenso nômad, noemed in $\mathrm{H} 111$ Hweerso ên $n \hat{y} f$ iefta man bârnd is in dat wâllende wetter om ên nômadne moerdbrânt iefter noemed moerd, soe schel (lies schel me) ôrdelferste wîsa dus (lies duers ${ }^{1}$ )) wrnacht ende etta lettera etmêle den hals wrôrdelia. Was die ausdrucke bezeichnen, geht hervor aus $\mathrm{W}$ 412, 17-20 (und H 136 mit für unsern zweck unwichtigen va-

1) Vgl. dwers (dwars) wr nacht J 2,31. Sch 540.671, t(h)wers wr nacht Sch 547.601 = 'unmittelbar am andern tage'. Behufs erklärung der bedeutung sei hingewiesen einerseits auf aofr. thweres $\hat{a} r=$ "bis zul" andren seite hintiber' (d.h. 'in der breite') in meta ondling (in der länge) and thweres $0 r \mathrm{~B} 178,27$, andrerseits auf $w r$, $\hat{r} r=$ 'ultra' (in folge von ibertragung vom antecedens auf das sequens, aus 'trans') in $\hat{u} r$ nacht, $\hat{u} r$ achte dagen etc. $\mathrm{W}$; also dwers, twers $w r$ nacht $=$ 'unmittelbar nach ende der nacht'. Mit mhd. über twere, querde naht, mnd. over dwernacht, mnl. over dwersnacht stimmt unser ausdruck mithin was das resultat der begriffsentwickelung angeht iiberein, nicht aber in der entwickelung selber: twere etc. nacht $=$ 'nox transversa, die die beiden tage quer durchschneidende nacht'; über twere nacht etc. = 'ultra noctem transversam'. Die form mit $d w$ neben der mit $t w$ (thw residuum der alten orthographie) scheint auf eine dialektische durch $u$ bewirkte 
rianten), wo es in einer beschreibung der bei einer mordklage üblichen processordnung heisst $N u$ di eerfnama di oenbringh deel (lies deeld) is (nachdem vom aesga auf geheiss des grewa dem klagenden erben das recht, seine klage zu beschwören, zugeteilt ist), nu schil hy swara op dae helligem ende naemt dine man, deer deer slayn is. Binaemd, nômad (moerd) brând ist also ein mord(brand), dessen opfer namentlich genannt wird.

Mit on(bi)naemd, unnamed, o(e)nnaemd, onenaemd moerd muss demnach ein mord gemeint sein, dessen opfer nicht namentlich angezeigt werden kann. Und so finden sich diese ausdricke öfters in unsern rechtsquellen angewendet in bezug auf eine verletzung, welche einen mann oder eine frau der zeugungsfähigkeit beraubt hat, demnach als indirecter mord der kinder, die sonst eventuell hätten gezeugt werden können und folglich nicht als opfer nachzuweisen sind, gelten durfte. S. auch v. R. i. v. unnamed und beachte $\mathrm{S} 447,24 \mathrm{ff}$. (= H 207, § 41) Hweersoe thi man schetten ward truch sine machta truch thet fel, thiŏ bute is ên pund..., hit ne see thet hi fêrra wille spreka, soo mey hi habba thri $\vec{a}$ sinekerff (kann er dreierlei vergutung wegen zerschnittener sehne bekommen)... the arste

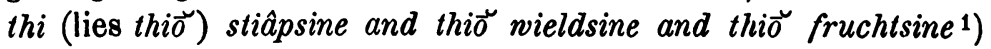
..., thêr môth hi fan tigia; thriă unnameda môrd âchma te bêlane also dyôre als an manslachta iefla tolvasum unswerra (sich freischwören zu zwölfen, d. h. mit elf eideshelfern); sowie W 470, $22 \mathrm{ff}$. (= H 239, § 27) Hueerso ên man truch sŷn machta schetten wirt truch dat fel, diö boet is II einsa, hit ne sê dat hi fôra sprecka wil, so mei hi habba tri $\vec{a}$ synkerf. . . diō aerste

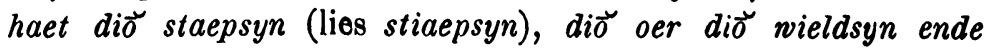
diŏ tredde diठ̆ fruchtsyn ..., deer moet hi fan bitigia; tri $\vec{a}$ onbinaemd moerd da aeghma allerlyc (jeden) to bêten so diôre so een manslachta iefta tolvasum onsvara; und $\mathrm{S} 456,32 \mathrm{ff} .(=\mathrm{H}$ 256, § 23).

Wegen andrer belege für diesen ausdruck s. noch W 403,

scbwächung des anlautenden $p \mathrm{zu} \delta$ hinzuweisen: s. auch dwingen $\mathrm{J} 23,6$. 43, 12 neben twinga, twong, -en W, H 22. 168. 171, J passim, twang subst. W, H 22. 145. J 12,13. 24,16, und nwfr. (GJ) dwerz, dwynge neben twos (aus *twors), ininge.

1) Was mit diesen drei sehnen gemeint ist, habe ich bis jetzt nicht ermitteln können. 
$21 \mathrm{ff}$. (= H 100, § 6): Jef ma een man wrôghet om een onbinaemd moerd, deer hi deen habbe oen da wîue (den er an der fiau verubt habe), ende hi des bisecka wil, so moet hy tolvasum onriûchta (sich freischwören), ief hy onschieldich is; und W 409, $7 \mathrm{ff}$. (= H 113, $\S 48^{1}$ )) Hweerso ma een man biclaget om een onnaemd moerd, so is hi niêr tolvasum to onswarane, dan hi aeg toe daiane sind iefla ôrdel iefta ênich [ênigh] oentiâgh. ${ }^{2}$ )

Aus diesen stellen ergibt sich also, dass der wegen eines on(bi)naemd moerd angeklagte sich mit elf eideshelfern freischwören konnte. Ein solches reinigungsnittel aber kam dem eines binaemd moerd verklagten nicht $\mathrm{zu}$; dieser konnte sich nur durch ein gottesurteil reinigen: Hweersoe ên man iefta ên whyf hiâra bârnd habbet in dere (1. da) wâllende wetthere om ên binaemd moerd iefta om ên binaemd moerdbrânde $\mathrm{H} 112$, $\S 42$ (an der parallelstelle W 408, § 15 steht onnaemd moerd, das naturlich als verderbte lesart für onbinaemd moerd $\mathrm{zu}$ fassen ist).

Biriind (?). Nach den aofr: stellen huasa thel godes hûs brecht and thêr binna tha hêlega berant $\mathrm{E}^{1} 30,12$, sa wa sa thet godes hûs brech and hi thêr binna tha helga berant Huns. 30,12 , und der awfr. dattet hollige corpus Domini britzen is, dy tzelck slort is ief di holliga funt stirt is ief britzen, dae holga birant $\mathrm{H} \mathrm{108,} \mathrm{mit} \mathrm{be-,} \mathrm{birant} \mathrm{als} \mathrm{3.} \mathrm{sg.} \mathrm{praes.} \mathrm{ind.} \mathrm{bez.} \mathrm{p.} \mathrm{p.}$ *be-, birenda 'zerbrechen', dtrfte man an der parallelstelle des letzteren citates ende dat hellighe corpus Domini britsen is, dy tzylick stirt is, dat funt britsen, da helligha birynd [biriind], W 406, 22, entweder birand oder bireind ev. birend erwarten. Vgl. einerseits ausser dem erwähnten birant die praeterita sance misit W 430,14, wanten verterunt $\mathrm{H} 169$ mit ursprlunglichem nicht umgelauteten vocal, andrerseits die praeterita seinte $\mathrm{W}$ 439, 24. $\mathrm{H} \mathrm{64}$, sente $\mathrm{H} 168$, weinten $\mathrm{W} 431,27$ und die p. $p$. toreind W 472,5, seint, seynd $\mathrm{H} 165$. J 15,42. 18, 15, saynt W 428, 25 (ay als schreibung fur ei, vgl. unten ayn(d), aynt, snaynd, toraint, sowie maytia, ainse, s. zu meit(b)ia und wrichte

1) In der überschrift des $\S$ steht falsch nômada moerd.

2) Ebenso im aofr. F 42 Hwêrsa ma ânne mon biclagat umbe ên unebinômat mord, sa is hi niâr XII sum to untswerane, than hi age to deyane sineth ieftha en ontiuch. 
'arbeiter') mit unurspr'tnglichem, aus den praesensformen seynden $\mathrm{W} 422,12 . \mathrm{J} 18,23$, seynda $\mathrm{J} 15,31$, seinde opt. W, H 65. 144, serda(ne) S, H 143, * weinda (die praesensformen sind nicht belegt) entnommenem wurzellaut. ${ }^{1}$ ) Dass hier in graphischer hinsicht eine ursprlingliche lesart bireind als die wahrscheinlicbste zu erachten ist, liegt auf der hand.

Wegen des $e i$ aus $e$, umlaut von $a$, vor $n d$, nt vgl. noch reynd 'das zerreissen der kleider' Sch 547. 599. 720, beynd 'band' H 113, heerbeinden $\mathrm{W}, \mathrm{J} 25,8.60,22$, eind(e), ein finis $\mathrm{W}, \mathrm{H}$ 139. 148. 158. 175. J 9, 2. 17, 6. 18, 2. 10. 11. 13. 16. 19, 5. 21,31. 24, 12. 46, 13. 47, 4, ayn(d) J 31, 2. 36, 10, sneynd(e), snaynd 'sonntag' (s. s. v.), eynda, -en(e) 'enden' W, S, H 73. J 2, 24. 21, 18, eynt 3. sg. praes. ind. und p. p. J 2,30. 3, 1. 9, 2. 15, 35. 18, 3. 11. 13. 16. 2।, 10, aynt J 15,35. 18, 10. 3।, 2, eynd(i)gie, -get etc. W 438, 31. H 29. J 15, 71. 19, 5, eynd-, eyntlick J 4, 1. 12, 18. 17, 6. 24, 12. 86, 3, eynlick J 18, 3, heynd 'nahe' J 20, 14. 50, 32 (ahd. gehente), peynda, -en 'pfänden' Sch 536. J 59,2, weinden (s. unten s. v.), in-, wtweyndich J 33, 1. 77, 8, tweintich W, H passim.

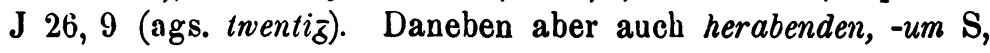
end(e) S, J 2, 30, enda subst. J 50,41, endena ger. Sch 394, endeghia $\mathrm{H} 150$, entlik(e) W, J 8,3.15,71, penda W, H 132, in-, wlvendelyck J 46, 19, wenden 'ausnahmen' W 32, 2. J 2, 31,

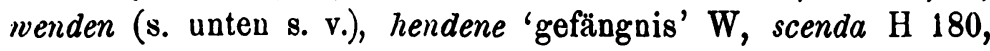
sente, senda(ne) (s. oben), sent und torenth (s. die fussn.), lenth (s. unten s. v.). An ein wirkliches $e$ neben $e i$ wäre hier selbstredend nicht zu denken; es sind vielmehr, auch mit rttcksicht auf das durchgehende ey im nwfr. (vgl. in GJ eyn 'ende', eyn 'ente', heyn 'nahe', seyne 'senden', weyne 'wenden', scheyne 'schänden'), die letzteren belege als residua der alten orthographie zu fassen, die eben deshalb ziemlich häufig verwant wurde, weil zur zeit unserer quellen die diphthongische qualität sich noch nicht völlig entwickelt hatte, d. h. die ei-aussprache noch nicht ganz deutlich gehört wurde.

$\mathrm{Zu}$ beachten ist ferner, dass die schwachbetonte silbe nicht ei hat: ellende W, H 24. 144, el(l)endich(eed) J 25, 29. 73,1, inlende

1) So auch die 3. sg. praes. ind. toeraint $\mathrm{W} 472,6$, torenth $\mathrm{S} 480,28$, seint W 404, 27. H 97. J 19,4. 47,6, sent W 402, 22, statt urspr. (toe)ranth $\mathrm{S} 498,31$. H 242 , sant $\mathrm{H} 102=$ aofr. rant, sant. 
'ackerland' H 136 (abd. gilenti, gilenda culta, arva), en(de) und: Vgl. nwfr. (GJ) illinde, -ig, in und en.

Bisetta 'einsprache gegen ein gerichtliches erkenntnis erlegen'. S. unten zu foerdwhsa. Im vorbeigehen sei hier das Beitr. 17, 306 unrichtig als 'besessen, verruckt' gefasste aofr. bisette erwähnt in Hwasa blend ief hha hôxnath ênne mon mith waldand and mith bis et te hei, sa bêtema him thribête B 159, 26. Das schwache part. kann nicht zu bisitta gehören und ausserdem wäre eine bedeutung 'besessen' hier nicht am platz. Man denke an mhd. sich selzen $\hat{u}$ etwas 'sich etwas fest vornehmen' (Lexer 2, 895), mnd. sik dârtô selten 'seinen willen darauf richten' (SchillerLubben 4, 199) und libersetze mith bisette hei mit ricksicht auf die häufige verwendung von be- in der bedeutung 'ad, in (c. acc.)' nach der Aofr. gr. s. 222 durch 'intento animo'.

Bescât 'bestimmt'. S. zu wrichte 'arbeiter'.

Bisplitta in J 71,5 Dat ma da monicken moet bysplitta ende byrawia, hwanneer hy $\vec{a}$ hyaere regula naet hâldeth ende mey ma foeriagia als raweren ende wolven. Hinsichtlich der nicht $\mathrm{zu}$ verkennenden bedeutung des verbums 'berauben' ist mnd. besplîten zu beachten in He heft unse underdanen ... geschinnet, bespleten unde gheschattet Friedländer, Ostfries. urkundenbuch no. 1441.1) Es vergleichen sich die ags. composita bebrecan, beceorfan, be-, bihéawan, besittan = 'einen oder etwas durch brechen, kerben, hauen, abreissen, von etwas berauben'2), (eig. 'das brechen etc. an einem oder' an etwas verrichten'). Bysplitta muss demnach urspr. im eigentlichen sinn 'durch reissen oder lostrennen berauben' geheissen haben und als solches in bezug auf bestimmte gegenstände verwant sein; die uberlieferte bedeutung kann nur als die folge von metaphorischer ubertragung gelten. ${ }^{3}$ )

1) Die stelle wurde mir freundlichst aus Lübbens handschriftlichem nachlass von herrn $d r$. Walther mitgeteilt.

2) S. die in Greins Wb. citierten stellen béam héo (yldo) ábréoted and bebrice o telzum; hine (hie) héafde becearf; hél Johannes... héafde behéawan; Jaet he Johannes bibéad héafde bihéawan; Jec sculon moldwyrmas céowan, seonowum beslitan; besliten seonwum.

8) Vgl. behéawan in hwonne mec wrádra sum aldre behéowe Gen, 2701. 
Die schwache form mit $t$ begegnet auch in mnl. nnl. splitten, engl. to split, dän. splitte gegenuber aofr. splita, mnd. nnd. splîten, mnl. spliten, nnl. splijten etc.

Blôya. S. zu grôya.

Dwers ûr nacht. S. oben zu binaemd.

Enka 'ackerknecht'. S. zu inka.

Ent(b)erâ 'einer von beiden' und 'entweder'. Das $\hat{e}$, gleichviel welcher herkunft, wird in awfr. vor $n n$ und $n+$ dental gekurzt und zwar zu einem vocal, der nach massgabe der wechselnden schreibung mit $i(y)$ und $e$ qualitativ zwischen $i$ und $e$ lag, also phonetisch durch $e^{i}$ zu bezeichnen wäre (ausnahmen wie seent 'suhnt', seend 'gesthnt', meent 'meint', bileende 'belehnte', meent 'gemeinde' etc. erklären sich von selber):

allinna 'allein' W, J 2, 9. 3,12. 13, 24. 27. 15, 15. 52. $21,5$. 25, 12. 30, 8. 16 etc. Sch 709 und allenna S, H 176; stins 'burg' J 26, 5. Sch 517. 726 (aus *stênisse mit suffix *isjô zu stên, wie ahd. burissa zu bora 'bohrer', ags. lynes 'wagenlünse' zu abd. lun; wegen der bedeutung vgl. mnl. steen 'burg'); hyndicheed, byhyndiget und henda (s. unten zu ersterem wort); nwfr. klinsgje 'reinigen' GJ mit constantem $i$, wie in nwfr. allinne, stinze $)=$ awfr. *klins(i)gia, *klens(i)gia (= ags. clénsian; wegen -(i)gia s. unten zu hana),

und ent(h)era 'einer von beiden, entweder', nent(h)era, nint( $h$ erd ' $k$ einer von beiden' in Hwannêr da iggen (parteien) sint commen jefta entherd fanda iggen $\mathrm{J} 2,12 ;$ Foer enthera igh fan da ôrem een pêna byjerrie mey, so ist need ende byhoeff, dat dy $\breve{o}$ pêna to fara fan da igghen êniget sê $\mathrm{J} 21,28$; man ende wŷff agen hydre caepland lŷck to dêlen, als hydra enterâ sterfı J 82, 17; mey ma dat hellighe aefı schêda ... joff hiâra [hidre] entherâ wil to conveynt $\mathrm{J} 85,1 ;$ jef enterâ paert (partei) jef hierra tzirlen van oerem hynxlen ... heed Sch 706; alsoe lang als entrâ fan mŷn twâm dochtren forsz. lyffheftich synt Sch 724; dat hi him scel entrâ kestigia iefla opthinghia $\mathrm{H}$ 133; dat hi aldus grët (angeklagt) anderdia scel entra ti iechtwerde iefta ti seckwerde $\mathrm{H} 135$ (ähnlich $\mathrm{H}$ 136. 137); entrâ deis iefta nachtes $\mathrm{H} 163$; nenter a eegh (partei) oeren wielt to dwaen Sch 706; deer nenterâ eegh ôrem yn thoe maestrien Sch 706; so schil nenterâ paert oerem mâsterie Sch 706; enne nentrâ oerm meer wyeld 
to dwaen Sch 609; Hwanneer datter twee [tweer] man habbet schylinghe ... ende hy $\vec{a}$ swîget $X$ jeer langh ..., deer ney mogen hy $\vec{a}$ naet sprecka nenther $\hat{a}$ op ôrem ...., ende hy $\vec{a}$ swîget dan $X X$ jeer ende spreckket nenterâ op ôrem, so moghen hy $\widetilde{a}$ nenter $\hat{a}$ eefflerdâm op ôrem sprecka J 36, 9; Hweerso deer twâa kŷnden to gâra joun (gegeben) wirdeth bynna saun jêrem ende schêdese dan in da saunde [saunda] ieer, so moet ayder in ôderis slacht boestigia (heiraten), sint se to gâra wr da saun jeer, so moet nenther $\hat{a}$ in ôders sib faen $\mathrm{J} 84,15$; Jeff een man op eenre stoe tnâm frounen trouna louwed, so môther nent her $\hat{a}$ nymma J 84, 27; hwant hâlt nintera (keine der beiden parteien) fan nên pêna, so moghen da soenlyoed deer nên bij lidze J 21, 25.

Enter in ent(h)erâ entspricht mhd. eintweder aus ein und ahd. deweder, welch letztere bildungen sich durch zweierlei eigentlimlichkeit, den schwund des $h$ von deh- und das $t$ aus $d$, kennzeichnen: altes *thehhwedhar hätte bei ungestörter entwickelung historisches dehhweder ergeben, nicht deweder, das auf ein durch anlehnung an *hwedhar für * thehhwedhar eingetretenes * thehwedhar zurückgehen muss; durch die synkope des unbetonten vocals in -de- aber kam der dental des durch anlehnung an weder in einde-weder abgeteilten compositums in den silbenauslaut des ersten compositionsteils zu stehen, wurde mithin zur fortis. Der erstere vorgang ist auch für den schwund des gutturalen spiranten im awfr. geltend zu machen. Die genesis des $t$ gestaltet sich hier jedoch in anderer weise. Die verbindung des ên mit der pronominalen form fand entweder statt, als das eine schwachtonige silbe anlautende th schon der regel gemäss stimmhaft geworden war (vgl. awfr. $d i$, $d a$ pron. und artikel, doch, dana, $d \vec{a}$ ' $\mathrm{da}$ ', dus, dan, $d u$ pron. pers. mit $d$ aus $\delta$ in unbetonter silbe), oder vorber. Im ersteren falle hätte *êndeweder ${ }^{1}$ ) entstehen müssen mit $d$ für stimmhaftes an vorangehendes $n$ angelehntes th (vgl. Aofr. gr. $\$ 125 \gamma$ ) $>$ zwar zunächst nach svnkope des vocals $*_{\vec{e}}$ ntweder mit $t$ als fortis, in der folge aber nach der synkope des $w$ (vgl. unten zu inka) mit herstellung der fruheren qualität des nun in den anlaut tretenden dentals *ender (vgl. a. a. 0. anderk, anderdia etc.

1) Ueber fries hweder mit $d$ gegenüber $p, \gamma$ in got. hwapar, as. hweðar etc. vgl. Aofr. gramm. s. 97. 
gegenliber andwirk, andwert etc. mit als fortis gesprochenem d). Bei einer verbindung des ên und *theneder (mit stimmlosem $t h$ ) bätte sich bingegen zu der zeit wo die spirantische qualität der sogenannten interdentalen verloren giong, eine form mit festem $t$ entwickeln müssen. (Die aus den oben angefuhrten belegen zu ersehende bäufige schreibung th für $t$ beruht auf reminiscenz an die alte orthographie, vgl. unten zu meyt(b)ia). Wegen des schwunds von $d$ durch assimilierung mit $r$ vgl. hoer, hôr utrum $\mathrm{W}, \mathrm{S}$ aus hoder W, S, roer 'ruder' W, H 64 aus rôder W, weer(-) 'wider(-), wieder(-)' W, H und J passim aus weder(-) $\mathrm{W}, \mathrm{H}$ und $\mathrm{J}$ passim, onmeer 'unwetter' $\mathrm{W}$ aus onweder $\mathrm{H} 83$, broer 'bruder' $\mathrm{W}, \mathrm{H} 33.48$. 159. J 15, 27. 30, 18. 38,63. 46, 24 aus brôder $\mathrm{W}, \mathrm{H}$ und $\mathrm{J}$ passin, moer 'mutter' W 389, 23. J 22,19. 26, 17. 47, 10. 57, 3. 4 aus moeder $\mathrm{W}, \mathrm{H}$ und $\mathrm{J}$ passim, faer 'vater' $\mathrm{J} 47,10.57,4$ aus fader $\mathrm{W}$, H und J passim, to gâra, (to)gaer W, H 63. 113. 118. 140.166. 179. J passim mit gâria, -ien etc. W 463, 24. 461, 4. 436,6. J 2, 28. $27,1.46,45.83,3$, wrgâringhe $\mathrm{J} 3,1$ aus to gadera, (to)gader $\mathrm{W}, \mathrm{H} 159.176$ mit gad(e)ria, -et W 409, 18. 431,3. H 112, oer uberall passim aus ôder uberall passim, etc. Wegen des an das indefinitum angehängten $-\hat{a}$ 'immer' $s$. unten $\mathrm{zu}$ hat $\mathrm{a}$.

An zwei stellen wird ninter $\hat{a}$ auch in bezug auf mehrere personen verwant: nu schil di brôder habba een pond (als anteil von der meenteel), ief hi deer is, ende ief hi deer naet is, so aegh hit to nimen sînes brôders soen iefta sînes susters soen, ief hiă deer ninterâ sint, so nima hit di eerfnama self $\mathrm{W} 410$, $23 \mathrm{ff}$; da natuerlike sonen schillet faen to da sexte deel dis gueden [guedes], jelle [jetta] datter (auch wenn da) aeffle sonen sê jeff fader jefta môder off nŷff wêre; haeth hij (der vater der unehelichen kinder) dera nynther $a$ libben (am leben), so moet dij fader da natuerlike sonen ... opjà al sŷn gued J 47,8. Im nwfr. (GJ) gilt ninter ausschliesslich für 'niemand, kein' (auch nintertijd, nintertijds 'zu keiner zeit, nie', wonach als neubildung intertijd ' $\mathrm{je}$ ').

Fatiender (mit-bernte), fatiande (mitter-berdene) in ief hi (der dieb) an dae flechtiga foet ende mit fatiender bernte biginsen (ertappt) virt $\mathrm{W}$, und an der parallelstelle in $\mathrm{H} 162 \mathrm{Jef}$ hi an dae flechtiga foete ende mitter fatiande berdene beghenzien wirt. Das part. gehört zu einem dem ahd. 
fazzôn, mhd. fazzen 'packen, aufladen' entsprechenden *fatia; seine verbindung mit dem subst. bernte, berdene 'pack, last' vergleicht sich in syntaktischer hinsicht mit dem part. in ênen stouwenden eeth $\mathrm{S} 482,15$, in aofi. dregande clâthera $\mathrm{R}^{2} 540,8$, drîwant and dregand gôd $\mathrm{B} 165,4$, drîwende iefte dregande gîd $\mathrm{E} 2197$, 24, in mnl. vallende sieckte, singende mis, wevende werken, in nnl. op komenden wege, wandelende boterham (ein butterbrot, das man im hin und her gehen verspeist), loopend werk, zittend werk (arbeit, die man laufend, sitzend verrichtet), ijlende koorls (fieber, in dem man phantasiert) etc., und in den von Grimm, Gramm. 4,65 erwähnten mhd. ausdricken ansehende leit, windende hende, lebendez leben etc. (die von der person ausgehende tätigkeit wird auf die sache übertragen, an welcher die tätigkeit zu tage tritt).

Dasselbe verbum begegnet auch im aofr. und zwar als p. p. in end ma hine (den dieb) befereth . . ar ther fat ada bernde Huns. 36, 16 (s. Beitr. 14, 263 anm. 1; das Beitr. 17,310 verzeichnete fakia ist demnach zu streichen).

Die nwfr. form von *fatia lautet fettje GJ, das auf analogischem wege nach dem muster von hellia, berria, meckia etc. neben halia, baria, makia etc. (s. oben zu bihellet) gebildet sein muss; denn von dem in den letzteren formen zu beobachtenden awfr. lautprocess findet sich vor $t$ keine spur; vgl. batya J 17, 15. 26, 13. 58, 24, hatia (8. unten 8. v.). Ueber ein auf gleichem wege entstandenes wannia s. zu wennia.

Ferthe, firde 'geleite'. S. zu wta.

Fíand. Zur regel ' $i-a$ und $\hat{\imath}-a$ zu $i a$ zu $i \hat{a}$ bez. $i \hat{e}^{a}$ ' bildet der awfr. reflex von got. fijands, as. fîand etc. (s. Beitr. 15, 467 f.) durch einwirkung der nomina auf -and, nämlich warand, warend, werend 'burge' W 53,10. 398, 10. H 56. 86 , berand, -end 'butge' $\mathrm{B}$ 43. 44. 45. 130 und vorztiglich *nigand = aofr. wigand, eine ausnahme; denn dass fiand $\mathrm{W}, \mathrm{H} 71$. 83. 117. 159. 170. 178. 179. J 5, 4. 6, 2. 8, 5. 56, 1 als fîand und nicht als fând anzusetzen ist, geht hervor: primo aus der constanten schreibung des wortes mit $i a$ (nie iae); secundo aus dem neben dieser form begegnenden, durch schwächung des endungsvocals entwickelten fŷnd $\mathrm{W} 7,4$. S, H 300. J 27, 1. 55, 4. 59, 18. 19. 65, 2, 71, 6 (nwfr. fŷnn GJ). 
Fyûchtleeck in Hweerso een dobbelspil is in een huus, haet so dŷr schŷd fan jeld to lênen jeffta fan fyâchtleeck, al wôrde een menscha slayn off môyed, bêda dij huushêra ende aeck dij dobler dâ mogen in neen rîcht sprecka om bettringhe off om leend jeld $\mathrm{J} 28,21$. Das wort ist zusammengesetzt aus */iuchta (= aofr. fâchte, ags. feoht) und *lêk (= ags. lác, an. leikr 'kampf(spiel)') und entspricht mittelniederländischem vechtelic 'gefecht'), ther dessen (auch im mnl. hu(ve)lic, hilic 'heirat' $=$ ahd. hîleih und mnl. feestelic 'fest' 2) begegnendes) für -leec eingetretenes -lic man Tijdschr. v. nederl. letterk. 13, $214 \mathrm{ff}$. nachsehe.

Flet, flêmt, flait. W 413,4 so hwa soe sŷn [siin] (des ferdloes erklärten mörders) gued flet iefta flent, haset ende howat dei ende nacht, soe aegh hi dis koninges ban toe bêlane mit twâm pondem. Die erste der alliterierenden formeln kann nur eine bezeichnung sein für 'fortschafft, zur seite schafft (mit der betrügerischen absicht, das confiscierte gut dem fiscus vorzuenthalten)'. Demnach muss hier das von $\mathbf{v} . \mathbf{R}$ für das zweite verbum herangezogene an. flenna 'spannen' aus dem spiel bleiben. In der incunabel steht flêt (Germania 35, 18), womit natürlich auch flêmt gemeint sein kann, $d . h$. eine 3. sg. praes. ind. zu flêma 'fluchten' = ags. (ze)flyman, fléman fugare, mit $\dot{y}^{\prime}, \dot{e}$ aus $\dot{e} a$, wie aus fléam fuga hervorgeht. Das andre wort begreift sich als 3 . sg. praes. ind. zu fletta $=$ an. flytja 'fortbringen, -tragen'.

An der parallelstelle von $\mathrm{W}$ steht in $\mathrm{H} 139$ flat iefta flait statt flet iefta flêmt. Fur die zweite form ist an mhd. vlougen, vlôgen fugare zu denken, das auf altes *flougên oder -òn zuruckgeht. Dem einen sowol wie dem andern wüde awfr. *flagia entsprechen (die schwachen verba 3 . klasse mit langer wurzelsilbe sind im fries. allesammt in die 2. uber-

1) S. ghescal, vechtelic of manslachte Sch 60. r. (wegen dieser and der folgenden abbreviaturen $\mathrm{vgl}$. meine Middelned. gramm. $11 \mathrm{ff}$.); soe ghesciede in der stede van delf alsoe groten vechtelic... soe datter omtrent $X X X V I$ man verslaghen worden $\mathrm{Gkr} .81 \mathrm{r}$.; sowie Gkr. $76 \mathrm{v}$. BE. 208 v. Dcr. 83 r. Cr. 62 v. 68 r., Rechtsboek van Utrecht 1, 9, 27.

$\left.{ }^{2}\right)$ In dattet volc van romen feestelic hadden opten eersten dach van oest Passionael Winterstuck 106 v. 
getreten) oder aber mit $j$ aus $g$ vor $i$ und diphthong aus dem wurzelvocal + durch $j$ erzeugtem $i$ (s. oben s. 347, wie in biclaia H 178. J ms. s. 23, opclayen $\mathbf{J} 62,13$ für (bi)clagia, -ien W, S, Sch 394. J passim, und daia, -iane, -ien, -ie 'dulden' W, S, H 108. 109. 111. 113. Sch 546. 609. 717. J 38,4. 45, $10=$ aofr. daia, Gramm. $\S 288 \beta^{1}$ ), für *dâgia $(n)$ etc.) *flaia, zu dem als 3. sg. praes. ind. ein für regelrechtes *flagath oder -eth eingetretenes *flaiat $(h)$ oder -et $(h)$ gehören konnte, das bei schwund des endungsvocals nach $j$ flait ergab ( $\nabla g l$. wegen dieser synkope layde praet. W, J 3, 4. 8, 7 und layd p. p. 3, 5. 8, 4. 15, 30. 18,9. 31,3. 11 etc. für *layede und layed, -eth J 3,4. 5,5. 15,37 , die ihr $j$ für $d$ aus den praesensformen laya, -ye etc. $\mathrm{W}, \mathrm{H}$ 160. J 3,1.6. 9. 13,11. 15, $30<$ ladia, -ie etc. W, $\mathrm{H}$ passim ${ }^{2}$ ) entnahmen).

In flat aber ist wol nur eine durch das folgende flait veranlasste verschreibung für flet zu erblicken.

Flyûscâper, fliuesdên caep. J 32, 22 Dij caep, dŷr mecket is op wirderinghe [verderinghe] ende baringhe (der abgeschlossen ist unter der bedingung einer gerichtlichen taxierung und preisbestimmung) ${ }^{3}$ ) ende da baerlyoed ${ }^{3}$ ) habbet naet barrel ${ }^{3}$ ),

1) Auf grund der fitir das aofr. statuierten entwickelung eines diphthongs ans vocal vor $w$ (s. unten s. 376 anm. 1) ist für diese dialekte die analoge erscheinung bei vocal vor $j$ anzuerkennen; also aofr. daia und beya, deya, ervemeithe etc. für die in der Aofr. gr. $\$ 143$ und sonst angesetzten daia, bêya, dêya, ervemêithe etc.

2) Durch ïbergang des $d$ in $j$ vor oder nach hellem vocal: schaia, -ien etc. W, J passim aus schadia, -ien etc. W, J 12, 4. 6. 7. 17. 18, 21. 32 etc., bireya 'iiberfuhren' W 425, 27. H 158 aus *biredia (Beitr. 14, 266), snîa 'schneiden' W, J 46, 17 aus snîda, -en J 58, 41. W, H 112, lya, -en 'leiden' J 20, 14. 22, 19. 30. 17. 58, 8. 62,16. 63, 2. 9. 64. 3. 86, 10 aus $l \hat{y} d a$ J 44, 1. 58,41. 82, 12, foer-, wrmîa 'vermeiden' $J$ 72, 7. 59, 18. 84, 5 aus *foer-, wrmîda, deya 'töten' W, H 64. J 24, 17. 58, 8. 25. 3i. 72, 8 aus *dêda (got. dau pjan), bl $\hat{y}$ 'froh' $\mathrm{H} 68$ aus *blìde (as. blî̀i), binia, binnia 'unter, unten' W 389, 20. 399, 13. 35. 463, 7. 465, 11. 467, 8. 27. 476,14. H 300 aus bi-, benida H 33. 59. 60. 112 (nida W 409,17), mei 'mit' W. H und J passim aus mede H 303. J 17, 1.21, 34. $45,1.85,3.87$, 1, etc. etc.

s) Die baerlyoed oder -liôde, welche in der regel in den gerichtsacten als die einen vergleich erwirkenden schiedsmänner erwähnt werden, daher anch wol soenlioed heissen (vgl. die mehrzahl der in v. R.'s Wb. i. v. bare aus Sch citierten stellen), erscheinen mitunter auch als die 
dij caep is van nêner werden ende is naet ende mey neen caep hêta ende is een gelikenisse fan een caep, men neen weerafflich caep; deer seyt dat riâcht van, dat aldulke caep is gelikenisse fan een caep of een fliuesdên caep ende is fan rî̀cht neen caop nêr aegh neen stal to habben foer riucht, als to fara [fare] wal benŷsd is. Die bedeutung dieses fliuesdên caep 'nicht rechtskräftiger kauf' liegt auf der hand. Fur die deutung des ausdruckes ist das in demselben paragraphen folgende $\mathrm{zu}$ beachten: Ende om dissen willa so is dij caper al da fruchten schyldich to recknien in dat bital (den ganzen feldertrag in rechnung $\mathrm{zu}$ bringen bei dem kaufpreis, d. b. eine verglitung fur den ganzen feldertrag ausser dem kaufpreis zu entrichten), deer (den) dij câper wtjown (bezahlt) haet, alst wal wtwŷst dat capitel ad marg., dŷr seyt: Hwaso is câpien lânden, deer hym foer jeld seth (als unterpfand verliehen) sint, op worderinghe [werderinghe] ende dat ayn alzo (d. b. obne werderinghe) bycrîgia wold, dat is een flyâsdên caep ende aegh neen stal, ende dij câper is schyldich dat lând neer to kêren ende da fruchten off renthen schel dij câper offslaen oen (ablassen von) dat jeld, dŷr hy deer op jown (gegeben) haet, omdat hij dat lând haet brûckt, eer dat hyt bitellet ende werdereth was; deerom is hij een flyâscâper ende is dat lând mit da fruchten schyldich weer to kêren.

Nach mnd. vlûs = 'flies' (vgl. ags. fly's, mhd. vlius vellus) und metaphorisch = 'feldertrag' (Lubben-Schiller) ist ein awfr. $f l i u(e) s=$ 'feldertrag' anzusetzen. Flyâscâper heisst also eigentlich 'käufer des feldertrayes', speciell 'der käufer eines landes, der wegen vernachlässigung der stipulierten rechtsformalitäten gerichtlich nicht als solcher, sondern nur als käufer des von ihm während des besitzes aus dem felde bezogenen ertrags gilt', also 'ein die stipulierteu formalitäten nicht beobachtender

gerichtsbeamten, denen bei abschluss eines kanfs die bestimmung des kaufpreises oblicgt; vgl. Ick Sŷnert hlîe ende bykanne... hoe dat ick Hasscha convente hab vercacpet... mîn huus ende hoef ende al mîn land... om een summa gildes also graet, als dat set ende barret is van dae baerliôde, deer (die) deer wr (bei) ende oen wessen habbat Sch 695. Baer, für *bare, das 'gutachten' dieser beamten (der schiedsgerichtliche oder den kaufpreis bestimmende spruch) ist derivatum zu altem *bar $=$ as. bar 'offenbar'. 
käufer'. Letztere bedeutung konnte die entwickelung eines flyâs- = 'ohne beachtung der rechtsformalitäten' hervorrufen und so die bildung eines andern compositums fliuesdên $=$ 'ohne beachtung der formalitäten abgeschlossen' veranlassen.

Fliu (e)swerp. Wie vor $w$ aus spirant. $v(t)$ (s. oben zu biliouwa causativ), erlitt der vocal vor ursprunglichem halbvocal $w$ diphthongierung ${ }^{1}$ ):

blaun Sch 377.615 (an letzter stelle fehlerbaft blauwe) mit $w$ aus den casus obliqui - clau 'hacke' H 122 (ahd. chlawa 'klaue'; vgl. wegen ersterer bedeutung das bei Kiliaen verzeichnete klauwe rastrum) - pa(e)unes, -is $\mathrm{H}$ 68. 106. 145. 159. 160 (as. pâvos), paues $\mathrm{W} 423,26$, pa(e)us $\mathrm{W} 406,5.407,1$. 408, 10. 23. 410 , 9. 425,10 . 440,16 . 441 , 4. 12. 32. H 68. 108 . 111. 158. 159. 160. 163. 170. 195. J 1, 37. 2, 32. 3, 6. 18, 12 . 20, 2. 21,36 . 23, 11. 24, 2. 27, 1. $28,14.71,1.81,14$ etc. $\operatorname{taun}(e)$ 'ausrlistung, geräte' 2) (ahd. gizâwa supellex, ags. zetáwe 'rtistung') - nauwer neuter H 48 (2 mal, = aofr. nôhwedder, Gramm. § 259) und iauwelik quisque (= aofr. iâhwelik, s. zu sauwen), die beide fur die chronologie der lauterscheinung auf die nach dem ausfall des $h$ liegende periode hinweisen froun (e), frou $\mathrm{W} 400,9$. 409, 13. 429, 2. 470, 8. H 2. 3. 32. 113. 115. 117. 149. 159. 165. 166. 171 ete. J 37, 14. 81, 15. 84, 12. 20. 25. 26. 85, 2. 3. 86, 2. 5. 8. 87, 2.7 etc. (aus *frôwa, s. Beitr. $16,310)$;

craul- 'haken' in craulcruum (l. craulcrum) W 469, 4 (ahd. crouuil, Beitr. 14, 233) - dau ros H 149, bidauen 'betauend' ib. - thaues in thaueswîsa (s. unten zu nâtb) und $t(h)$ aulic in $t$ (h)aulic riucht 'gewohnheitsrecht' W 434, 25. 435, 20. H 20.21 und $t(h) a u l i c$ pliga 'gewohnheitsmässiger brauch' W 435, 10.

1) Vgl. über die nämliche lauterscheinung im aofr. Gramm. $\S 38 \gamma$, wo jedoch unrichtig auwande, nâuvet etc. statt auwande, naunet etc. angesetzt sind.

$\left.{ }^{2}\right)$ In ên schep [schip]... sonder allerhânda rêmen ende rour ende taune $\mathrm{H} \mathrm{64}$, und fisktau(v) 'fischgeräte' Sch 662. 743. Vgl. auch aofr. tauvon (d. pl.) 'geräten' $R$. Das wort ist nicht $\mathrm{zu}$ verwechseln mit dem ntr. mnd. mnl. touwe 'geräte' < "tôwi (formell = got. taui; wegen ou-w aus $\hat{o}-w$ s. Beitr. 16, $306 \mathrm{ff}$.), mhd. gezouwe (mit analogisch entwickeltem $o u-w<\hat{o}-w j$, 8. Beitr. 17, 564 f.). Das mhd. fem. gezouwe 'geräte' verdankt sein ou für das bei regelrechter genesis zu erwartende $\hat{a}$ ( $\nabla g l$. ahd. gizawa) der anlehnung an zounen. 
H 21 (ags. ঠéan, as. thau, ahd. kathau disciplina, dauth moralis) - houweth 'baut' S 348, 19, houwen p. p. H 238. S 497, 38, hauven p. p. S 448, 33 - scouwia, -ien 'schauen' H 104. S 448, 21. 496, 29. Sch 131. 671, scounet W 470,5, schowet [schouuet] W 393, 30, scouwith S 448, 2, schouvigha Sch 545 (8. unten zu bâna), scouninge S, Sch 545 .

In den daneben stehenden formen claw(a) 'backe' W, J

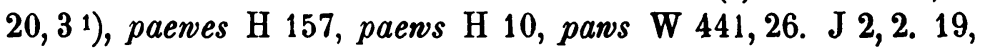
3 , frow $(e) \mathrm{W} 395,15$. 426, 2. 6. 10. 428, 34. 433, 30. 474, 11. H 90. J passim, krawel 'hacke' Sch 535. J 20, 2. 6, daw J 59, 18, thawem moribus H 24 (s. zu thawa fussn.), howen 'gehauen' W 408, 15. 469, 18, hawen, -yn H 111. S 445, 30. 458, 6, scowia, -et, -at etc. W 390, 23. 115,12 . 38. 419, 32. 465, 24. 475, 31. H 42. 43. 46, scawiane H 126, haben naturlich $a(e) w$, ow als schreibungen fur aun, oun zu gelten. So auch in tow 'geräte'2) (doppelform zu dem oben verzeichneten taure), awane, thawa, tawa, -em, taewiane (s. unten zu thawa).

In betreff der bildungen mit altem au (= indog. au oder $o u$, oder für $a$ vor $w^{1}$ oder $w^{2} j$ ) könnte man die frage aufwerfen, ob in denselben der diphthong nicht schon älteren datums als in pauves etc. wäre, indem sich das $u$-element durch einwirkung von $w$ erhalten hätte. Die antwort hierauf musste indessen eine entschieden verneinende sein: au wurde hier, wie im ags., vor $w$ nicht anders behandelt als vor andern consonanten. Wie der alte diphthong im vorags. durchaus zu éá, ie wurde (vgl. iewan, éawan, déaw, Jéan, scéawian, héawan), so erlitt derselbe im vorwfr. durchaus contraction zu $a$; wäre au vor $w$ nicht monophthongiert, dann musste es entweder in allen formen als au erhalten geblieben oder eventuell in folge einer vor der entwickelung des jungeren au aus $a+w$ stattfindenden qualitätsänderung des ersten elementes zu ou geworden sein; nun finden sich aber, wie aus den obigen belegen hervorgeht, einerseits bildungen mit au, andrerseits mit ou, eine

1) Neben dieser aus dem acc. sg. stammenden form steht die ursprüngliche nominativform kle (aus *kla für *klâu für *klâwu) in klêwendene 'das wenden der klauen' (s. über das wort Tijdschr. v. ned. taal- en letterk. $11,308 \mathrm{ff}$.).

2) An der parallelstelle zu H 64 , näml. W 439,17, een schip sonder rêma ende roer ende sonder to $w$. 
erscheinung, die sich nur daraus begreift, dass $\hat{a}$ vor $w+\vec{o}(-)$ und $w+u$ zu $\hat{o}$ wurde, sonst aber erbalten blieb, und aus diesem $\hat{o}$ bez. $\hat{a}$ in der folge ou bez. au hervorgieng (s. auch unten zu trouwaden und sauwen). Vgl. scourvia etc., houweth etc. mit $o u-w$ aus dem in den flexionsformen auf altes $-\breve{o} s(t)$, $-\breve{o} t h,-\breve{o d} d(-)$ bez. $-u$ (1. sg. praes. ind.), -oth (pl. praes.) entwickelten $\hat{o}-w$ für $\hat{a}-w$ (vgl. auch *kouvia zu trou waden); ton: 'geräte' mit ou-w aus dem im plur. mit altem -o (nom. acc.),

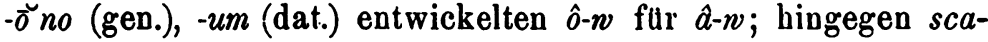
wiane, taewiane, bidauen, ha(u)wen etc. mit au-w aus dem den andern conjugationsformen zukommenden $\hat{a}-w ; \operatorname{tau}(w)(e)$ 'geräte', clau, $\operatorname{claw}(a)$ und tawa, -em 'beweise(n)' mit au- $w$ aus dem den singularformen mit altem $-a$ (gen. acc.) und $-e$ (dat.) zukommenden $\hat{a}-w$ (wegen des letzteren als urspr. $\hat{\imath}-, j \hat{o}$-stamm anzusetzenden nomens, s. zu thawa, beachte man, dass wegen des nach towerie, s. oben s. 359 , und trouwaden etc., s. s. v., ebenfalls vor $w$ (aus $v$ ) entwickelten $\hat{o}$ (und $o$ ) fur $\hat{a}$ (und $a$ ) dieser lautwandel jungeren datums sein muss als die synkope des endungs- $j$, welche dem oben s. 353 erörterten zufolge der genesis von $w$ aus $v$ vorangieng); blaun ( $\hat{o}-w$ bez. ou- $w$ kam nur dem starken nom. acc. pl. fem. auf altes $-o$, dem starken dat. pl. und einigen schwachen formen zu); dau, $t(h) a u$, deren au aus dern gen. dat. stammt (in letzterem auch im pl. verwanten nomen kam den pluralformen auf altes -or nom. acc., -o gen., -um dat., urspr. $\hat{o}-w$ bez. ou- $n \mathrm{zu}$ ); die schwachen verba 1. klasse awane, thawa 'zeigen' mit au- $w$ aus den flexionsformen, die kein $u$ oder $o$ in der endung hatten; nauwer neuter, iauwelik quisque (wegen iouwelik s. zu sauwen) und craul-, krawel mit altem -we-, -wi-. Fur die paunes etc. zu grunde liegende form ist demnach ein anderer endungsvocal als der im ags. pávos begegnende anzusetzen.

Für altes $\hat{e} w, \hat{e}-w$ und $e-w$ sind nach $i \hat{a}$ bez. iou vor $w$ aus $v$ (s. oben zu biliouwa causativ) desgleichen $i \hat{\imath}$, iou zu erwarten. Daher die berechtigung mit ags. fléwsa fluxus (aus *flêwiso), awfr. fliu(e)s- (aus *flên(i)so) zu identificieren, dem als compositionselement des öfters in den busstaxen begegnenden fliu(e)swerp $\mathrm{W}, \mathrm{S}$ u. a. nach folgender belegstelle die bedeutung 'untiefes wasser' (worin einer stehen kann) beizumessen ist (gegensatz onwad vetter, worin einer nicht stehen kann und die 
gefahr zu ertrinken läuft): Fliuesverp, hveerso een man virt veet ende vâsich (nass und schlammig) ..., dī̌ bôte is VIII graet iefta sexasum onswara. Jefter een man vôrd vorpen in een onvad veller, dat (hi) mit âghenen mei siaen hôr himel nêr eerda nêr dine grond rêka mitta hânden nêr mit fôlen, soe schilma him bêla mit eerre lioedwirden ${ }^{1}$ ) trybeet ende oenbrins ${ }^{1}$ ) iefla tolvasum ontswara $\mathrm{W} 463,25 \mathrm{ff}$.

Andere hierher gebörige formen sind: syâ, sion (s. s. v.); *sîwa 'schiessen' (s. zu siâth); syoen 'gesehen' (aus *sewan; für die belege und wegen $i \hat{o}$ für $i \hat{u}$ vor $n$ s. oben s. 358 anm. 4); nwfr. kryoelje scatere GJ = awfr. *kriôlia (für *krialia, das durch vor der iou-genesis stattfindende synkope des mittelvocals aus *kriùvelia $=$ ahd. crewelôn scatere entstand); nwfi. ljuerck 'lerche' GJ $=$ awfr. *liurk(e) (mit î vor $r$ durch anlehnung an die angelsächsischem léwerce entsprechende form *liâwerke, deren $i \hat{u}$ hinwider zui zeit der iou-genesis durch anlehnung an synkopiertes *lîrke erhalten blieb); ioegha, -e, iôghe (flect.) 'ewig' Sch 511. 520.610. 671 (wegen ioe, iô s. unten zu focht), io(u)wigh Sch 663. J 15,5. 18, jouvelike Sch 536; nwfr. sluwg 'träge' GJ (d. b. slîg) = awfr. *slìg (oder mit noch erhaltenem ersten componenten, s. oben 8. 351 anm., *sliug aus *slêvig, vgl. ahd. slêo). Neben ioegha etc. finden sich (e)euwich Sch 671. 674, enig W, Sch 538. 608. 671. 715. 724. Ag 99. 102. J 9, 5. 21, 23. 22, 28, ewelick W, Sch 394. 612. J 50, 12 und ausserdem ewe 'gesetz' (schwaches fem.) W, S, H 20. 21. 112. 148 etc. J 1, 2. 2, 3. 4. 29,1. 45, 7. 46, 71. 60,14. 84, 11 (mit en $=$ $e u-w$ oder $\hat{e}-w$ ? $)^{2}$ ), was zur annahme nötigt, dass einem zwischen $\hat{e}$ und hellem endungsvocal stehenden $n$ die făhigkeit der erzeugung eines $u$ abgieng und demnach primo das i $i$ von *sliug und *iag (woraus ioegha etc. und *înig prototypus zu io $(u)$ wigh) in den synkopierten formen der adjectiva entstanden sein muss, secundo das eu von (e)eurich und ewig, ewelick, ene (insofern bier nicht $\hat{e}-w$ vorliegt) ein ebenfalls aus diesen synkopierten formen und beim nomen aus den flectierten casus

1) Wegen lioedwirden und oenbrins s. v. R.'s Wb.

2) Die existenz letzterer lautverbindung wird erwiesen durch $n$ wfr. ieuvig und ieu 'jabrhundert', welche ihr ieu nur einem auf *êrvig, "êrve zuriickgehenden *ienig, *iewe verdanken künnen (vgl. oben s. 361 über brieune, grieuna). 
(mit altem -un oder analogisch entwickeltem - $a[n]$ ) stammender diphthong ist, der durch anlehnung an das nicht afficierte, aus der nicht synkopierten adjectivform und dem nom. * êwe berrührende $\hat{e}$ vor lubergang in $i \hat{u}$ geschutzt wurde (vgl. was oben s. 360 uber ewnd etc. bemerkt wurde). ${ }^{1}$ ) Ein gleiches eu hatte auch das aus nwfr. sleau 'träge' GJ zu erschliessende awfr. *sleu (wegen euu, d. b. $e^{a} o^{u}$, vgl. oben a. a. 0.; die erhaltung des $e$ als ersten componenten wurde veranlasst durch unflectiertes *slê).

Fôrdêl, fo(i)rndel. In verschiedenen rechtsbestimmungen wird der fôrdêl erwähnt als der platz, worauf das baus eines gerichtlich belangten liegt und von gerichts wegen die eine oder die andere diesen angehende anforderung gestellt oder ihn betreffende gerichtliche handlung vorgenommen wird (s. W 392, 13. 14. 396, 21. 399, 1. 411, 20. 414, 2. 19. 422,12. 15. 394, 33). Den componierenden elementen (fôr-mit vor $r$ gedehntem vocal) zufolge muss mit dem nomen eigentlich und ursprunglich der 'yordere teil' des hausplatzes gemeint sein, $d$. h. der vor dem hause liegende, für die gedachten zwecke geeignete teil. Durch ubertragung aber wurde das subst. auch zur benennung des ganzen hausplatzes und des darauf liegenden hauses, also in der bedeutung 'haus und hof' verwant: Jef hy (der uberfuhrte mörder) naet bêtha nelle, soe aeghmen toe brând ende toe breck toe dwaen, ferdloes to lidsen, hem ende al sŷn gued $w t$ dae fôrdêl (aus seinem haus und hof) toe lidsen ende toe wŷsen, al ônt hy bêth mith fyoerteen pondem etc. W 413,31; Nympt $d y$ tolner onriachte tollen ende him trê schepnen bitingia willet, so aegh di schelta foer alle dâ, deer hi tollen wr laet (auferlegte), fan da fôrdêle (rom haus und hof, d. h. je nach

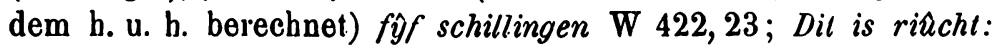
hweerso ma een wîf an nêde nimpı end ma dat claget da frâna, so schelma her folgia mitta aesga ti da fôrdêl (zum haus und hof), deer hiŏ binna is W 391,32; Jef des tyêvis kempa howen wirth, dat dij aesga dat gued dêla schil twischet to jowene; so schel [schil] hij mitter haudlêsene bêta ende foer al dâa, dêr bynna da fôrdêla sint (die zum b. und h. gehören), wederjeld to jowane, alzo fŷr so se jêrich sê $\mathrm{J} 60,22$.

1) Als compromissbildungen aus iouwigh, iouwelik und enig, ewelick begegnen jewegh, jewelick Sch 517.668. J 86, 2 (mit jêw-oder jeu-w?). 
In S 491, 33 steht foirndêl (l. foerndêl) mit foern- = ahd. forn: Foerd soe kêden (lies kêde) wy weduwen ende rềsen ende alla mannalîkum een festen ferd ende in zŷn foirndêl to sitten also langh, unt hy mit riûchte wrwonnen sê.

Als 'privilegium' begegnet dieses fôrndêl in $\mathrm{J} 20,10$ und 22, 1.

Foerfeerd in ende aeck heerdense (die kinder Israel bei der gesetzgebung am berge Sinai) alsoe hêra hoerna hluud;... dae nôrdense soo seer foerfeerd fan da grŷslika bêre (lärm), datter nimmen libba dôrste, eer Moyses van da birgh caem aef W 438, 34 (s. auch die parallelstelle $\mathrm{H} 28$, wo ferfeerd steht). Statt v. R.'s unbegründeter tabersetzung 'bewältigt' ist hier die bedeutung 'in furcht versetzt' geltend zu machen. Man beachte mnl. vervaren, mhd. ververen 'in furcht versetzen' = awfr. fourfêra neben ahd. fârên, as. fâron insidiari, wie mhd. vâre, mnl. vare 'furcht' neben ahd. fâra insidiae.

Foerdwîsa, -fînda, -dêla, -ghaen. Die verba fìnda und wis $a$ stehen in den awfr. (und aofi.) quellen als synonyma $=$ 'ein gerichtliches urteil fällen, gerichtlich erkennen'. Mit rúcksicht hierauf lässt sich für ein mit foerd verbundenes finda oder wisa nach foerdgaen = 'unbehindert', d. b. 'gulltig bleiben'1) die möglichkeit einer bedeutung 'durch gerichtliches urteil fur gulttig erklären' erschliessen, deren gegensatz dann ein mit wrbeck 'zurlick', oppositum zu foerd, componiertes fînda oder wis $a=$ 'durch gerichtliches urteil fur ungultig erklären' bilden könnte. Die eine sowie die andre bedeutung nun finden sich in der tat, wenn auch in uneigentlicher verwendung, $d . h$. bei verbindung des verbums mit der person als passivem subject anstatt mit der sache, bei foerd- und wrbeckwyssa in S 503, $28 \mathrm{ff}$. (in der instruction fur die von den praelaten von Waghenbrugghe angestellten richter [greetman]): hwanneer dae greetman ieft riüchteren bisel wir det in dae prêsteren (man von den 'greetman' auf die priester appelliert) ${ }^{2}$ ), so scelma da papena ponten

1) Vgl. W 433, $32 \mathrm{ff}$. Dit sint da fiouer riùcht (rechtsangelegenheiten) ...., deer (welche) da wird (das zeugnis) schillet nederslaen (nicht gelten lassen); elkers (sonst) om alle ting, deer redelic sint, moet diб wird foerdgaen.

2) Diese aus dem zusammenhang hervorgehende bedeutung des ausdruckes biset nirdet begreift sich bei beachtung des aus de $\mathrm{m}$ citierten 
(rechtsbestimmungen) al tîda foerdwîsa (folge leisten, s. uber diese bedeutung gleich unten), ende deer (wer) dêr toiênst dêth ieft dwaen wol, den scelma wrbeckwŷsa (dessen urteilsspruch soll man fur ungultig erklären); ende wirdith dae greetman wrbeckwŷsd, dae greetman da prêstera cost to staen (für die kosten der pr. aufkommen) ieft hidre terinck ende dat fan hiara pânden 1) te nimen ende iên dat toe hoeden, ende dî, deer hiaerem bilecket (ihr urteil beanstandet), zŷnen (lies zŷne) panda frŷ weer to habben; ende wirden (l. -e) dae greelmaen foerdw $\hat{y} s t$ (wird das urteil der 'greetmaen' fur gulltig erklärt), dŷy, deersie bilecketh hâth, dae prêsteren teringe toe staen ende greetmanspanden dan onbelest to wessen.

Bei verwendung der in rede stehenden composita in weiterem sinne konnte die engere bedeutung 'durch gerichtliches erkenntnis fü gultig, ungultig erklären' ubergehen in 'fü gültig, ungtltig halten', '(nicht) folge leisten'. Fur solches foerdwisa s. die oben citierte stelle; für solches wrbecfŷnda J 24, 2 Dat ma swerren êden, deer naet sint jeffta gaet tojênst da seelsâlicheit, dâ sintma al schyldich to halda [hâlden] foer al tingh ende mey ma maet wrbecfŷnda.

In etwas abweichender bedeutung begegnen foerd und wrbeck in $\mathrm{W} 425,13 \mathrm{ff}$. (und der parallelstelle $\mathrm{H} \mathrm{158):} \mathrm{dal} \mathrm{ma}$ dyne hâldere (den beklagten) aeg foerd to fŷnden an da lioedwarve ... om redelika thing, bihala om tyower thing. Dit is dat aerst fan da foweren: hweerso een man leyt oen da lesta eynde, haetso hi dan dêth bi sînes papa rêde, so aechma da oen-

paragraphen sich ergebenden umstandes, dass derjenige, der von den 'greetman' auf die priester appellierte, eine caution zu hinterlegen hatte. Setta hiess u. a. 'caution stellen' (vgl. W 418, 17 ff. Jefter een schip an dine ouir comt endma hit penda wil, soe aegh hy to gaen mitta schelta ...ende to iariane, datter him dine rôder iona wil ende onderpând setta); daher bisetta eig. = 'wegen (bi-) des richters oder besser des richtspruchs, den man beanstandet, caution erlegen', durch übertragung 'gegen das erkenntnis einsprache erheben'. - Siehe noch in der nämlichen instruction W 501, $23 \mathrm{ff}$. (§ 12) dat da grecthman naeth riuchta scelleth wr desse ienwerdige ponten ...; ende hwanneer mase beseth in dae prêsteren, dis riuchtis naeth to wernen.

1) Wegen der von den 'greetman' bei antritt des richteramtes gestellten caution s. in der nämlichen instruction, S 501,14 ff. (§ 11), dat aider greetman scel selta dae prêsleren honderl ponde pânde. 
spreeck foerd toe fîndane. Dit is dat ôder: hveerso een man sŷn frîahals (freiheit) schil biradia (beweisen) ..., so schilma him ... sŷn frîahals tôdêla, al haet hi dae oenspreeck (auch wenn er kläger ist). Dit is dat tred: hveerso di fria Frese ofte diō fr $\hat{\imath}$ Fresenna willet sprecka on da bannena sinde om een aeft, daltit britzen (ungültig, nicht rechtskräftig, eigentl. kraftlos, gebrochen) sê, ende hi $\breve{a}$ willet bireya (beweisen) ..., soe aechma hiârem foerd to fînden; soe schilma dat aeft gaer dêla. Dit is dat fiaerde: hveerso faeder ende een moeder tyaet (zeugen) tri $\vec{a} k \hat{y} n d e n$, tween sonen ende een dochter, ende hâra ielderen dan stervet, ende hiō dan een man nimpt buta rêda bêdera brôren, soe quact (sagen) da brôren, hiō habbe her gued wrschelt (eingeblisst, s. unten s. v.), omdat hiō et deen haet bita hiâra reed; nw quêth her mond, hiত̆ nabbet naet wrschelt om dine frŷa wilker, deer her ende alla frowen di koningh Kaerl ghâf..., truch dat aeghma da frowen foerd to fînden ende due brôren wrbek, iefla een soen [soene], deer wise lyoed lowiad ende rêdet; ief dae $n \hat{y} s a$ lioed ne connet naet wreen wirda, soe schil doch der frowa riùcht (forderung) foerdghaen.

Hier stehen foerd-, wrbekfŷnda offenbar = 'durch gerichtliches erkenntnis zuerst', bez. 'zuletzt (zur führung des beweises) zulassen', eig. 'd. ger. erk. den vortritt gewähren', bez. 'zurlicktreten lassen'. (Vgl. auch foerdghaen am schluss des paragraphen $=$ 'zuerst berlicksichtigt werden').

Auch beachte man foerddêla = foerdfŷnda und tojênst fŷnda = wrbekfŷnda in J 13,16 Dat dij silter (beklagte) mit lŷcka riucht nyaer is and hyne dij riuchter altŷda aegh foerd to dêlane, byhâla om fyower da eenstigha secken (praerogative), deer allîda myt lŷcka riacht een stal aeghen to habben; aldeerom aegh dij riukchter hy $\vec{a}$ foerd to dêlen, want hy $\vec{a}$ grâten eenst (vorzug) ney da riûcht habbet; jelkirs (sonst) hwŷr so da riûcht ende da prowinge lŷck sint, so aegh dij riûchter tojênst dyn clager to fŷnden. Dit sint da fyower eenstlicka secken: ... dis menscha lesta willa ... frŷheed dis halsis ... dat hellige aeft ... der frowa birnse (heiratsgut); und vergleiche wegen andrer belegstellen fur foerdfŷnda und lobeckfŷnda in der zuletzt orwähnten bedeutung Dat ma dyn sitter schel myt lŷcka riucht. altîda foerdfŷnda, byhala om fiower byschêdelike tingh: ... om een aeft ... om een testament ... om bernlse (heiratsgut) ... 
om een frŷheed dis mannis, dis wives ${ }^{1}$ ) $\mathrm{J} 13,7$; Hweerso da prowinga ende da sykeringa (abwehrung ciner anklage) gaer commet myt lŷcka riâcht, so aeghma da sikeringa foerd to fînden $\mathrm{J}$ 13,19; Hweerso (eine frau oder deren kinder beim leben desjenigen, der ibr die mitgift gegeben hat) dis ... guedes habbet binetten ende bysetten... sonder weerspreek sŷns in êniga rî̀cht ... ende dij sitter (angeklagte) dat claerlich [claerlick] bynîsa mey ..., soe is ma scieldich den sitter mey aldulker festicheed ende bisittinga foerd to fŷnden, ende dat gued to byhalden ende to brûcken to sŷn frŷa willa, ende diin oenspreker [-ir] tobeck J 82, 1.

Focht, fûcht. Die beiden für die 3. sg. praes. ind. zu faen capere verwanten formen (s. fur letztere $H$ 118. W 103, 20. 406, 30. 410, 24. 433, 5. 470, 31. S 497, 31. J 22, 18. 35, 1. $38,1.40,1.44,6.45,17.87,5$, fur die andere H 49. 106. 176. 240) stehen für älteres, regelrecht entwickeltes * fêth (= aofr. fêth aus*fen hith) als neubildungen nach tiòcht W, S, H 164. 165. 176. J 48, 2. Sch 335 und tiucht W, H 47. 154. J 3, 3. 47, 5. 7. 13. 50,41. 42. 72, 7, siòcht $\mathrm{H} 88$ und siucht $\mathrm{W}, \mathrm{J} 2,14.13,6$. 58, 25. 71, 6. 72, 8, der 3. sg. praes. ind. zu tiaen 'ziehen', siaen 'sehen'. Bei der begrtundung des hier angesetzten iò (mit kurzem $o$ ) und $o$ erlaube ich mir zu gleicher zeit einiges uber die schicksale von i $\hat{u}$ im wfr. zu erörtern.

Besagter diphthong (fur íu, das germanischem eu entspricht oder westgerm. eu bez. iu aus $e$ bez. $i$ vor $w^{1}$, oder durch einwirkung eines folgenden $w^{2}$ oder $w$ aus $v$ entstand [8. oben 8 . $353 \mathrm{ff}$. und 378], oder sich, wie im aofr., s. Gramm. § $39 \mathrm{mit}$ nachtrag und $\$ 36$ und 37, durch brechung vor cht, chs, ks, bez. durch $u$-oder $w$-epenthese entwickelte) ist im awfr. zum teil erhalten geblieben, zum teil in $i \hat{o}^{2}$ ) oder io ubergegangen und zwar nach folgenden gesetzen:

1) In bezug anf die hier in den zwei vorangehenden und dem erstfolgenden paragraphen zu tage tretende rechtsregel vgl. J 13, $21 \quad D i$ sitter is nyaer (näher) mit lycka riùcht to ontgaen (sich eidlich von einer anschuldigung zu reinigen) dan dy oenspreker; dy ongong is nyaer dan dy oenbrengh (die eidliche anklage).

2) D. h. iôu, wie sich schliessen lässt aus dem im jüngeren nwfr. stattfindenden übergang zu iû oder î̀े (s. unten). Die reime lioed: hluud 
$\alpha)$ î bleibt vor einfacher tautosyllabischer muta und in den sub $\beta, \gamma, \delta$ und $\zeta$ gemeinten ausnahmen;

$\beta$ ) es wird zu iou vor $w$ (wegen der belege und der durch anlehnung entstandenen ausnahmen s. oben s. $351.353 \mathrm{ff}$. und $378 \mathrm{f}$.);

$\gamma$ ) es wird zu $i \hat{o}$ (in unbetonter silbe $i o$ ) vor heterosyllabischer muta und im wortauslaut, im letzteren fall aber nur, insofern nicht das $i \hat{\imath}-w$ einer verwanten oder einer nebenform schutzend einwirkt (schreibung ioe oder io);

$\delta)$ es wird ebenfalls zu $i \hat{o}$ vor tauto- und heterosyllabischen $l, r$ und dent. $n$, insofern nicht das iu-n einer nebenform schutzend einwirkt (schreibung ioe und io in offener, ioe in geschlossener silbe);

ع) es wird zu iò vor $n g, n k$ (constante schreibung io);

5) es wird ebenfalls zu iò vor tautosyllabischem cht, insofern sich nicht analogisierender einfluss geltend machte, und vor $k s$ sowie $k k$ (constante schreibung $i o$ ).

Fur die verba und nomina, in deren flexionsformen tautosyllabische einfache muta mit heterosyllabischer, bez. $c h-l, k-s$ mit cht, $k s$ wechselten, ist nach $\alpha$ und $\gamma$ bez. $\alpha$ und $\zeta$ die entwicklung von doppelformen nebst ausgleichung etwas selbstverständliches.

Belege für $\alpha$ : tiûflig 'gestohlen' W 397, 26, tiûftigia 'stehlen' J 60, 9.16 mit p. p. tiuftigad, -eged W, J 59, 4, tiuchte 'diebstabl' H 74. 135. 141. 165. 169, tînchlig H 56, tîuchtgath 'gestohlen' H 93 (mit $c h$ aus $f$ vor $t$ ); tiuester, tynster, -lick, -nisse W 37, 11. 47, 1. J 7, 9. 13, 46. 21, 26. 27, 14. 46, 42; fliu(e)swerp W, S, H 226 (s. oben s. v.); flîs in flyûscâper, fliuesdên (oben s. v.); bynsterlick 'verwirrt' J 12, 8; $\operatorname{syu}(e)(c) k t(e) \mathrm{J} 3,13.19,2$. 45, 11. 46, 16. 53. 62. 73, 2 mit bisiuckt 'krank' J 73,1. 2.

Belege fur $\gamma$ : nió(e)gen 'neun' mit nio(e)genda, -têna etc. W, S, H 74. 88. 93. 95.138 etc. Sch 603. 709. J 12, 8. 56, 1 etc.; hyôda, hioede 'heute' W, S, H 24. 299. J 50, 24 mit hiôde-, hioedlik H 116. W; *griôde 'narbe' (s. oben s. 356).

Belege fur $\alpha$ und $\gamma$ (das durch ausgleichung entstandene $i \hat{o}$ wird in geschlossener silbe dargestellt durch ioe, selten durch

W 438, 25. 26, lioede: hlûde H 28 sind nicht beweisend, weil hier urspr. liued(e) (s. unten 8. 385) gestanden haben kann; vgl. riochte: tachte $\mathrm{H} 168$, wofïr an der parallelstelle riùchte: tûchte [tuchthe] steht. 
io): liu(e)d, lywd nom. acc. pl., lyuedem, -en (mit alter, in der historischen periode nahezu normaler apokope der endung) nebst liu(e)dgârda, -ferd, -ware, -wirden(e), -werden(e) W 49, 27. 79, 15 (v. R. falsch lioed-). 409, 11. 416, 10. 422, 17. 423, 32. 424, 8. 426, 23. 428, 28. 471, 1. S, H 165. 254. J passim und lioed, -e, -a, -ena, -um etc. nebst lioedgârda, -frede, -ware, -merck, -wirden(e), -werden(e) W, S, H und J passim; biat(h), bynth '(ge)bietet' W 404, 18. 412, 9. 413, 26. 414,6. J 1, 28. 2, 8. 19, 6. 20, 12. 23,11. 56, 1 und bioel(h), biôt(h) W 412,17. 418, 22. 435, 34 . J 48, 1. 2. H 102. 121. 136. 137, foerlŷust $J 2,20.56,1$ und foerlyoest, -lyôst J 3,16. 25, 27 (das iô stammt aus *biôdes(t), -eth etc.; nach den citierten formen und den danach anzusetzenden

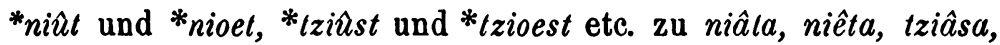
tziêsa etc. bildete sich neben siuth 'schiesst', dem nur î̀ zukam, s. unten s. v. siôlh, sioet); bilywda, bitiueth 'bedeuten' J 8, 1. 15, 35. 81, 2 und biliôda, -en, biliôlh, bithioet W, H 20.170. $\mathrm{J} 27,1$; nyoed und nyoedsecken ${ }^{1}$ ) (as. niud; ein neben nyoed $\mathrm{zu}$ erwartendes niued kann ich nicht belegen); crywse cruci $\mathrm{J} 56$, 12 , cryâsdey, criûcsdei $\mathrm{S}, \mathrm{Sch} 534$, crynsgade 'ein kreuz tragende' $\mathrm{J}$ 20, 8 (eine daneben zu erwartende form mit $i \hat{o}$ habe ich nicht notiert); tiuech, tiûges, -a 'zeugnis', tîuga 'zeuge', tiûga, -en, -e, -et 'zeugen' J passim. W und tioech, tiôga, tio(e)gha, -ane etc. $\mathrm{W}, \mathrm{S}, \mathrm{H}$ passim (danach auch tywcht $\mathrm{J} 8,9$. 18, 23 und tioeght, tioecht W 476, 1. H 123. 165 mit iû, iô für lautgesetzliches iò, vgl. oben 5); ioegha, -e, iôghe (s. oben s. 379; eine form mit iu kann ich nicht belegen); io, ioe dat. acc. des pron. pers. W, H passim. J 15, 27. 55, 4. 58, 37 (daneben auch $i \hat{\imath}$, in $\mathbf{W}$ 430, 14. 431, 21. 432, 12 nach dem poss. $i \hat{u} n(e)$, das sein î für iou hinwider der anlebnung an das pers. verdankte, s. oben 8. 351 anm.; nach dem nwfr. aus der enklitischen form stammenden $j o ̀, j \grave{u}$ ist auch für das awfr. die wenigstens teil-

1) In in need ende yn nioed cen help habba Sch 708; ende bystandt toe dwaen in need ende in nioed und ende hem hilplyck syê in need ende in nyoed Sch 709; hwa dit naet en dêt (wer zur leichenfeier des verstorbenen schutzenbruders nicht beisteuert), en hael pont vorberd, need ende nyoed wiseid Sch 603; Hweerso een riüchter naet mey komma fan freesheed sŷn lŷf off feen oers needsecken off nyoed secken,... so mey er sŷn jurisdictionen ... byfelle een trowe man J 2, 24. Die formel hiess eig. 'necessitas et studium', doch ist in derselben das zweite wort offenbar zum synonymon des ersten geworden. 
weise verwendung von $i o$, iù zu vermuten). ${ }^{1}$ ) Wegen $*_{r i}$ s. oben s. 355.

Die bei vielen formen $\mathrm{zu}$ tage tretende doppelherschaft ist auch im älteren nwfr. (s. GJ) zu beobachten: ljue und ljoe 'leute', betjütte 'bedeuten' mit kürzung des zweiten componenten vor dem $t t$ der praeteritalen formen und betjoede. Doch tjungje (d. b. tjûgje) 'zeuge', tjung 'zeugnis', tjungje 'zeugnis ablegen', und, wie im awfr., krjues, krjuesgje 'quälen'. Ausserdem wjueck ala GJ, slung, stjung, stjungje (s. oben s. 379. 359) und das etymologisch dunkle tjoe $(d)$ 'sonderbar, unwol, erzürnt' GJ. Gegentiber nyoed stehen bei G. Japicx njoe und njue 'bereitwilligkeit, geneigtheit'. Ob diese aber auf alte njoed und *niued zurückgehen, dürfte fraglich sein, weil es auch denkbar ist, dass neben altem, durch verdrängung der $i \hat{u}$-form zur alleinherschaft gelangten nyoed eine neubildung entstanden wäre auf demselben wege, auf dem sich awfr. tioester, tiôster H 84.120, flioes-, fliôswerp W 451, no. 10.11. H 122, sioecte, syôkte H 83. $\mathrm{J} 73,1$ (die hs. hat hier syoket) neben urspringlichen tiuester etc. (vgl. auch nwfr. tjoester neben tjuester GJ) und nwfr. (GJ) njueggen neben njoeggen, bij djue 'täglich' neben bij djoe (mischform aus dey 'tag' und hyoe) entwickelten, d. h. durch analogiebildung nach den von rechtswegen mit beiden diphthongen gesprochenen formen. Man beachte noch nwfr. (GJ) njoet und njuet 'zahm, rubig' (an. nýlr utilis, mittelostndfrk. niute utilis Teuthonista). Die jetzige sprache kennt ausser im auslaut nur noch $i \hat{u}$ bez. îla oder in einigen formen mit vor zweifacher oder tautosyllabischer consonanz gekurztem iù; also lju, betsjütte, njô 'freude', tsjüster, njùggen, njût 'zahm', $t$ sjù (d) 'schlecht,

1) Der lange component erhielt sich im nwfr. noch in dem nach dem muster von $m \hat{\imath} n$, dîn (thîn) neben $m \hat{\imath}$, dî, (thî) aus iô gebildeten possess. jôn GJ $=$ awfr. ioen in ioene acc. sg. masc. W 431, 14. H 171 (2 m.). 173, ioen(e) nom. acc. pl. H 119. W 430, 9, iônre dat. sg. f. W 432, 5 ( $\nabla$. R. las an den drei stellen in $W$ falsch ioeue, ioeu, ioure), joen dat. oder acc. sg. ntr. J 6, 4. (Vgl. auch noch ioere dat. sg. f. H 113 (2 m.) nach analogie von ioe neben îे statt $i \hat{u}(w) r e)$.

Für die nom. sg. f. hī̄ und $d i \bar{o}^{-}$sowie für den instrument. diô in bidiô W 406, 29 hat nach aofr. hiō thiō (Gramm. § 242. 246) genesis aus *hio, thio mit geschwächter endung bei dissyllabischer aussprache zu gelten. Die heutige sprache verwendet neben $(h) j o$ auch ein nach dem person. 2. pers. $j \grave{u}$ neben $j \grave{o}$ gebildetes $(h) j \dot{u}$. 
böse' '), sowie (h)jî̀ 'heute', grûade 'narbe', ebenso gut wie flûss 'vlies', bjüster, woüuk 'flugel' etc. und tsjûge 'beim tausch den vollen wert für etwas erhalten' (mhd. ziugen 'die kosten wovon

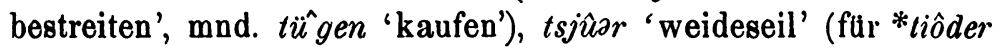
aus *tiudir; vgl. nordfr. tjudder Outzen 359 , und in GJ tjürrie 'mit einem weideseil festbinden'); ferner rjù und $j \grave{u},(h) i \grave{u}$, doch jò, (h)jò (s. oben).

Awfr. belege für $\delta$ sind oben s. 356 anm. 1 und 358 anm. 4 verzeichnet. Die ausnahme fyuer, fywr J 58,40. 59, 17 ist auf eine linie $\mathrm{zu}$ stellen mit tioester etc. veben tîuster etc. Das ältere nwfr. (GJ) hat dem awfr. entsprechend snioen, fjoer, djoer, djoerte, stjoere, sjoene 'gesicht', sjoen 'gesehen', sowie kryoelje (oben s. 379), stjoer 'unfreundlich' GJ (awfr. *stiôr(e) aus *stiuri), und das etymologisch dunkle njoer 'unfreundlich' (woneben auch unurspr. njuer); doch frjuen als analogiebildung für altes friônd. Wegen der ausnabmen scriuen, sniûn, ljuerck, sijuwn und juwn s. oben s. 355. 356. 359. 379 und unten zu geva. In der heutigen sprache gilt auch hier bald immer $i \hat{u}$ bez.

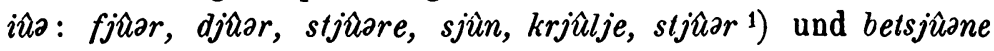
'behexen' (ags. týnan 'schaden zufugen'), sowie ljûark, stjûn; doch snjòn mit kürzung des zweiten componenten, wie in frjòn, das auf ein im älteren nwfr. neben frjuen stehendes frioen $=$ awfr. friônd hinweist.

Belege fur $\varepsilon$ : sionga, -ane, -en, -t W, H 67. 103. J 77,9. Sch 709; dyòncker J 12, 8. 22, 20. 24, 21 (einmal dywnker J 8, 1 als neubildung nach dem muster der doppelformen mit $i \hat{u}$ und io, s. unten $)^{2}$ ); nwfr. (GJ) sjònge, stjonckje, und nach jetziger aussprache auch sjonge, stjonke.

Belege für $\alpha$ und $\zeta$ : die oben s. 384 verzeichneten tiûcht, siâcht und tiocht, siocht, flîcht 'fliegt' $\mathrm{W}$ und fliocht $\mathrm{H} 29$, sowie fliacht 'flieht' W (eine nebenform fliocht kann ich nicht belegen; $i \hat{u}$ stammt aus der 2. sg. mit enklitischen pron. *tiuch-stu

1) Reminiscenzen an den alten lautwert bewahrt indessen die landlïufige orthographie in tsjoed, hjoe, sjoen, betsjoene etc.

2) Der diphthong entwickelte sich in einer nebenform $=$ aofr. diùnk, an. dekkr (Aofr. gr. § 37) und gieng daraus in die bildung mit suffix -(e)r über. Altes * jinkwra- musste sein interconsonantisches $w$ verlieren (vgl. ahd. fatureo $=$ aind. pitrvyas 'ohein') und eine form mit $i$ ergeben, die in der tat im awfr. dynker J 13,9.13 vorliegt. 
etc.; wegen tywchl, tioecht 'zeugt' s. oben s. 386); rîcht subst. und adj. W, H. 1. 20. 22. 24. 40. 68. 81. 159. 256. 299. J passim und riòcht Sch 334. H passim. J 1,9 (iò aus der unflectierten form, îu aus riûchta, -es etc.); rî̀chta verb. W, S, Sch 335. 394. J 58, 5 und riòchta Sch 394. S, H passim (io aus riòcht 3. $\mathrm{sg}$. praes. ind. und p. p. und *riòchtst 2. sg.); fîchta 'fechten' W, J 2, 28. 13, 13. 26, 25. 58, 6. 66, 5. 70, 1. 71, 3. 75, 3 und fiòchta H 73. 84. 85. 88. 136 etc. Sch 335. 394 (iò aus fiòcht, *st) 1); sliûcht 'schlicht' J 15, 79. 17, 15. 17. 21, 25. 22,6. 37, 7. Sch 732. 741 (sliòcht kann ich nicht belegen); liòcht 'niedrig' (8. unten s. v.; liûcht kann ich nicht belegen); siòchte W 43,6 (mit iò aus der apokopierten form; ch aus $k$ vor $t$ ) ${ }^{2}$ ); im nwfr. des 17. jb. nach GJ tjòcht, fljuegste, fijuecht, sjòcht und sjuecht als 2. 3. sg. (und durch analogiebildung tjòg, fljueg, sjog 1. sg. und imp.); rjuecht, sluecht (in dem Zuidhoekschen in Wassenberghs Taalkundige bijdragen herausgegebenen denkmal rogt, slogt 1,154. 155. 156. 157.164. 166 mit schwund des ersten componenten, 8. oben s. 351 anm.), fjuechte, sjòcht(e), ljuecht lux ( = mhd. liuhte, doch ntr. gen. nach dem muster von liacht), ljuecht(j)e 'leuchten' (im Zuidhoekschen denkmal liogt 1, 154. 160; im Molkwerumschen von Wassenbergh herausgegebenen ljuocht und vorliûglen 1, 144), tjock 'dick' (vgl. aofr. thiuke, Gramm. § 37); in der jetzigen sprache tsjücht, -st, fljücht, -tst, sjùcht, -st (und t.sjüch, fljùch, sjüuch 1. sg. und imper., tsjügge,

1) Ebenso in den durch anlehnung an die praesensformen gebildeten fuchten (fiuchten) W 440,20, fiochten $\mathrm{H} 111$ praeter. und fiuchten $\mathrm{W} 57$. 27 p. p. für fuchten W 408, 20, fochten $\mathrm{H} 66$ und fuchten W, J 55, 4. 58, 6. 8 .

2) Wie in wyêcht 'weicht' W 435,3. 4 (v. R. unrichtig wyêckt) (zu wyêka H 22 = aofr. wiakka, s. Beitr. 14, 277), brecht 'bricht' W 426, 33, sprecht 'spricht' W 435,12 (v. R. falsch spreckt), brincht 'bringt' W 432, 28, sêcht 'besucht' H 37 (vgl. tiber das nämliche cht im aofr. Gramm. § $267 \zeta$ ). In der regel aber erscheint $k t$ : wyêkt, wieect $\mathrm{W} 435,33$ (v. $\mathrm{R}$. falsch wyckt). H 22 (2 mal, Hett. falsch wreect), biswîct H 46, sekt W 390, 34, breckt, spreckt, brinckt etc. überall passim; ebenso die oben verzeichneten siùkte, sioecte, syôkte. Ob hier indessen immer ein (durch anlehnung hergestelltes oder nicht afficiertes) $k t$ vorliegt, ist nicht ganz sicher, denn (un)riùct W 389, 10. 390, 32. 396, 34. 435, 3. 5. 8. 16. 19, fiùct W 435, 20 (wo v. R. falsch (un)riucht, fiucht abdruckte) weisen darauf hin, dass wenigstens in der incunabel mitunter (nach dem muster von spreckt, breckt etc. mit aussprache sprecht etc.) -c(k)t zur darstellung eines -cht verwant wurde. 
fjügge, sjügge pl. praes.) sowie tsjòcht, -st, fljòcht, sjòcht etc. (und tsjoch etc.), rjüucht, sljücht und rjòcht, sljocht, fjüuchtsje (jü mit kürzung vor zweifacher consonanz), sjòcht, ljòcht, tsjòk und $t s j \hat{u} k$ ( $j \hat{u}$ aus der apokopierten form mit vereinfachter geminata), mjükse 'mischen' (wegen $x<$ sk vgl. im engl. to mix $=$ ags. miscian) 1), tsjòksel 'wagendeichsel' (ahd. dîhsala; iò aus *lhiòx-lfur * (hî $\left.x-l-)^{2}\right)$, mjokssje 'die rinne des kuhstalls von mist reinigen' (aus *miuhsōjan denominativ zu *miuhs = ags. meox <* mihs).

Vgl. ubrigens noch vereinzeltes ryoecht Sch 609 mit $i \hat{o}$, wie in tioester etc. (s. oben s. 387), nwfr. ebenfalls seltenes (GJ) sljoecht, sjoecht, fjoecht.

Freed. Dem nwfr. freed 'freitag' entsprechend steht in den awfr. denkmälern in der regel (op) den freed, dis (des) fredis, freeds $\mathrm{J} 3,6,15,27 . \mathrm{H} 37.126$ und an den im Wb. citierten stellen; daneben ausnahmsweise frîedei $\mathrm{H} 126=$ aofr. frî(g)endei (Gramm. § 104). Der gedanke an rein phonetische entwickelung des wortes ist selbstredend ausgeschlossen. Es liegt volksetymologie vor, d. b. anspielung an den frieden, die versöhnung mit gott, welche durch Christi an einem freitag erlittenen tod erfolgte. Dass im gegensatz zum normalen gen. sg. ferda W 107, 4, raef-, strŷdferda (-farda mit $a$ aus $e$ vor

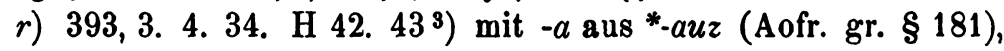
woneben selten ferdis $\mathrm{S} 502,36$, der tagesname nur mit der endung - $(e) s$ begegnet, ist begreiflich mit rlucksicht auf das bei den andern tagesnamen (mit -deis) verwante genitivsuffix. Wegen der neben ublicherem ferd $(e),-a$ etc. pax etc. W, S, H passim. J 2, 28. 58, 8. 59, 2. 15. 78, 3 stehenden älteren form

1) Die endung des fries. verbums -e (nicht $-j e$ ) weist auf die 1 . schwache flexion hin ( $\mathrm{vgl}$. ahd. misgen, p. p. gimiskit). Die brechung entstand also in den synkopierten praeteritalformen, denn das $-i,-j-$ des praesens verhinderte die brechung (8. Aofr. gr. § 39). dîhsila).

2) Woneben auch tiksel (aus einer form mit $-i$ - nach $h s=$ ahd.

3) v. R. übersetzt die composita unrichtig durch 'raub-, streitfrieden'. Es ist hier die rede von einer gebühr, welche der durch gerichtlichen zweikampf eines diebstahls überführte dem 'grewa' zu entrichten hatte, also eines ferde (für die bedentung 'geldstrafe' dieses nomens s. das Worterb.) wegen des diebstahls und des vom 'grewa' erteilten rechts, den process durch zweikampf $\mathrm{zu}$ beenden. 
ohne metathesis frede, $-a$, freed $\mathrm{s}$. die im $\mathrm{Wb}$. verzeichneten belege sowie H 24. 25. 70. 91. 93. 94. 96. 99. 104. 113. 119. 121. 133.135 etc. etc. J 5, 1. 66, 5 .

Gera (die flexion des alten). Diese flexion bietet das denkbar buntscheckigste bild:

inf. und gerund. geua W 425,4. H 177. 178, iewa H 174. 177, gewane, geuuane H 180. W 388,8, iewane H 30. $180-$ io $(u) w a$, -ane, -en $\mathrm{W}, \mathrm{H}$ passim - iaen inf. $\mathrm{W}, \mathrm{S}, \mathrm{H}$ und J passim, îa, iae inf. W, S, J 7, 2. 8, 1. 44, 10. 12. 18. 45, 3. 47, 8. 55, 1. 59,21. 67, 1, iâne, iaen ger. W, H 115. J passim. Sch 535;

p. praes. jaen J 28, 18 ;

1. sg. praes. ind. jowe Sch 517 - 3. sg. ieft $\mathrm{H} 176$, gheeft Sch 603 - iout, iont W, H 72. 92. 149. J passim;

3. pl. praes. ind. iewath H 86 - iowat $\mathrm{H} 86$ var. iaet(h), jaed W 49, 25. J 51, 4. 82, 9. 14 ;

opt. praes. gewe, geue W 388, 24. H 178 - io(u)we, iou W, S, H 64. 166. 173. J 17, 3. 19, 3. 25, 38. 26, 5. 8. 9. 59, 21. $82,14.84,17.19$;

3. sg. praet. ind. iêf H 27. $174-$ gaef W 430,5. 438, 26, gâf (so anzusetzen nach gaef) W, J 8,7 - iouwe H 26. 108. 159 - iô, ioe W, J passim - ioed H 2. 25. 26. 30. 63. 64. 68. 73. 75. 81. 82. 162. 166 etc., ioede $\mathrm{H} 168-j o \hat{g} \mathrm{~J} \mathrm{1,} 46-$ ioef $\mathrm{H}$ 80. 162. 168;

praet. ind. pl. und opt. io(u)wen, -e W 430, 17. H 44. 59. 168 - iô W 434,4;

p. praet. io $(u) n(e) n$, joun W, S, H 2. 117. 134. 154. 168. 170. 174. J passim.

「Man beachte auch die in den aofr. texten P. und E. Sgr. begegnenden, aus den wfr. nachbardialekten entlehnten iova, iôf, iowen; s. Gramm. $\S 7 \beta$, wo die formen jedoch falsch beurteilt sind.]

Wegen der praesensformen mit $o(u) n$, ou s. oben s. 360 (iout 3. sg. ind.. analogiebildung); wegen des wurzellautes in geua, iewa etc. 8. 362 ; das $g$ derselben fur $j$ wurde hergestellt durch anlehnung an das praeter. mit $g$.

Fur iaen, iaeth ist selbstredend nicht an rein phonetische entstehung zu denken. Ebenso wenig aber wäre eine praeteritale form oder der opt. praes. fur die entwickelung der 
neubildungen verantwortlich zu machen. Wir sind demnach für die deutung derselben auf die allein ubrig bleibende möglichkeit einer von der 2. und 3. sg. ind. ausgegangenen analogisierenden einwirkung angewiesen : mit andern worten, es erhebt sich die frage, ob sich die ehemalige existenz von zu geva(n) oder ieva(n) gehörenden flexionsformen für die 2. 3. sg. ind. plausibel machen lässt, welche mit der 2. 3. sg. praes. ind. der formell mit iaen, iaeth ubereinstimmenden verba gaen, staen W, S, H und J passim, pl. ind. gaet $(h)$, gaed W 432, 6. S, H 76, 91. 172. 300. J passim, staed $\mathrm{W} 433,36$. H 25. 141. 176, d. b. mit *gês $(t)$, geel(h), geed S, W 39, 22. 393, 12. 411, 30. 415, 33. 421, 14. 427, 4. H 38. 45. 90. 117. 153. 162. J passim,

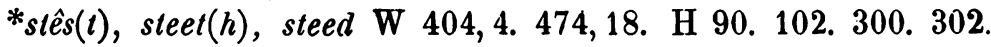
$J$ passim zusammenfielen. Und in der tat scheint mir dies der fall zu sein: aus *gewis $(t)$, -ith oder *jewis( $t)$, -ith mit analogischem $w$ für $v$ (s. oben s. $353 \mathrm{f}$.) mussten in der periode, worin die synkope des $n$ vor $-i$ wirkte, *gês( $t)$, *gêth oder *iês $(t)$, *iêth hervorgehen.

Der inf. $i \hat{a}$, iae ist als compromissbildung aus ieva und iaen zu fassen.

Das praet. ieff steht auf einer linie mit seet, spreek etc. (s. zu quaen).

Gaef erklärt sich folgendermassen: neben altem *bed, *bêdun (s. für letzteres bêden $\mathrm{H}$ 64. W) hatte sich zu bidda durch vermittlung des zu diesem verbum und zu *biadda gehörenden p. p. *bedan, -en (wegen des e der 5. klasse vgl. seten H 54, setten $\mathrm{W}, \mathrm{H}$ und $\mathrm{J}$ passim, wessen $\mathrm{W}, \mathrm{H}$ und $\mathrm{J}$ passim, metten J 25, 35; wegen des $e$ der 2. klasse s. beden W, S, H 160. 163. 166. 169. J passim, bisletten, schetten, wrlern etc., $\mathrm{Wb}$.) und der bedeutungsverwantschaft (biêda, biada = 'gebieten' und 'bieten') die aus der 2. klasse entnommenen bildungen baed 'bat' W 19,7. H 75, bâden, baeden 'baten' W 433, 14. 439, 30. H $65^{1}$ ) (neben bêden W, H 64) entwickelt ${ }^{2}$ ); nach diesem so.

1) Vgl. zu biâda das praet. sg. baed(e) W, H 66. 94. 161, das praet. pl. baeden $\mathrm{W}$ (und den opt. baed J 1, 17; doch bedin $\mathrm{H} 80$ ). Wegen des aus dem sg. in den plur. eingedrungenen vocals vgl. tagen $W$ (neben teghen $\mathrm{H}$ 64), flaegen $\mathrm{H} 27$ (neben flegen W).

2) Das gleiche praet. der beiden verba rief auch ein p. p. baeden 'gebeten' J 17, 11 hervor nach dem zu biàda gehörenden p. p. baeden W, 
baed neben *bed bildete sich von dem verbum, das hinsichtlich seiner bedeutung mit bidda in enger bezietung stand, gaef neben ${ }^{*} g e f .{ }^{1}$ )

Die 3. sg. praet. ind. ioune (wegen des -e gleich unten) ist neubildung nach dem plural. Das praet. sg. iô, ioe und der opt. $i \hat{o}$ verdanken ibre existenz der analogiebildung nach der flexion der 6. klasse: iô neben iae(n) nach stoep etc. neben stapa etc. (mit tonlangem vocal; vgl. auch die s. v. hliâpa besprochenen neubildungen hloep und hliâpa).

Desgleichen sind ioed, ioede (3. sg. ind.) und $j \hat{g} g$ als analogiebildungen zu fassen: ioed neben iaen nach stoed $\mathrm{W}, \mathrm{H}$ passim. $\mathrm{J} 17,16.35,1$ neben staen; jôg neben iaen nach sloegh $\mathrm{W}, \mathrm{J}$ 44,1. 50, 47. 64, 12 neben slaen. (Für ioed vgl. auch siôde 'sah' H 26, siôden, sioeden 'saben' H 54. 65, sioen H 24 mit assimilierung des $d$ mit $-n$ fur -en, sioede 'sähen' H 55 neben siaen; wegen des $-e$ in der 3. sg. ind. iouwe, ioede s. noch ausser dem zuvor citierten siôde die 3. sg. ind. praet. baede $\mathrm{H}$ 66. 94. 161, wârde H 102, biholpe H 1, screwe $\mathrm{H}{ }^{2}$ ), scrioune H 26. 28. 30. $\delta 0.81 .159$, schêne $\mathrm{H} 28$, und beachte die nämliche erscheinung im mhd. und mnl.). ${ }^{3}$ )

loef ist compromissbildung aus iô und iêf.

S, H 81. 163. 164. 166. J 5, 4. 15, 27. 43. 61. 68. 22, 15. 16. 30, 17. 32, 21. 46,1. 64, 17. 80,1, das sich nach dem muster der doppelformen mit $\hat{a}$ und $e$ im praet. (s. oben $8.392 \mathrm{anm}$. 1) neben der participialen form mit $e$ (s. oben im texte) entwickelt hatte.

1) Aus diesem $g$ geht hervor, dass der process schon vor der genesis des $j$ aus $g$ stattfand. Ausser gaef und baed, baeden finden sich noch von anderen verben 5 . klasse praeterita mit $\hat{a}$, nämlich saegh, saeghen 'sah, sahen' W, J. 30, 17 zu siaen nach analogie von taech W, H 135, lágen (8. s. 392 anm. 1) zu tiaen 'ziehen'; lày 'lag' W, das neben lêy H 25 (aus lêg wit $\hat{e}$, wie in seet, spreecle etc., 8. zu quaen) aufkam durch einwirkung von seiten des im plur. nach dem muster von sacghen veben ursprünglichem *sêgen als doppclform zu altem *lêgen entwickelten *lâgen; und waes 'war' W 5, 5. 397, 18. 412, 20. 31. 32. 429, 31. 437, 9. 439, 19. 440, 26, das für das überall passim erscheinende ursprüngliche was (= afr. was, Gramm. $\& 267 \beta$. $272 \alpha$ ) eintrat in folge des umstandes, dass bei dem fehlen sonstiger praeterita sg. mit $a$ und der verhältnismässigen häufigkeit von praeteriten mit $\hat{a}$ (nach der 2 . klasse) die isolierte form sich nach letzterem typus umbildete. Nach waes entstand wâren W 427. 2, H 170 für normales und urspr. wêren.

2) Vgl. oben s. 361 .

3) S. meine Mnl. gr. $\S 161$ und Vondelgr. $\S 55$.

Beiträge zur geschichte der deutschen sprache. XIX. 
Das praet. pl. iouwen gieng auf dem oben s. $353 \mathrm{ff}$. be sprochenen wege aus *gê- oder *iêvun hervor. Der opt. iou(we) stammt aus dem ind. pl. (aus einer der vorstufen zu iouwen).

Das p. p., wofur bei ungestörtcr entwicklung ieven zu erwarten wäre, entstand durch anlehnung an eine der vorstufen des praes. iouwa.

Die heutzutage gangbare flexion des verbums ist ( $\mathrm{Bl} \mathrm{160):}$ inf. jaen; praes. ind. ik jon (d. b. jou) oder jaen (nach gean, stean 1. sg. neben gean, stean inf; vgl. die gleich gebildete 1. sg. dwaen, slaen neben dem inf. dwaen, slaen), du jowst, hy jowl, pl. jowe (mit apokope des $t$ fur *iounet aus iounat) oder jâne (neubildung nach der 1. sg., von welcher sich in der starken und der schwachen flexion 1. kl. in folge der apokope des -e in der 1. sg. und des $-t$ im pl. die pluralform nur durch die endung - $e$ unterscheidet; vgl. auch den gleich gebildeten pl. geane, steane, dwâne, slâne); praet. joech, joegen; p. p. jown.

Bei GJ stehen: inf. jaen (jean); ick joun, hy jount, pl. jâne; praet. joe und joegh, pl. joene; p. p. juwn (d. b. jûn, das, durch synkope aus *juwen entstanden, durch einwirkung letzterer form sein $i \hat{\imath}$ vor $n$ erbielt und nicht in $i \hat{o}$ umwandelte; vgl. juun und sniôn, oben s. 356 f.).

Grêta. In der bekannten, in W $410 \mathrm{ff}$. beschriebenen processordnung begegnen unter andern die formeln $N \hat{u} q u \hat{e} t h d i$ man (d. b. der beklagte), hy ne thoer (brauche) aldus $g r \vec{e} t^{1}$ ) hôr (weder) iechta nêr bisecka 412, 4; Jef hy quêth, dat hy aldus gr $\vec{e} t$ ne thoer hôr iechta nêr bisecka 414, 2; So aegh hi aldus gr $\vec{e} t$ aider (entweder) iechta iefla bisecka 418, 4; (Nu ist rîcht) dat hi aldus gr $\vec{e} t$ anderda (sich verantworten) schil toe seckwird 419,4. v. R.'s fassung dieses gr $\vec{e} t$ als 'klage' (i. v. $\mathrm{gret}$ ) ist $z u$ verwerfen, weil ein subst. hier nicht am platze wäre. Das wort ist p. p. zu grêta 'anklagen'.

Nach v. R. soll ferner diesem verbum nur die bedeutung 'anklagen, auf etwas klagen' zukommen. Es steht aber an einigen stellen, wo unser gelehrter diese bedeutung geltend macht, = 'vor' gericht auffordern (zum zweikampf)' (vgl. mnd. grôten 'zum kampf auffordern') oder dem richter praediciert = '(wegen eines vergehens) vor gericht laden': dal dae schelten,

1) Wegen der unsicheren quantität des $e$ s. unten s. v. kĕttich. 
deer bodtingh hâldet toe middey, eer unden (vor 9 uhr) bannes bigonnen habba schillet om dat strŷd (den gerichtlichen zweikampf), deer (wozu) ma aldeer grêta schil bi sonna opgongh W 391,15; als dyoe sonna [sonne] sighende is ..., so ne thoer (braucht) di frîu Fresa êfter dâm dis deys (nach 12 uhr an dem tage) withstaen (sich zu stellen), om dat hy eer grĕ $\breve{t}$ ne was W 391,21; datter êne landisgrêlmanne berde (gebührte) ti byriòchtene ende di grêlane, dat yemma dat al riûchtelike grête ende riûchte $\mathrm{S} \mathrm{488,26.} \mathrm{Vgl}$. auch in bezug auf die eine und die andre bedeutung das (von v. R. ebenfalls unrichtig als 'verklagen' gefasste) compositum bigrêta: sidset hiă (die sieben nachbarn) him (dem zum schwertkampf aufgeforderten) so graet gued, da hi mit strîde bigr $\breve{e} t$ nârd (als man sich mit der auffordcrung zum schwertkampf an ilın richtete), soe schil hi dat mâra strîd oenghaen W 393,13 ; Jef ma him deer mitta stryd [striid] bigr $\breve{e} l$, so schil hi sprecka ete. W 394,381); ende ênes landes greetman berre ti bigretlane ${ }^{2}$ ief ty riachtane, dal iemma dat riúchtelika riûchte ende bigrêle $\mathrm{S} 488,30$; dae greethman ... et bigrête ende biriúchte S 500,13.

Gryowa 'einen einschnitt machen, schneiden', *griôde 'narbe'. S. s. 356.

Grôya, blôya, wâya. Die verba pura hatten im vorfries. (dem ags. zrówan, máwan etc. entsprechend) $w$ zwischen wurzelvocal und endung. Dies ergibt sich erstens aus *fên(i)sa, dem prototypus zu fliues- in fliueswerp (s. 8. v.); zweitens aus den fưr wâyen $\mathrm{J} 59,18$, bivâiane $\mathrm{H} 149$ (d. b. wâi-jen, -jane) anzusetzenden prototypus ${ }^{*} n \hat{a}-j a n e$, dessen nicht zu $\hat{e}$ afficierter vocal auf altes *wâwan etc. schliessen lässt mit rlicksicht auf die tatsache, dass $\hat{a}$ (aus germ. $\hat{a}^{e}$ ) vor $w$ erhalten blieb (vgl. die den s. v. fliueswcrp und trouwaden besprochenen formen blau, clau, pau(e)s, taune 'geräte' zu grunde liegenden vorstufen *blânes, -e etc., *clâwa, *pânes, *gitâna). (Fir das aofr. ist groninge mit on $=o u-n$ aus *grôninga zu beachten). Demnach wären als die regelrechten entsprechungen von ags. *zrówed, blówed, wáneঠ awfr. *grêt (= aofr. grêth, Gramm. § $275 \beta$ ),

1) In der nämlichen bedeutung findet sich das verbum in einem aofr. texte: to tha mâra strîde hebbe ik iù begrêt, Huns. 341, 20.

2) Vyl. das zu k ěttich bemerkte. 
*blêt, *wêt aus *grêwith, *blêwith, *wêwith zu erwarten; und wirklich finden sich in unsern quellen noch ein paar indirecte spuren solcher formen in den analogiebildungen grê 'wachse' H 180 und wie 'wehe' H 180 (aus *grê-e, *nê-e für *grône, *wâwe; wegen des ie fur $\hat{e}$ s. oben s. 361 anm. 2). Als die normalen formen begegnen indessen *grôy $(e) \iota \mathrm{S} 491,5$. Sch 661 . $\mathrm{H} 304$, blôy $(e) t \mathrm{~S} 491,5$. Sch 661 , wây $(e) t$, waeyt Sch 661. H 304. Ag 135 aus *grôjith, *blôjith, *wâjith, die durch die bekannte synkope des $n$ vor $i$ aus *grôwith, *blôwith, *wâwith (mit hergestelltem $\hat{o}$ und $\hat{a}$ und nicht synkopiertem $-i$-) hervorgiengen. Dazu als analogiebildungen grôye $\mathrm{J} 58,39$, wâyen, -ane etc. $\left.{ }^{1}\right)$

$\mathrm{Zu}$ wâya steht ein praet. opt. wâyde $\mathrm{H} 67^{2}$ ) und wâyd W 440, 17. 26. 441, 26 (mit apokope der endung, wie dêd, deeld etc., 8. unten zu quae(n)); zu grôya, blôya sind keine praeteritalbildungen belegt, doch vgl. man nwfr. groeide, groeit, bloeide, bloeit (mit als $u a i$ gesprochenem oei aus *ôi). Beim ersten blick möchte man vielleicht diese formen als durch anlehnung an die schwache flexion 1. klasse entwickelte neubildungen gelten lassen. Doch ist hier zu erwägen, dass die $w$-synkope jungeren datums ist als die oben zu biliouwa (causativ) erörterte entstehung von $w$ aus $v$ und dass letztere erscheinung erst stattfand, nachdem das postconsonantische $j$ der endung geschwunden war (s. zu trouwaden und oben s. 392 und 353). Zu der zeit wo grôya etc. aufkamen, gab es also keine schwachen verba 1. klasse mehr, die mit $j$ im anlaut der endung die aufnahme von grôya etc. in ihre klasse hätten veranlassen können. Hingegen wurde ein solcher anlass wol geboten von seiten der schwachen verba 2 . klasse, als dieselben nämlich ihr altes -ia (d. b. -ija) in -ja gewandelt hatten. Demnach können wir kaum umhin, als die allein möglichen schwachen neubildungen zu grôya etc. *grôade, *grôad etc. (nach -ja, -ade, -ad 2. klasse) anzuerkennen. Aus diesen aber konnten dann in der folge, wie uns *layede und layed (statt ladede, ladet

1) Die Beitr. 17, 316 vorgetragene auffassung von grôia etc. aus *grôde, *grôd etc. ist zu verwerfen, weil die übereinstimmung im $w$-typus uns nötigt, analoge erhaltung der starken flexion im vorfries. vorauszusetzen.

2) Hiernach ist das $\mathrm{H} 69$ stehende, mit stoede opt. praet. verbundene wâye in wâyde zu corrigieren. 
durch anlehnung an laya aus ladia), layde und layd lehren (s. zu flet), zunächst *grôyade, -ede, *grôyad, -ed etc., darauf *grôyde, *grôyd etc. hervorgehen. (Eine parallele zu gedachtem ubertritt gewährt das zu nîa 'weiben' (d. b. wîja) gehörende p. p. wîad, -de H 105. 107. 108 neben urspr. wî(e)de(n), -da W 401, 22. 406, 10.16. 407, 16).

In bezug auf den durch das oy der uberlieferten formen repräsentierten lautwert folgendes. Aus $\hat{o}-j$ musste nach dem oben s. 347 bemerkten auf lautgesetzlichem wege $o i-j$ entstehen. Doch weisen nwfr. groeije, bloeije sowie roeije 'rudern', floeije mit als $\hat{u} a i-j$ gesprochenem $o e i-j^{1}$ ) darauf hin, dass im praes. unsrer verba in folge von anlehnung an die schwachen praeterita das $\dot{o}$-element des diphthongs durch den laut von *grôade verdrängt wurde zu der zeit, wo derselbe noch monophthongisch war, oder zu der zeit, wo sich die $\hat{o}^{u}$-qualität schon entwickelt batte. Dass auch fừ wâya der nämliche vorgang anzunehmen ist, erfolgt aus der nwfr. aussprache wâi-je.

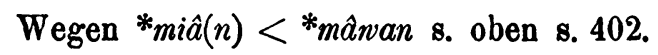

Hâna, behânet, behâniget. Wenn der zum gerichtlichen zweikawpf aufgeforderte als exception seine angehörigkeit zu einem andern gerichtsbezirk aufwirft, soll er nach dem in W $395-396$ und $\mathrm{H} 46-47$ ( $\$ 47$ und 48) beschriebenen verfahren solches beweisen durch die aussage des 'schelta', der in dem von ihm vindicierten bezirk amtiert, oder, wenn letzterer diese aussage verweigert, durch einen eid. Ferner soll

1) Awfriesischem, das nwfr. î̀ vorbereitendem $\hat{e ̂}^{i} \partial$ aus $\hat{e}$ (s. oben s. 361 ) entspricht ein das nwfr. (durch die schreibung oe dargestellte) uo vorbereitendes $\hat{o}^{u} \partial$ aus $\hat{o}$. Vgl. die neben der normalen schreibung 0 oder oc manchmal begegnenden ue und $u(u)$ : bueck 'buch' $W 433,9.434$, 14. 438, 28. J 17, 2. 50,30. 56,3.12.81, 7, bucket (bueket) 'der kirche vermacht' (eig. 'buchen lässt', vgl. Wb. i. v. bôkia) J 46, 20, dueck, duc 'tuch' $\mathrm{W} 476,6$, $\mathrm{H}$ 154, huede custodia W 437, 27. J 86, 2, huede servet W 410, 5 , hudit [hudel] custodit $\mathrm{J} 60,2$, buet(h), buete 'busse, zauberei' $\mathrm{J} 1,29.2$, 28. 80, 5 (vgl. wegen letzterer bedeutung mnl. boete 'zauberei', Mnl. wb. 1,1345), wrhuer, -huur 'ehebruch' J 9, 5. 47, 7. 12. 84, 21. 85, 1. 86, 13, huer-, huurkîynd J 47,11, wrhuerd moechatur J 85, 4, blued J 50, 24. 52,1. 56, 1 (Hett. falsch bloed), fuetloes $\mathrm{J} 66$, 2 etc.; beim reflex von altem gôd subst. und adj. ist sogar ue häufiger als oe: gued, guet passim in W, S, H und $J$, neben goed, goet $W 428,16.434,27.28 .440,29$. H passim. J 47, 9. 65,11 . 
er zwei seiner nachbarn zur verfugung haben, welche hine burgie, ende datse alsoe rŷck sê, dat hy $\vec{a}$ dine strŷdwirdiga schet (die entschädigungssumme, welche den zweikampf veranlasst hat) al lassta moghe, ief him di schelta hâna lêth, dal (sodass) hi aldeer (in dem bezirk, wo er aufgefordert ist) anderda (sich verantworten) schil. Was ist mit diesem hâna lêth gemeint? $\left.{ }^{1}\right)$

Hâna erscheint in den aofr. quellen (s. das Wb.) als 'kläger' und 'beklagter' (eig. 'der eine rechtskränkung erfahren bez. vertibt hat', vgl. mnd. hôn 'rechtskränkung'); in den awfr. als 'der geschädigte' oder 'der beleidigte, geschmähte' (vgl. mnd. hôn, mnl. hone, hoen 'schmach, schimpf'): Een riûchter, deer wytlick...f fynt een falschen doem ..., dîy is eerloes ende is da hâna schyldich op to rialchlen al ... sŷn schade $\mathrm{J} 18,18$; Hweerso een man sŷn gued wirlh bynymen mit macht off sŷn gued wirth schada deen, so aegh dij hâna da wirden fan da gued... to wirden $\mathrm{J} 54,6$; Hwaso ôrem schândelyck off lasterlyck wirden (worte) spreckt, deer in sŷn êra gaed,... so mey hij qwaen (sagen), dŷr (zudem) dat lasterlyckke wird spritzen is: Dit is meij (lies mij) alzo leed, als $X$ merck fan mŷn ayna gued jeffta meer, ... ende alzo fula [fule] so dij (dem) hâna to der hând to wysen ende to riûchten $\mathrm{J} 63,2$; . noch $\mathrm{J} 59,12.62,9$. 16. $65,18$.

In keiner dieser bedeutungen wäre das wort in dem oben zuerst citierten sat\% erklärlich, und es ist deshalb geboten, sich nach einer andern hierher passenden umzuseben. Bei diesem versuch aber bietet uns die im mnl. ubliche bedeutung von hone, hoen, nämlich 'betrug', eine willkommene hilfe; denn aus diesem hone, hoen ergibt sich die möglichkeit eines awfi. *hâne oder hân 'betrug', wozu hâna 'betrigger' das an der in rede stehenden stelle einen guten sinn ergeben durfte: wenn der schulze ihn (dem obwaltenden verdacht gemäss) betruger sein lässt ${ }^{2}$ ) (indem er ihn nicht als zu seinem bezirk gehörend anerkennt).

In dem oben verzeichneten hâna 'geschädigter' gehört als

1) จ. K.'s hana 'von hiınen' (Wb. 796) kann natürlich unbesprochen bleiben.

2) Wegen lêth 'lässt' mit th als schreibung für $t$ s. die citate im Wb. und vgl. unten. 
denominativ behâniget 'geschädigt, ubervorteilt' in weert seeck dat wedue off weesken off tzercke wêre behaniget off byclôket (vgl. oben s. v.) myt da ferdban, so is dij riachter ... dat ferdban wanmachtich to rîchten $\mathrm{J} 31,10$; Hweerso dy $\overrightarrow{0}$ tzerck of gâstlicke lyoed off wêsen sint behâniget ..., dal aegh neen slal $J 32,15$. Metaphorisch von einer sache verwant steht das verb. = 'beschädigen' in weer dat huus wtvârdis byhânet my (lies myt) ênigherhânda tingh, dat to bêten myt een hâla pond $\mathbf{J} \mathbf{7 5}$, 3. Wegen der suffixe -(i)gia, -egia etc. fur -ija etc. bei verben der 2. klasse (= aofr. -igia etc., Gramm. § 299 anm. 1) beachte:

eynd(i)gie, -get W 438,31. H 29. J 19,5. Sch 735, endeghia $\mathrm{H} 150^{1}$ ) (nwfr. eindigje, eyngje und eynje GJ, aofr. endigia etc. neben endia, -ath = ahd. entôn); crywsgade p. p. 'ein kreuz tragende' J 20, 8 (nwfr. krjuesgje 'quälen' GJ, = ahd. crûzôn); pŷnigien, -igie, -egat S 485,16. J 62,8. H 22, pyngia, -gien, -gie, -gat, -get S 459,5. J 2,9.33. 12, 22. 15, 18. 23, 5. 10.11. 43, 7. 65, 1. 5, pinnigia, -iget W, J 20, 7 ') (nwfr. pijnnigje GJ, aofr. pînegat F 4 = ahd. pînôn); foerlènighet 'verliehen' J 60, 19 (nwfr. forlienge p. p. neben forlienje inf. GJ, aofr. lêngade = ahd. lêhinôn); byhyndiget 'beschimpft' (s. unten zu hyndicheed); needmondighiet, -iget J 86, 8. 66, 5 ( $\mathrm{uu}$ nêdmond 'notzucht'); biriousiget 'bereut' $\mathrm{J} 86,10$ (= ahd. riuwosôn, ags. hréonsian); bistedigia 'bestatten' W $\mathrm{W}^{1}$ ) (= mul. mnd. besteden 'bestatten' aus *bisled(i)ôn); *laun(i)gia 'laben' und *klins(i)gia 'reinigen' (s. oben s. 354 und 369);

sowie oneerwighia 'enterben' H 173 neben (ont)e(e)rwia,

1) Spuren des ulten -(i)g- (vgl. Aofr. gr. 8. 234) begegnen noch in eyndiget 3. pl. J 15, 71, pînigha, -ghen S 484, 24. J 1, 36. 29, 1. 38, 4. 62, 1, bestedig in J 34,1, schouwiga (8. 8.400). Aus der periode, wo formen auf dieses -(i)g- mit unursprïnglichen, später normalisierten auf -(i)gi- wechselten, stammen die als analogiebildungen $z$ fassenden, zu denominativen aus igadjectiven gehörenden formen (denen von haus aus nur -igi- zukam) bysihyldiga J 12, 15 (neben on(t)schyldigia, -igie J 6,1. 39, 2. 62,9), wŷtiga 'weissagen' $\mathrm{J} 80,6$ (neben wŷylgien $\mathrm{J} 80,8$ ), wrdiligen $\mathrm{J} 64,17$, foerdilga $\mathrm{J}$ 1,1 (neben ùrdilighied $W$ mit kurzem wurzelvocal, nicht mit $\hat{\imath}, \nabla g l$. mnl. deligen, dessen $e$ nur auf $i$ zurückgehen kann), sowie swîget 3. pl. J 36, 9 (2 mal, neben snîgia, -ie etc. W. H and J passim). Umgekehrt veranlasste dieses -igia etc. neben -iga mitunter die verwendung eines -igiet fiir -iget der 3. sg. praes. und des p. p.: aefligiet, law(i)giet(h) 3. 8g. und p. p. J $47,3.6 .50,1$. 30. 41 . 
-ien W, J 45, 1. 11. 33. 46, 58. 51, 2. 58, 16. 77,6. 84, 18 (nẃfr. eervje GJ, aofr. erwie, -iat etc.); launigia, lauwegia, -eget, laugia, -gien etc., lawigia etc. 'nachlassen' W, H und J passim (s. auch oben s. 354. 363) und lawie, lauvede, lawede $\mathrm{W} 105,13.73,31$. 398, 36 (aofr. lâvegad, lângat und lâwiane, Gramm. s. 231. 234); wtreysget 'zum kampf auszieht' $J$ 72, 5 (nwfr. reysgje 'reisen' GJ) und reysia, -ien 'reisen, ziehen' Sch 546. 600. 601. 609. 655. 733. 739 (ahd. reisôn); wenghien 'wohnend' Ag 107 und wennia (s. unten s. v., nwfr. wenje); schouwiga ${ }^{1}$ ) Sch 545 und scouwia etc. (s. oben s. 377 , nwfr. oonschounje GJ), sennigia 'streiten', sennegat H 61. 142 und sannia W 411,20; schadigia, -gie, -get W, J 37, 4. 38, 2 und scha(d)ia, -et(h) W, H 34. 117. J 26, 13. 30, 4 (= ags. sceadian). S. noch zu trouwaden.

Hatâ, hettâ 'etwas' in Beyda igghen (parteien) habbel instrumenta jeffta breef [brief], deer se mey willet hatâ bivîsa

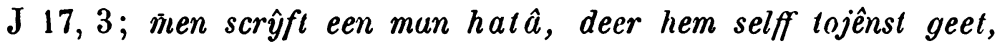
dat scrift steet wel to lyowen (glauben) $\mathrm{J} 17,15$; Hwaso eenich [ênich] dêde [dêda] schyldich is jeffta een ôrem hat $\hat{a}$ jaen jeffta dwaen schôlde J 38,4; Dat dij fader da kŷnden naet lêgia wôlde wter fanscip (lies fangenscip) jeff da kŷnden bûla wirth wôrden jeff jelkers [jelkirs] hat $\hat{a}$ tocoem $\mathrm{J} \mathrm{51,2;}$ men wilma him hetl $\hat{a}$ jaen (geben), dal mey hij nima J 77, 9.

Der auslautende vocal des pronomens kann schwerlich ctivas andres sein als das dem indefinitum angehängte adverb $\grave{a}$ 'immer' (wegen dieses wortes s. H 83. 179. 180. J 27, 1. 15, 55 ; wegen der nämlichen verwendung von $\hat{a}$ in enter $\hat{a}$ etc. und im aofr. aiderâ und ahwederâ s. oben s. v. ent(h)er â und Aofr. gr. § 257 anm. und 269). Hinsichtlich des vocals und der consonanz von hu|l- und hell- vergleiche das normale hael (ali)quid, quodcunque $\mathrm{W} 27,31.398,32.424,14$ (bei v. R. steht hier unrichtig hwael). 434, 26. 27. 29. 435, 1. 5. 23. 440, 7. 474,16. H und $\mathrm{J}$ passim mit tonlangem $a$ aus ${ }^{*} h(w) a t a$ (= got. *hwata, vgl. aofi. hwete, Gramm. $\$ 252$ und s. Beitr. 15, $474 \mathrm{ff}$.) und het quodcunque $J$ 44, 8. 71, 6. 75, 9 (nwfr. het GJ) mit $e$ in geschlossener silbe für $a$ aus *h(w)at. Für den verlust des alten keinesfalls auf phonetischem wege geschwundenen $w$ ist beeinflussung von seiten der doppelform hot $\mathrm{H}$ 1. 73. 175300.

1) S. anm. auf s. 399 . 
301. 302. 303. S 498,21. Sch 394 (mit $o$ aus $w a$ ) geltend zu machen. (In dem seltenen hwat $\mathrm{H} 150^{1}$ ) und den im jetrigen $\mathrm{nwfr}$. tiblichen formen hwat, hwet ist das $w$ wol nach dem muster der andern flexionsformen hwa, hnaem etc. hergestellt).

Haetia. Im $\S 19$ des Rudolphbuches (W 431 und 432. $H$ 171), wo eine reihe von handlungen erwähnt wird, welche die öffentliche sicherbeit gefährden und deshalb ausdrücklich verboten werden, heisst es u. a. Ick wrbanne, datter nemmen haetie da heerstrêta ...; hwaso hŷr wâld oen dêt, dî schilt bêta mit een coelscha pond. v. R. ubersetzt hier haetie durch 'ziehe' (Wb. 798), was sich weder in bezug auf die form noch hinsichtlich des inhalts des paragraphen rechtfertigen lässt. Den weg zur richtigen deutung zeigt uns das mnl., wo haten in verbindung mit einem ohject wege, dijck und dgl. als 'beschädigen' gilt (s. Mnl. wb. i. v.), begreiflicherweise in folge ciner metaphorischen ubertragung des ursprünglich mit einem acc. personae in der bedeutung 'anfeinden' verwanten verbums (vgl. ags. hatian, as. haton infestare aliquem).

In J 56, 6 stebt hatien 'hassend'; vgl. nwfi. haetsje 'odisse'. Haudstoe und haudstoed 'bauptkirche'. S. zu stwê.

Hensich (hinsich) ende bêrich. Das adject. hêrich und die formel hensich (hinsich) end(e) hêrich, die ursprünglich = 'untertan (und unterworfen)' in bezug auf personen verwant wurden (s. das Wb. i. v. heroch, sowie W 11, 14. J 50,48 2), und vgl. das ijbereinstimmende aofr. hanzioch and hêroch, hendsegch, -zeg and (end) hêregch, Aofr. gr. § 27 R1. 139. $143 \beta$. $35 \alpha$ ), begegnen in den awfr. quellen auch in verbindung mit sîl 'schleuse', sîlrôde 'mit einer' schleuse in verbindung stehender graben', dŷcken, dammen, wettringe, weghen, lanen (mit bäunen bepflanzte wcge): Foerd soo bifella wyt alle dac ienne, deer habbit zŷlen ieflu zŷlroeden, dîken, weghen iefla dammen, dalse (lies dutse se) tyaen ende têmen (uber diese part. praes. gleich unten), hensich ende hêrich hâlda $\mathrm{S} 491,30$; ende diŏ mêne

1) Ob in diesem hwat und in hat (hath, hadt) W 474,30, H 299. 302 das $a$ schreibung ist fuir tonlanges $a$ oder kurzes $a$ darstellt, ist $\mathbf{z w e i f e l -}$ haft. Fiir die möglichkeit des letzteren sprechen die bei GJ 202 verzeichneten formen hat, het und die jetzigen $\mathrm{nwfr}$. hnat, hwet.

2) Wo die wörter in umgekehrter folge als hêrich ende henzich (hinzich) stehen. 
meente den dŷck hêrich to meythien (zu machen) Sch 545; ende dae weghen iefta lanen ... hinsich ende hêrich toe halden Sch 603; on toe bysjaen dan den Nŷ-sŷl, ofter hensich en hêrich is, ende is der naet hêrich, dan toe bysjaen een netten leger Sch 714; dat Audamirdera . . schillet den Nŷsŷl lidza ende hêrich mekkia ... ende dattir hensich en hêrich zee. by Sunte Andreas dey nêstkommen Sch 717; dat Sindebra... schillel den Nŷ-sŷl etha lidza ende hensich en hêrich mekkja ibid.; dat da dŷcken, dammen, sŷlen ende wettringe hensigh ende hêrigh macket wirdit Sch 732.

Die nicht zu verkennende bedeutung der adjectiva 'zur dienstleistung geeignet' ist selbstredend die folge von metaphorischer libertragung der ursprunglichen bedeutung ' $z u r$ dienstleistung bereit'.

Statt hensich ende hêrich erscheint als prädicat zu sîl, dŷyk etc. auch tiaende ende temende: dyne sŷl tyaende ende thmende habba und die andern im Wb. i. v. tema zu findenden belegstellen sowie das oben verzeichnete citat aus $S 491$, wo t. e. $t$. neben hens. e. hêr. stehen. Hieraus geht hervor, dass die beiden formeln gleichbedeutende oder doch ungefähr gleichbedeutende waren, dass also v. R.'s fassung des tiaende als '(wasser) ziehend' und des $t \vec{e}$ mende als 'zähmend (zur'tickhaltend)' oder 'vacuum reddens' a limine abzulehnen sind (zu dem object dîken etc. in $\mathrm{S} 491$ würden diese bedeutungen ohnehin gar wenig passen). Fùr têmende ist wol nicht mit unrecht an ahd. gizami, got. *gatêms (vgl. gatêmiba) anzukntipfen, mithin ein denominatives * têma für *gitêmian = 'im gehörigen zustande sich befinden' anzunehmen (wegen des schwunds von gi- 8 . oben zu beithe). Fur tiaende, das naturlich nicht zu tiaen 'ziehen' gehören kann, liegt es nahe, un ein derivatum zu *lêni $=$ 'in ordnung' zu denken (vgl. aofr. eltê 'gesund', Aofr. gr. $\S 86 \alpha$, ags. cellóene 'vollkommen' und got. têwa $\tau \dot{\alpha} \gamma \mu \alpha$, ungalêwidai

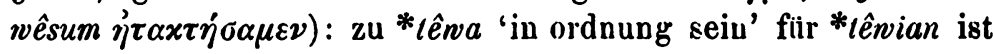
als 3. sg. praes. ind. *têth a us *tênith oder mit hergestelltem suffix * aus diesem *lêth oder *lế-eth aber konnte sich als neubildung ein inf. *têa(n) entwickeln, der in der folge zu *tiâ(n) werden musste (vgl. nwfr. mjean 'mähen' $=$ awfr. ${ }^{*}$ miâ( $(n)$ aus *mên $(n)$ statt ${ }_{m} \hat{a} w a(n)$ nach der 3. sg. praes. ind. ${ }^{*} \hat{e} t h=$ aofr. $m e \hat{l} l h$, 
Gramm. $\$ 274 \delta$, oder $*^{*}$ ê-eth $\left.<*_{\text {menvith }}{ }^{1}\right)$ und beachte noch wegen $i \hat{a}$ aus $i a$ aus $\grave{e}-a(o)$ awfr. auf *niâr $=$ aofr. niâr, Gramm. $\S 16$, zurtickgehendes niêr, niâr 'näher' 2) aus *níar aus *nîar aus nêar aus nêhor $=$ as. nâhor).

Einmal begegnet statt der erwähnten formeln rekenad ende têmed in $1 c$ banne ioe hêren alle meenlike, dat $i$ iouwe insîlen ... also rekenad ende têmed habbe ti der sêburch, dat se onbanplichtich sê H 119. Letzteres p. p. gehört augenscheinlich $\mathrm{zu}$ einem trans. *têma = 'in den gebiihrenden zustand bringen'. Das andre begreift sich bei der annahme eines $*$ rekenia $=$ ahd. rechenôn parare, denominativ zu aofr. rekon, ags. recen paratus.

Herde 'arbeit'. S. zu wt ha fussn.

Hêrmscheed. Das subst. steht als synonymum zu hêrmscere = 'auferlegte busse'. Das im Wb. herangezogene ags. scéat portio muss selbstrerständlich aus dem spiel bleiben. Für die deutung von -scheed ist mnl. gesceet 'urteil' (Mnl. wb. 2, 1594) $\mathrm{zu}$ beachten; also hêrmscheed = 'verurteilung zur busse' aus *hêrmgiscêd (wegen des schwunds des gi-s. oben zu beithe). Hêrstet in Deer lauwa (den glauben) breckt ende deer tojênst dwaet ende herstet $\mathrm{J} \mathrm{81,23.} \mathrm{Als} \mathrm{die} \mathrm{bedeutung} \mathrm{dieses}$ hêrslia ist 'blasphemieren' anzusetzen. Ich bringe das verbum in verbindung mit an. herst = 'in gereiztem ton' und erinnere an das verbāltnis von ag. hier(w)an 'blasphemieren' zu mbd. here, hare (flect. herwer, harwer) 'berb, bitter'.

Hetene in diöleste hetene, deer da kempen dis tredda deys fiûchtet, dyô haet diŏ lâckhetene W 394, 22. Das wort ist

1) Wegen des $v$ von "mâwan 8. zu grôy a. Ob *mêth, spîl und *lêth durch synkope des $w$ vor $i$ oder durch schwund des endungsvocals und nachherige absorption des für $w$ eingetretenen $u$, ev. nachherige synkope des $w$ (wenn dieses schon spirans geworden war) entstanden, lässt sich schwer entscheiden. Analogischer ausfall des - $i$ - war schon im auf kommen, als das alte $\chi$ noch nicht zum hauchlaut geworden war (Beitr. 17,557), ist mithin eine ziemlich alte erscheinung. Hingegen ist der ausfall von $w$ vor $i$ nach rêsien (8. unten s. v.), stoya, scoya etc. (s. unten zu trouwaden) jüngeren datums als die genesis von - $i$ - aus -a(für -o- aus - $\hat{o}^{-}$) im stammsuffix der schwachen verba 2 . klasse und die entwickelung des $w$ aus $v(i)$ gehört also einer verhältnismässig jungen periode an.

9) Vgl. das zu sauwen über awfr. iềa aus $i \hat{a}$ bemerkte. 
mit v. R. als 'gang im gerichtlichen zweikampf' zu fassen; was im Wb. liber seine etymologie bemerkt wird (zusammenhang mit engl. hit) befriedigt aber keineswegs. Die endung -ene (= got. -eins) weist auf ableitung aus einem $j a$-verbum hin (vgl. haudlêsne, grêlene, wapeldêpene, heftene, hendene etc.); die bedeutung des nomens führt auf den gedanken, got. hatjan heranzuziehen und wegen der begriffsentwickelung an. hetja 'kämpe', ags. hettend 'feind' (woneben auch helend durch anlehnung an hete) zu vergleichen.

Luckhetene ist schon im Wb. richtig als 'schlusskampf' erklärt.

Hyndicheed, -heet. Das nomen findet sich in Alle onnelte hyndicheed nêr laesheed ... can een man naet helpa, ende hwaso qwêlyck recknet jeff foertbringt, dy bycrîged nael

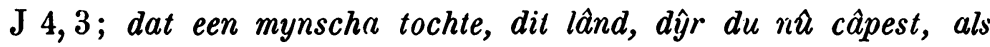
ick dat jeld hab, so mey ick hym weer oeffdrywe fan da caep off wandel myt nyaer (durch näherkauf), dat is onnette hyndicheet ende schyl hym schadya ende naet helpa $\mathrm{J}$ 30,4. Ein hierzu gehöriges adject. byhyndig begegnet in Hweerso een man falsck swert fara een ôrem ende dij ôra meent, dat hij him al riucht to fara [fare] swerth, als hijt him to fara leyt to sweren, ende dijjen, dŷr dan swaere [sware] schil, myt byhyndiger falsckheit oers swert ende meent, hij sê dan naet meeneendich (lies meeneedich), indien dat hij dan alzo swerl, so is hij meenêdich J 24, 14.

Für die deutung der wörter an verwantschaft mit mbd. mnd. mnl. behende zu denken, verbietet der umstand, dass altes $e$ vor $n+$ dental im awfr. zu $e i$ wird (s. oben zu biriind?). Hingegen berechtigt die oben unter onterâ beobachtete erscheinung, nämlich die entwickelung eines zwischen $e$ und $i$ liegenden kurzen vocals aus $\hat{e}$ vor $n+$ dental, hier einen prototypus mit $\hat{e}$ aus $\hat{o}$ anzunehmen, m. a. w. das adject. *hindig als derivatum $\mathrm{zu}$ altem *hênda oder $-u$ aus $*$ haunilhu $=$ ahd. hôn(i)da, as. hônda, ags. hienð(o) zu fassen. Zwar begegnen die letzteren formen nur in der bedeutung 'schimpf, schmach' bez. 'schaden', doch ist mit rücksicht auf das mnl. hone, hoen 'betrug' (vgl. auch oben zu hâna) die möglichkeit eines *henda oder $-u=$ 'betrug', bez. eines *hindig mit der an den citierten 
stellen völlig passenden bedeutung 'betrugerisch' nicht $\mathrm{zu}$ läugnen.

Dass *hênda indessen auch die bedeutungen 'schimpf' und 'schaden' zukamen, ergibt sich aus henda in thêr (der) um thes lôndis nilla sêrnesse ieffta henda hebba, Rechtsqu. 309,16, und aus dem denominativ byhyndiget 'beschimpft' in So scheller nymmen behyndiget nêr bylastereth wessa fan een oers quâda dêda J 64, 23 (wegen -ig- s. oben s. 399).

*Hiouwera 'hafer'. S. s. 359.

Hirda 'antreiben'. S. zu wtha fussn.

Hlackia. Das $c k$ dieses in den awfr. quellen nur im praet. hlackade $\mathrm{W}$ und $\mathrm{H} 63$ belegten verbums ist nicht nach Beitr. 17, 318 auf hh zurückzufübren, denn letztere consonanz hat dem nwfr. pochje GJ zufolge ihre spirantische qualität nicht aufgegeben. Man vergleiche hingegen an. hlakka, -aða, -at 'sich freuen, kreischen' (rom adler gesagt), eine form der kategorie ahd. zocchôn, ags. twiccian etc. (s. Beitr. 9,163 ff.). Auffallend ist jedoch das $a$, statt dessen man nach bec 'rucken', wrbec 'binterrücks, zurück' W, S, H 158. J 24, 2, ecker 'acker' $\mathrm{W}, \mathrm{H} 77$, leck vituperium $\mathrm{W}, \mathrm{S}, \mathrm{J} 65,13$ e erwarten musste. Durfte man hier vielleicht anlehnung gelten lassen an ein verwantes *hlahtor (= ags. hleahtor), in dem das a vor ch (vgl. bitrachtia $\mathrm{H} 304.305$, wachtien $\mathrm{J} 43,9$, nach interjection $\mathrm{W} 439$, 20. H 6, 4, macht, manslachta etc.) lautgesetzlich erhalten blieb, oder hat am ende ein altes *hlahh(i)a(n) die form des schwachen verbum beeinflusst?

Ueber die nwfr. reflexe von hlackia s. unten zu meyt(h)ia.

Hli âpa. Neben altem $(h) l u e p t(h)$ 'läuft' W, S, H 2l, hlâpet(h) $\mathrm{W}, \mathrm{H} 22$, mit p. p. hluepen $\mathrm{H} 88$, erscheint in den quellen auch eine praesensform mit iâ: hliaept $\mathrm{W} 435,15.25$ (nwfr. ljeappe GJ), deren analogische entwickelung sich erklärt aus dem zu hlâpa gebörenden praeteritum hliôp (mit $i \hat{o}$ in folge der nämlichen accentverschiebung, die aus alten $i u$, $i a$ die historischen $i \hat{u}$, iâ entstehen liess; für die belege $\mathrm{s}$. hlioep in dem von Hettema in $J$ nicht abgedruckten teil des ms. s. 7, hliôpe opt. S 494, 18. 497, 21): hliapa neben hliôp nach stapa mit tonlangem vocal (vgl. stapt $(h) \mathrm{S}$, stapet W und p. p. stapen J 15, 54) neben sloep W, H 114, fara neben foer, draga, -ene etc. W, 
S, H 56. 58. 104. 107. 300. 302. Sch 708. J i2, 4 1), neben droech. ${ }^{2}$ )

Von den andern reduplicierenden verben mit wurzellaut $\hat{a}$ (aus $a u$ ) und $\hat{o}$ sind, so viel mir bekannt, aus unsern denkmälern keine formen für das praet. zu belegen: von rôpa und ho(u)wa, ha(u)wa erscheinen ausser den praesensformen (s. fur das erste verb. W 35, 2. H 120. J passim, fur das andere oben s. v. fliueswerp) nur das p. p. rôpen J 15, 70. 18, 9. 25, 7. 21. 46, 45. 57, 11, ho(u)wen, ha(u)wen (s. zu fliueswerp) $)^{3}$ ); von blôya, grôya und wêpa 'wehklagen' begegnen nur blôy(e)t, grôy(e)t, grôye (s. oben s. v. grôya), wypt $\mathrm{H} 85^{4}$ ) (mit durch kürzung aus $\hat{e}$ entstandenem $i^{e}$, vgl. das zu enter $\hat{a}$ besprochene $i^{e}$ aus $*_{\hat{e}}$ vor $n n$ und $n+$ dental); reflexe von ags. béalan, ahd. scrôtan, bluozan etc. fehlen gänzlich. $\left.{ }^{5}\right)$ Doch ist wenigstens für rôpa noch ein praeter. *(h)riôp zu erschliessen aus $\mathrm{nwfir}$. zu

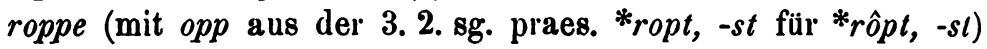
gebörenden rôp 'rief' (GJ und Bl 151) mit synkope des ersten componenten, wie in den oben 8. $351 \mathrm{anm}$. verzeichneten formen.

In betreff der chronologie der genesis des praeteritalem $i \hat{o}$ $\mathrm{zu}$ grundo liegenden $i o$, das entweder durch $e o$ oder durch $i-\hat{o}$ aus $e-\hat{o}$ hervorgieng, sei noch bemerkt, dass es jiingeren datums ist als das io der entwickelungsreihe germ. eu zu eo zu io zu ia zu ia zu bistor. abwechselnd dureh $i a(e)$ und ie dargestelltem $i \hat{e}^{a}$ (vgl. unten zu sauwen); denn wenn das praeteritale io oder event. dessen vorstufe eo mit dem io oder eo dieser reibe zusammengefallen wären, musste das praet. $i \hat{a}^{e}$ statt $i \hat{o}$ baben. ${ }^{6}$ )

Iauwelik, iêwelik 'jeder'. S. zu sauwen.

1) Doppelformen zu drega, -e, drecht W, H 89. Sch 649. J 32, 19. 37, 2, dregge, -en, -et $\mathrm{J} 13,45.37,4$. Sch 395.637.

2) Umgekehrt steht hloep H 28 a!s praet. statt hlioep durch anlehnung an hlâpa.

3) Das im Wb. als praet. verzeichnete rôp Jur. 2, 266 ist praes. opt.

4) An der parallelstelle in der incunabel $W 47,10$ steht verderbtes wyst.

5) Wegen gliaender $W 77,25$, H 95, das ich jetzt nicht mehr auf *glôwan zurückführen möchte, s. Beitr. 17, 315 f., spec. anm. 3 anf s. 316.

B) Weil das hier fiir das awfr. bemerkte auch fïr das aofr. zu gelten hat, ist das Aofr. gr. § 275 angesetzte ia in io zu ändern. 
Inka. In W 399, 7-9 werden als die bestandteile einer erbmasse erwähnt gôld ende weed ..., kû ende ey (schaf) ende hiâra quikfiâ ende ink a ende anderke. $\mathrm{Zu}$ inka bemerkt Grimm (s. v. R.'s Wb. s. 1164) 'abd. encho, anchio, mhd. enke 'ackerknecht', der das rind beim pflug lenkt, er wird neben kuh, rind und pfluggerät zum bodel gerechnet'. Mit recht bemerkt v. R. (a. a. o.) in bezug bierauf: 'der ackerknecht will mir unter gegenständen des bodel nicht gefallen, auch heisst anderke nicht gerade pfluggerät'. Das wort ist mit rúcksicht auf das folgende anderke 'werkzeuge, geräte' (plur. zu *anderk $=$ mhd. antwerc ' werkzeug') 1) als bezeichnung für 'ackergeräte' zu fassen und muss der plur. sein zu einem neutr. $i a$ stamm *ink (e) 'ackergeräte' = ald. *anchi, enchi, wozu als denominative an-bildung anchio, encho 'ackerknecht' (vgl. got. spilla zu spill, ags. stéora zu stéor etc., Kluge, Nomin. stammbildung $\S 16$ ).

An der parallelstelle in $\mathrm{H} 58$ steht emka als ein durch falsche auflösung von $\bar{e} k a$ entstandener copistenfehler. Wegen der auf eine zwischen $e$ und $i$ liegende vocalqualität hinweisenden wechselsehreibungen $e$ und $i$ zur bezeichnurg des durch $i$-unlaut aus $a$ bervorgegangenen, vor gutturalem nasal stehenden lautes vgl. bringa, -ane etc. W, S, H 48. 52. 147. J passim neben brenga, -ane ete. W, S, H passim. J 1, 10. 13, 29.

1) Wegen der synkope des $w$ in unbetonter silbe nach dental s. ausser anderk $\mathrm{H} 58$, onderk $\mathrm{W} 422,34$ noch anderda, -e 'sich verantworten' S, W 13,18. 6i, 18. 73,36. 395, 16. 396, 15. 401, 10. 407, 14. 408, 5 . 419,4 . 27. H 41. 54. 62. 92 etc., J passim, andera (mit $r$ nach tonlosem vocal für $r r$ aus $r d$ ) $\mathrm{W} 73,2 i$ mit andert 3. sg. praes. ind. und p. p. J passim, anderda 'iiberantworten' $\mathrm{W} 69,1.395,1$. H 47. 115 mit anderl 3. sg. praes. ind. und p. p. W 417, 34. 37, anderl 'antwort, verantwortung' $\mathrm{W}, \mathrm{S}, \mathrm{H}$ und J passim, andert 'gegenwart' $\mathrm{W}, \mathrm{S}, \mathrm{H}$ und $\mathrm{J}$ passim, anderdia 'sich verantworten' $H$ 86. 89. 94. 109. 110. 122. 131. 134, and(e)riane, -ien, -ie W, S, Sch 535. H 40. J 1, 14. 7, 7. 10. 8, 2. y. 10. 10, 2. 20, 1. 32, 24. 37, 13. 46, 66. 59, 13. 62, 4. 67, 3, wrandria J 8, 9. 10. 25, 11. 17. 35, anderdia, -aden, -at 'überantworten' H 48. 131. 132, wide 'witwe' W 19, 21. 63, 30. 426, 37, H 32. 71. 121. 161. Daneben andwirk $\mathrm{H}$ 143, andwert 'antwort' J 3, 6, andwirde 'verantwortung' H 120, antwirdie (antw'dia) 'sich verantworten' $\mathrm{J} 67,6$, andwerdia, -ie 'iiberantworten' $\mathrm{H} 92$ (Hett. falsch

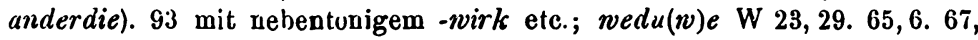
17. H 91. 92, J 11, 6. 30, 8. 9. 31, 10. 50, 39. 41. 69, 1. 87, 4. Vgl. für dieselve erscheinung im aofr. Gramm. $\S 85 \gamma$. 
15, 1. 83,1, oenbringh, -brins, -brinsche etc. W, S, H 150. J 13, 39. 30, 30, opbringh J 8, 8 neben oenbreng, -brensze, -brencsze etc. W, S, H 88. 136. 139. J 13, 21, hingia 'hängen' W, J 1, 43. 80, 2 neben henget Sch 604, hinzia 'gutbeissen' W neben henzia H 21, bit(h)inzia 'bedenken' H 60. 178, tynsa, -za 'denken' J $21,18.28,7.33,7.64,17.65,14$ neben bitensa W, hwinsen, -(d)zen 'gehangen' W, Sch 389. 462. 516. 517. 522. 530. 608, hinsen Sch 616 neben hwensen, $-(t)$ zen Sch 495. 540.616. Ag 6. 56, hinghnisse $\mathrm{W}$ neben henghnese, -nisse $\mathrm{W}, \mathrm{H}$ 108. 159, hinsich neben hensich (8. oben 8. v.), tohinsinge $\mathrm{J} 60,3$, finsen, -zen 'gefangen' W, H 143. Sch 590. J passim neben fens(z)en, -zen W 395, 21. Sch 250. 389. H passim, ginsen, -zen W, H 169. $\mathrm{J}$ passim neben gensen, $-z(i)$ en $\mathrm{W}, \mathrm{H}$ 118. 124. 138. 162. 164 etc., ginse 'gangbar' W, singht 'sengt' W neben senghet $\mathrm{S}$, sengt $\mathrm{H} 90$, svârt(a)svingh perfusio W, H 145 neben swêrtasweng $\mathrm{W}$, $\mathrm{H} 92.111 .145$ etc.

Jow n 'gleich'. S. 8. 358.

Jouwigh, jouwelik, ioegh 'ewig'. S. oben 8. 379 .

Jouwelik 'jeder'. S. zu sauwen.

$\mathrm{K}$ èttich 'bekannt' in $\mathrm{S} 502,20$. J 15, 32. 61. Sch 552. Die bei regelrechter entwickelung zu erwartende form wäre natürlich *kêdich aus *kêthich. Das $t t$ statt $d$ erklärt sich jedoch unschwer als die folge der beeinflussung von seiten des p. p. zu kêda aus *kêtha 'bekannt machen', nämlich $k \breve{e} t \mathrm{~W} 404$, 20. S 501, 4. 40 ${ }^{1}$ ), flect. k $\vec{e} t t e \mathrm{H} 102$ mit $t l$ aus stimmlosem thth aus stimmlosem $t h+$ durch vocalsynkope hiermit zusammengestossenem und assimiliertem $d^{2}$ ), wie in den praeteriten $k \vec{e} t t e \mathrm{~W}, \mathrm{~S}, \mathrm{Il} 25$, schaete $\mathrm{W}, \mathrm{H} 27$, schaet (-e apokopiert) J

1) Zwar findet sich daneben nicht selten $k \breve{e}$ th $W 41,23.388,18.20$. S 500,31. 505, 12. H 30. 83. 102. J 2,23. 3,13. 18, 10. 15; doch spricht diese offenbar als reminiscenz an die frühere orthographie haften gebliebene schreibung ebenso wenig für spirantische aussprache des dentals, wie z. b. das th in den participien seth 'gesetzt' W 23, 12. 45, 12. 418 , 24. 424 , 16. 425 , 19. 432, 1. 433, 15. 435, 13. 437, 19. J 21, 26. 28,16 , grěth W 412, 20 (zu grèta), beleth 'mit arrest belegt' J 20,17 (vgl. mud. beletten 'mit arrest belegen') mit th als schreibung fiir $t$ nach dem muster des mit $t$ gesprochenen $k \breve{e} t h$.

2) In betreff des stimmlosen thth aus stimmbaftem thth vgl. das $t$ t aus $d d$ in den praeteriten nêle und lalte (s. oben im texte), hlëtle 'lïutcte' W 410,9. H 114 (zu *hlêda), sante, wanten, seinte etc. (s. oben 
1,46 mit $t$ nach langem vocal als schreibung für $t t$ (zu skêda aus * skêtha), biněe tten 'setzten sich f(ir etwas der gefahr aus' $\mathrm{W} 440,19$ 1) (zu *binêda aus *binêtha; wegen dieses verbums vgl. Beitr. 14, 265 f.) und im p. p. schaet $\mathbf{J}$ passim, flectiert byschâlte, $-a$, onbescâlte (s. unten die fussnote $z$ u wrichte 'arbeiter'). In betreff des $t l$ aus thth vgl. noch awfr. smitte 'schmiede' W, H 143. 179. 180, witte 'band' W (witthe $\mathrm{H} 67$ mit $t h$ als rest der alten orthographie), swette 'grenze' Sch 547, atta 'richter' W, S, H 129. 130. 140. 150. 151. 153. 302 (altha H 154. W, S, Sch, J 24, 21, atha W mit alter schreibung) neben aofr. smithe (schreibung für smiththe), withthe, sveththe, aththa. (Aofr. gr. § $123 \beta$ ).

Bezüglich der quantität des $e$ in ket, kette, binetten mag ich keine entscheidung treffen: einerseits möchte man kürze des vocals annehmen mit rücksicht auf das neben normalen grêta, -ane etc. einmal begegnende bigrettane $\mathrm{S} 488,30$ und die nwfr. praeteritalformen (Bl. 144) blette, blet, fette op, opfet, lette, let, mette, met etc. zu bliede 'bluten', opfiede 'erziehen', liede 'lauten', mjitte 'begegnen' etc. (awfi. blêda, fêda, *hlêda, mêta), gegentiber ae (d. b. $\hat{a}, \mathrm{Bl} 143$ ) in laette, laet, skaette, skaet, spraelte, spraet etc. zu liede 'leiten', skiede 'scheiden', spriede 'spreiten' etc. (awfr. lếda mit praet. lâtte W, H 54. 81. 114. 134. 137. J 72, 9, laet, lâl( $h) \mathrm{W} 397,28$. 412,6. H 123. J 50, 41 mit apokope des -e, lâtten $\mathrm{H} 121$, lâten $\mathrm{W} 413,29$ und p. p. lât W, H 45. 131, skêda, *sprêda); andrerseits aber weist die schreibung nête W (praet. zu nêda 'zwingen') auf langen vocal; wahrscheinlich hatte sich in der thberlieferten periode die kurzung schon entwickelt, doch wurde die consequente durchfuhrung

s. v. biriind), und dem flectierten p. p. eynle, -en, aynta J 2,30. 9, 2. 18, 10. 11. 16. 47,4 . Wegen der nämlichen erscheinungen im aofr. $\mathrm{s}$. Gramm. $\S 122 \beta$ und $\gamma$.

Ueber das verhältnis dieses thth und $t$ zu dem $g d(d d)$ und $d(d)$ in ags. cýjde (cýdde), ládde, sende etc. und as. kûthda (cûdda), lêdda, sanda etc. hoffe ich nächstens bei einer andern gelegenheit zu handeln.

1) Vgl. die stelle dà binnētlent (lies biněttent) da Fresen mitla liue ende $\hat{e} f t e r$ bifuchten hiă et mitta hânden manlike.

Statt bin $\bar{e} t t e n$ erscheint ein abnormales bynêden an der parallelstelle zu W 440,19, nümlich $\mathrm{H} 66$. Die form ist neubildung nach dem praesens, wie das p. p. beschêde (flect.) J 2, 24 für beschaette. 
derselben noch verhindert durch einwirkung des $\hat{e}$ der praesensformen mit einfachem dental.

Quae(n), quaet(b), qwê, quâtha. Statt queda inf. H 94 aus *quetha, wozu quede praes. opt. $\mathrm{J} 13,38$, finden sich in unsern quellen die formen quaen, qwaen $\mathrm{W}, \mathrm{J} 63,2$, quâ $\mathrm{J} 3$, 4. 5. 6, 2 und qwê opt. W, während als pl. praes. ind. ausschliesslich quaeth, quaed W 77, 9. 425, 33. H 1591) erscheint. Die eine oder die andere praesensform als factor fur diese ubersiedlung nach dem typus dwaen, dwaet( $h)$, dwê, gaen, gaet $(h)$, staen, stael $(h)$ geltend zu machen, wüde schwer halten; und so müssen wir behufs einer deutung erwähnter neubildungen unser augenmerk auf die praeteritalbildungen richten. Ein praet. zu queda nun ist in den denkmälern, sofern mir bekannt, zwar nicht belegt, doch darf man dasselbe fur den plur. und opt. nach sêten, -e W 440,6. 41, 2. H 65 , seet opt. J 36, 16. 18, bêden $\mathrm{W}, \mathrm{H} 64$, wêren, -e $\mathrm{W}, \mathrm{H}$ und $\mathrm{J}$ passim, treed opt. J 58 31, sprêken W, H 25. 64. 175, spreeck opt. J 46,38. 64. 59, 20, breeke, brêcke $\mathrm{W} 431,3.425,5$, breeck, brêck opt. J 58,31. 64, 12, vrêke W 79, 27 etc. (zu sitta, bidda etc.), ohne bedenken als quêden, -e ansetzen, für den sg. ind. (1. 3. pers.) als quêd vermuten nach seet $W 441,6$. H 68. J 37, 7, scheer W 398, 7, spree (c)k W 29, no. 13. 433, 10. 436, 10. 438, 28. 439, 11. 20. H 16. 24. 28. 64. 156. 175. 176. J 44,1 , breeck W 423, 22. 438, 28. H 28. 145. *Quêden, -e aber berubren sich formell mit den praeteritalbildungen dêden, $-e$ (mit $\hat{e}=\hat{a}$ des ahd. tâtum, $-\hat{\imath}$ etc., vgl. nwfr. diene plur.); *quêd sg. ind. ebenfalls mit dem sg. dêd (nwfr. die), in sofern die praeteritale form mit apokope gesprochen wurde (s. W 391, 7. 431, 20. 21. H 169. J 44, 1 und vgl. deeld $\mathrm{W} 392,8$. J 3,17, wîsdma $\mathrm{W} 439,9$, wŷsd $\mathrm{J} 27,1$, schyd (s. zu scehan), meend $\mathrm{J} 12,18$, leend $\mathrm{J} 7,2$, selle [seth] $\mathrm{W} 435,23, w d y d$ W 440,17. 26. 441, 26, brocht W 429, 7. J 15, 82, brochtma W 439, 12. 440, 18, leyd W 426, 33, leydma J 50,41, seid $\mathrm{H} 63$, sield 'verkaufte' H 154, leerdse $\mathrm{H} 65$, heed 'batte, hätte' W 398, 6. 421, 5. 433, 15. 439, 12. H 57. 117. J passim, hyed 'hătte' J 1, 1463,9 , sculd W 47, 12. H 85. 177. J 46, 38 , scolden [scold] W 424, 23, schold J 1,30. 15, 26. 44, 1.

1) Qwaed in $S 385,6$ steht an einer verderbten stelle und kann demnach nicht als beleg dienen. 
64,12, schoutd $\mathrm{J} 75,6$, $\breve{o c h t} \mathrm{~W} 420,17$, mocht $\mathrm{W} 434,10.391$, 8. J 17, 2, mucht J passim, wold W 430, 14. 18. 30. 31. J passim, woed 'wollte' J 26,17. 44, 12, woldhi H 157, schaet J 1, 46, laet, lât $(h)$ W 397, 28. 412,6. 422, 23. H 123. 144. J 5n,

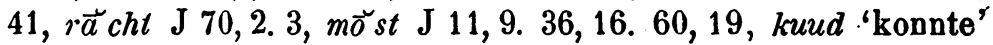
$\mathrm{J} 54,6$, wist J 15,34. 17,11. 25, 15, ênigad W 437, 19, stôwad W 397, 29, maked, -eth J 26, 12. 37, 4. 46, 38. 46. 46, 63. 64, câped, -el J 7,11. 15, 27, dulget(h) J 44, 1. 58, 34, wrsâmet J 46, 59, ordineret $\mathrm{J} 80,2$, mannet $\mathrm{J} 50,41$, wrwyeldiget $\mathrm{J} 44,1$, tylath 'zeugte' J 50,46, lawiget $J 50,19)$. Dieser umstand konnte die entwickelung eines inf. und pl. praes. ind. mit $\hat{a}$ und eines opt. praes. mit $\hat{e}$ nach dem muster von $d w a e n, \operatorname{dwaet}(h), d w \hat{e}$ veranlassen. Fưr quâ als compromissform aus quaen und queda vgl. das oben s. 392 behandelte iae 'geben' aus iaen und ieva.

Eine andere aus der vermischung der beiden formen hervorgegangene bildung begegnet in quâtha $\mathrm{H} 44$ (2 mal; das th als rest der alten orthographie), das selber wider als doppelform zu queda, zu der zeit wo das gleichbedeutende spreka ausschliesslich oder doch als norm galt (also noch nicht, wie in der uberlieferten periode, nahezu gănzlich durch ein aus der 2. 3. sg. * sprecst, sprect verdrängt war) ${ }^{1}$ ), neben den formen mit $e$ ein sprâka, -et $\mathrm{H}$ 133. 162. 164 hervorrief. (Nach dem muster von sprâka etc. entstanden dann neben *breka etc. ${ }^{1)}$ brakath, brâkanda H 81. 180).

Nach quaen, quaeth $=$ dwaen, dwaeth, gaen etc. mit der 3. sg. praes. ind. dêt, geet, steet (8. oben s. 392), ist auch das passim erscheinende quel $(h)$ als neubildung mit langem vocal anzusetzen.

Lenth in dat oen da himrick (dorfgemeinde), deer dis

1) Wegen der nabezu constanten formen sprecka, -en, brecka, -en, -e, -ed s. W 394,38 . $397,20.413,28.418,13$. 425, 19. 432, 18. S 446, 5. 455, 10. 483, 4. H 53. 55. 61. 99. 120. 133 etc. J 1, 6. 19. 22. 34. 12, 1. 13, 12 etc. (sclten spreka, z. b. S 447, 27. 457, 1. B 207. 256) und W 398, 9. 410, 11. 414, 21. 425, 5. 431, 29. 480, 16. H 58. 124. 162 etc. J 2, 37. Sch 239. Vgl. auch bisecka, -e 'läugnen' W 37, 20. 65, 19. 69, 31. 77, 1. 390, 25. 397, 24. $398,35.403,23.26 .404,5$ etc. H 35. 54. 93. 100. 102. 104.159 etc. J 15, 22. 62 (woneben biseka, -en, -e W 13,9. 405, 25. 419,21. 427,9. 21), stecken W 417, 21, wreck 1. sg. ind. W 438, 12 (neben wreke $\mathrm{H} 29$ ), wrecke inf. J 72, 8. Nwfr. sprecke, brecke, stecke, wrecke GJ und Bl 149. 
santis (der kirche) ${ }^{1)}$ en dis koningis ende des huismannes lând oen lenth ende leyt, dat hyt (der schulze) dis fârda iêris dêla moet $\mathrm{W} 391,18$. $\nabla$. R. ubersetzt das wort durch 'anlehnt, grenzt' und vergleicht as. hlinon, eine deutung, die weder in semantischer noch in formeller beziehung befriedigt (as. hlinod mlisste afr. hlenalh oder hlinath entsprechen). Das verb. ist derivatum zu einem adj. 'giland = mnl. geland 'land besitzend' (gebildet wie ags. zefeax, zemon, ahd. gibart, gifahs, giloub etc., vgl. Kluge, Nomin. stammbild. § 176) und heisst also eigentlich 'landbesitzer sein'. Es wird an unserer stelle per metonymiam, d. h. durch ubertragung rom besitzer auf die besitzung, dem land praediciert und steht demzufolge mit geänderter bedeutung $=$ 'zum landbesitz gehören'. Wegen des schwunds von giund der schreibung $-t h$ für $t$ s. oben s. 347 und unten s. 414 anm.

Li òcht und lǐcht 'gering'. Beim sendgericht, wo der vom bischof zum richter eingesetzte decken (haudprêster) das urteil spricht, treten als officielle kläger vom volke gewählte laien, die sogenannten eedswaran oder fogeden auf (8. W 401403 , spec. \$ 1 und 2). Ein solcher soll einerseits nach $\$ 2$ am schlusse keine klage einreichen (nicht wrôgia), bevor er das vergehen klaghaft gemacht hat (habbe $\breve{a} c h t e l$ ) im verein mit seinem priester und seinen nachbarn. Andrerseits aber soll nach $\S 3$ kein geistlicher eine klage erheben ohne beibilfe eines eedswara: Haetso di decken vrôghet bûla da eedsuara iefta di prêster iefta ander lĭchte lioed, so ne thoerma ... neen anderl iowa hodder to iechtvird nêr to seckw'd. Offenbar werden hier der decken und andere geistliche dem bisch of gegenüber als die niedrigen wurdenträger erwähnt, die, um als sendgerichtsbeamten auftreten zu können, der assistenz dazu gewählter laien bedürtig sind. Es ist demnach für $\overleftarrow{\imath} \breve{c}$ chte die aus dem mhd. bekannte bedeutung 'gering, niedrig' geltend zu machen (vgl. mhd. leihter man im gegensatz zu adelich). Die an der parallelstelle $\mathrm{H} 107$ Hwat soe di decken wroeght iefta oer liòchte lioede begegnende doppelform enthält einen auf altes durch brechung vor cht entwickeltes iu zurlickgehenden diphthong;

1) S. das Wb. s. 1000, doch fasse das nomen in dat sant to Boelswart als 'ufer' = mhd. mnl. mnd. sant ' $\mathrm{ufer}$ '. 
s. oben s. 384 und vgl. wegen der doppelformen (= aofr. liacht und $l \breve{\imath}$ chte levis) Aofr. gr. $\S 39$.

Li o(u)wa 'glauben'. S. oben s. 356 .

Lio(u)wa 'zurïk-, nachlassen, unberechnet lassen'. S. oben s. 357.

Liouwen 'becken'. S. s. 358.

Meit(b)ia. Das verbum begegnet ausser an den im Wb. s. $916 \mathrm{f}$. aus $\operatorname{Sch}$ (mit $\mathrm{S}$ und $\mathrm{Schw}$ ) citierten stellen ${ }^{1}$ ) in $\mathbf{J} 19$, 6. 21,11. 22, 12. 46, 10.68. 58, 3. Sch 545. Ag 112, wo es mit $t$, und J 21,6. 28,5. 29,8. 32, 24. 46, 18. Sch 545, wo es mit th geschrieben wird. Es hat die bedeutung 'machen, zu stande, zu ende bringen' und stimmt also semantisch mit makia, meckia (vgl. oben $\mathrm{zu}$ bihellet) überein, ist jedoch keineswegs mit demselben $\mathrm{zu}$ identificieren (vgl. Beitr. 17, 325), denn erstens findet sich von assibilierung einer gutturalen tenuis (oder media) vor dem -i-der schwachen verba 2 . klasse im afr. keine spur (vgl. für das awfr. bôkia, harkya, lîkia W, herckia J 67,6, byfleckkia J 74,4. 5. 6. 7, leckya J 1, 35, weckien J 1, 13, und hangia $\mathrm{W}$, hingia $\mathrm{W}, \mathrm{J} 1,43.80,2, t(h)$ ingia $\mathrm{W}$ etc.); zweitens wird der durch assibilierung entstandene laut durch $t s, s, t z$, $z, s c z, s c h z, s c h$, nie aber durch $t$ oder $t h$ dargestellt; drittens liesse sich so das $e i$ für $a$ (oder $e$ ) schwerlich erklären.

Dieses $e i$ der wurzelsilbe kann nur auf umgelauteten vocal (aus $a, u, \hat{a}, \hat{o}, \hat{u}$, ai oder $a u$ ) $+j$ zurlickgehn. Es wäre hier demnach eine etymologie zu rechtfertigen, der zufolge das verbum als denominativ gefasst würde zu einem mit altem suffix -ithu gebildeten abstractum $*_{m o ̂}(j) i t h u$ (= mnd. moi(e)le, moygele labor) $>*_{m e}(j) i t h->*_{m e i}(j) i t h->*_{m e i t h-}$ (wegen der erhaltung bez. herstellung des $-i$. und der entwickelung eines postrocalischen $i$ vor $j$ s, oben zu bêde). ${ }^{2}$ ) Also meit(h)ia $=$ 'labore efficere'.

1) In maytia Schw. 690 steht ay als schreibung für ei (vgl. oben s. 366); malye S 501,34 ist natülich schreibfehler.

2) Mit rücksicht auf das für den reflex von ahd. muoen, mhd. müejen zu erwartende fries. $e i$ ist für môya 'belästigen, hindern' $\mathbf{J} 1,11$. 28, môyen $\mathrm{J}$ 64, 28, môyt 3. sg. praes. ind. J 1, 37, môy(e)d p. p. J 1, 37. 28, 21 entlehnung aus sächsischem sprachgebiete anzunehmen. Das urspr. $o i-j$ dieser formen (=mnd. $\delta i-j$ aus $\hat{o}-j$ ) war nwfr. moeye (d. h. muे $i-j e$ ) zufolge nach dem muster von gróya etc. aus *gríi-ja (s. oben s. v.) in $\hat{o} i-j$ übergegangen. 
Wegen des $t$ und th unsres verbums beachte man die abstracta meent(e) 'gemeinde' W, S, H 104. J 25, 15. 16. 28, 16. 33, 5. 64, 16. Sch 334. 375. 545, brout(e) 'bräu' Sch 603. 671, daedbante 'mordklage' W (vgl. aofr. benethe), wente 'gewohnheit' J 28,14, beieerte H 29, tiaefte, tiêfte 'diebstal' W 69, 5. 421, 21. 431, 30. 428, 17. 438, 22. H 29. 93, tiuchte 'diebstal' H 74. 135. 141. 165. 169, fayte 'fehde' $\mathrm{W}$, liaefle Sch 735. 773. J 65, 1. 87,9 und meenthe $\mathrm{S}$, Sch 545, bryouth Sch 540, bannethe $\mathrm{H} 89$, bantha J 13,38, lamthe W 413,9. 13. 468, 22. 30. J 81, 22, lamethe $\mathrm{H} 139$, deenthe 'qualität' $\mathrm{J} 65,4$, dioerthe $\mathrm{H} 24$, deren th keineswegs die spirans darstellen kann, sondern als rest der alten orthographie und als zeichen für die tenuis gelten muss, weil in der therlieferten periode dem durchgehenden $t$ (aus stimmlosem $t h$ ) und $d$ (aus stammhaftem th) zufolge die spirantische qualität des alten sogenannten interdentalen spiranten in die nicht spirantische tbergangen war.1) Woher aber dies

1) Daher auch $-l(h) e$ in den nach dem muster dieser abstracta umgebildeten formen berte 'geburt' $H$ 26. 30. 161, berthe $\mathrm{H} 47.48$. W, S, J 46, 35 (aus berde H 95. 106. $117=$ aofr. berd(e), ahd. burt) und bernte 'last' W, Sch 463, bernthe J 64,13 (mit metathesis für berdene $\mathrm{B} 162=$ ags. byrgen), berthe 'last' J 32,13 (ahd. burdi).

Fuir ein solches $t h$ mit lantwert $t$ s. auch u. a.: neben normalen ting, tingia, tielda 'dulden', tank, tiaef, tremen 'drittel', taulic W 434, 29. 435, 10. 20. H 20,21 , torp, terp, tyênst, tyênia, tiùster etc. thing(h) W 392, 13. 394, 28. 425, 15. 428, 25. 28. H mitunter. J 13, 16. 81, 8, thingia, -ad H 64. 70. 82, thielda, -en W, H 2. 3, thank H 120, thiaef H 131, thremen H 41, 48. 131. 134, thaulic $B$ H, thorp und therp $B$ B3; neben toer debet $\mathrm{H}$ passim thoer $\mathrm{W}$; neben den normalen flexionsendungen -at, -et, die ab und zu begegnenden -ath, -eth; vgl. noch oben s. 408 anm. 1). Hieraus begreift sich die nicht ganz selten auftretende schreibung th für urspr. $t, \mathrm{z}$. b. in planthia 'pflanzen' $\mathrm{J} 64,12$, munthe, monthe $\mathrm{W} 3,19.5,2.37$, 30. 407, 3. 431, 9. 30. 427, 26. J 64, 14, munthere W 37, 28. 428, 29. 31, munthia W 428, 12, renthen 'zinsen' J 32, 22. 24. 33, 5. 36, 14. 37, 4. 7. 77, 3. 4, natha 'genosse' W 416, 18. 425, 15. 440, 19. 27. J 42, 4. 50,46, kirthe 'kürze' W 429, 35, betha 'büssen' W 404, 22. 23. 412, 11. 413, 28. 426, 4. 7, beth 'besser' W 397, 14. 435, 1. J 13, 26. 14, 2. 15, 78. 17, 17 etc., letha 'lassen' W 411, 26. 438,14, gretha W 411, 27, bysoechthe 'nachsuchten' W 430,5 , tuchte [tuchthe] 'däuchte' W 430,1, noth 'frucht' W $390,13.475$, 25. $J$ 64, 19. H 300. 304 , neth 'ntitze' $J$ 18, 9, bythe 'biss' $W 389,29$, wytha 'wisse' J 15, 36. 55, 1, ethber 'essbar' H 44, thioegh 'zeugniss' W 413,5 , thioeged $\mathrm{W} 412,31$, thilet 'zengt' $\mathrm{W} 405,10$, tho(e) 'zu' W und $\mathrm{J}$ mitunter. H 300, thi ' $z \mathrm{n}$ ' W mitunter, scheth 'tier' J 64, 8, opthie 'aufgehe' 
-te, statt dessen mit ausnahme von syu(e)(c)kte, sioecte, syôkte (s. oben s. 385. 387), wo der dental mit stimmlosem consonanten zusammenstiess, bei regelrechter genesis - de zu erwarten wäre? In den bildungen mit kurzer antepaenultima musste sich zur zeit der $u$-apokope im nom. sg. eine form ohne $\cdot u$ entwickeln, gegenuber den bildungen mit langer antepaenultima, wo das $-u$ haften blieb (vgl. Beitr. 17, $288 \mathrm{ff}$.). Das auslautende th der ersteren formen erlitt als solches in der periode, wo die stimmlosen intervocalischen spiranten stimmhaft wurden, keine änderung und konnte von hier aus die erhaltung bez. herstellung der qualität in den mit flexionssuffix versebenen casus veranlassen; solche bildungen aber ermöglichten das teilweise eindringen ihrer endung in die derivata mit langer antepaenultima, deren suffix regelrecht entwickelte stimmhafte spirans enthielt; (nur ausnahmsweise blieb das stimmhafte $t h>d$ erhalten, nämlich in den oben 8.346 und 356 besprochenen bêde, *griôde). $\mathrm{Vgl}$. auch mnd. (Schiller-Lübben) behôfle, dûrte, gewonte, lembte, dêfle und duvete, dîfte, rûmte, schamte, begerte, hôchte (woneben veide, hôgede), sowie dêpte, sukte, sûkte, starcte, wo das $t$ sowie so hätte entstehen mlissen; mnl. (s. meine Mnl. spraakk. $\S 110)$ begeerle, lengte, hoechte, leemle, veete, schaemte, behoefle, ghewoente, dierte, diefte, scoente etc. (woneben begerde, lengte, hoegde, leemde, veede, scaemde), sowie dicte, diepte, stercte, joecte, siecte. ${ }^{1}$ )

Mit rlicksicht auf nwfr. $t s j$ aus $t j$ in achtsje, haetsje (awfr. achtia, hatia), jachtsje, fellsje 'fassen' (vgl. oben 8. v. fatiender), tsjeaf 'dieb', tsjien 'zehn', tsjin 'gegen', tsjüuster 'dunkel' etc., könnte man beim ersten anblick vielleicht versucht sein, mit altem meitia das nwfr. meitsje 'machen' zu identificieren. Doch

H 180, wth(-) 'aus' H 300. J 1,46. 57, 8. 17. 63, 7. 64, 8, wtha, wther 'aus dem, der' J 70, 4. 58, 14, butha 'ausser' J 30,19, haeth 'was' H 89. J 2, 37. 15, 36. 72, 1. 81, 22, naeth 'nicht' $H$ 300. 301. 302. 303. 305. J 28,18, die ziemlich häufigen participia auf -ath, -eth in $H$ und $J$ etc.

1) Die a. a. o. für das mnl. -te vorgeschlagene deutung, phonetische entwickelung in diepte, dicte etc., analogische genesis nach dem muster letzterer formen in begerte etc., ist mit rücksicht auf siu(e)kte als die einzige belegte form mit stimmlosem consonanten vor dem suffix für das fries. nicht anwendbar. Was aber fiir das fries. gilt ist selbstredend auch für das niedersächs. und niederfränk. geltend zu machen. 
müssen hier bei näherer betrachtung zweierlei umstände stutzig machen: zunächst dass zu meitsje die formen mekke und makke, mekkest, -et und makkest, -et als praet. und p. p. (aus *mecked, *macked fur meckade, -ad, mackade, -ad, s. oben zu byhellet), bez. 2. 3. sg. praes. ind. stehen ( $G J$ und $B l$ 155); sodann dass das ziemlich junge $t s j$ aus $t j$ in denkmälern, die schon meitsje aufweisen, nicht begegnet: G. Japicx schreibt neben meytsje immer achtje, haetje, jachtje, fettje, slachtje, treastje, tjeaf, tjien, tjuester etc. und in Wassenberghs im anfang dieses jahrhunderts in gemeinwestfries. dialekt verfasster ubersetzung von Matth. 6 finden sich neben meitsje (s. Wassenberghs Taalkundige bijdragen 1,143) trompetje, festje 'fasten', tjueuster, haatje, tjean, tjinje, forachtje. Ausserdem aber liegt eine anderweitige erklärung unseres meitsje auf der hand bei beachtung des nwfr. lautgesetzes, dem zufolge altes postvocalisches $c k j$ zu itsj wurde; vgl. nach GJ und $\mathrm{Bl} 155 \mathrm{f}$. smeitsje, weitsje, reitsje 'treffen, hinkommen' (awfr. smeckia, weckia, s. oben zu byhellet, und durch die nämliche genesis entstandenes * reckia aus *rakia $=$ mud. mnl. raken 'treffen' aus *rakôn) mit praet. und p. p. smekke (später auch smakke nach makke zu meitsje), wekke, rekke (aus * smecked etc. fur altes *smeckade, -ad etc.) und 2. 3. sg. praes. ind. smekkest, -et (später auch smakkest, -et), wekkest, -et, rekkest, -et; láitsje (laeylsje) 'lachen' (awfr. hlackia, s. oben s. v.) mit praet. und p. p. lake, 2. 3. sg. praes. lakest, -et ${ }^{1}$ ); ploaitsje (d. b. plóaitsje) (awfr. ${ }^{*}$ plockia = ags. pluccian) mit plôke praet. und p. p. ${ }^{2}$ ), plokst, plokt 2. 3. praes.; [vgl. auch solches itsj aus $k j$ in koaitsje, loaitsje (awfr. *kokia, *lôkia = ahd. chohhôn, ags. lócian) mit kôke, lôke und kôkest, -et, lôkest,

1) Merkwturdig ist hier das einfache $k$ ( $\nabla g l$ auch die doppelform laeckje, d. h. lae-ckje mit ck als schreibung für $k$ ), das sich nur durch einwirkung der doppelformen makade, -ad und mackade, -ad (s. oben s. 349) erklärt, welche neben (h)lackade, -ad, (h)lakade, -ad hervorriefen. In 'Vrymoedygheit van en huisman' (s. s. $417 \mathrm{anm}$. 2) steht (s. Wassenberghs oben citierte Bijdr. 1, 157. 164) (be)lakje, das nach der in diesem denkmal üblichen orthographie als (be)lakkje zu lesen ist.

2) Einfaches $k$ nach den gleich oben zu verzeichnenden kôke, lôke zu koaitsje, loaitsje. $\mathrm{Zu}$ ploaitsje kam in der jetzigen sprache neben plôke als praet. auch ploaite in schwang nach analogie von praet. achte, haete, fette etc. zu achtsje, haetsje, fettsje etc. 
-et]. 1) Also meitsje aus meckia (s. oben zu byhellet). ${ }^{2}$ ) Auffallend ist hier indessen erstens das fehlen eines nach makke, -est, -et zu erwartenden, auf mackia zurückgehenden *máitsje (mit ái, wie in láitsje), zweitens die bevorzugung des im jungeren nwfi. allein- oder wenigstens nahezu alleinherschenden makke, -est, -et gegenuber den e-formen mekke etc., die, als zu meitsje gehörend, grade in wekke, rekke etc. zu weitsje, reitsje eine stütze bätte finden müssen. Für die erstere erscheinung ist wol anlehnung des verbums = 'machen' an meye 'können' in anschlag zu bringen; für die andere möchte ich, auf den im nwfr. auf schritt und tritt zu beobachtenden einfluss des nl. hinweisend, einwirkung von seiten des $a$ im ndl. maken ins auge fassen.

Moetlick 'recht, billig'. S. zu oenmoetlik.

Nâth, naethilyka 'ebenbürtiger'. J 50, 46 enthält folgendes juridisches rätsel nebst lösung: Deer wêren trê brôren ende wêren alle folbrôren ende nas neen anderis natha (gen. pl.) (und keiner gehörte zu den genossen des andern, war des andern ebenbürtiger) ende deelden ênen (lies ênes) faders gued ende môders. Dat (lies Dat was) deeromme schepen [scepen]

1) Neben laeytsje und loaitsje stehen bei G. Japicx auch laeckje und loackje mit erhaltung der alten form durch anlehnung an das praet. und p. p. laecke, loacke (ck als schreibung für k). In lockje, schrickje, stickje, flekkje (awfr. fleckkia, s. oben), rekkje, leckje ist das kk uberhaupt erhalten geblieben.

2) In der Molkwerumschen ïbersetzung des cap. 6 des evangeliums Matthaei (ende des 18. jh.'s, s. Wassenberghs Bijdr. 1, $140 \mathrm{ff}$.) steht meatje (s. 142, v. 16), dessen $t j$ als zeichen für $t s j$ $z u$ fassen ist nach sietje 'suchen, -et' (s. 146, v. 32. 33), d. h. sietsje (aus "sêkja[n]) mit assibiliertem guttural.

Der nwfr. (Zuidhoeksche) dialekt, in welchem der (ziemlich alte, jedoch nicht genau zu datierende) aufsatz 'Vrymoedygheit van en huisman' (s. Wassenberghs Bijdr.1,151-169) verfasst ist, hat maaikje (s. 152. 153. 156. 157. 160) < awfr. makia (s. oben s. 348) und scheint tiberhaupt die in rede stehende jüngere assibilierung nicht gekannt zu haben; vgl. daselbst raaikje (s. 160) ans awfr. *rakia, sowie (be)lakje (s. 157. 164), kookjende (8. 163), wo die in den zwei ersteren formen entwickelte $i$-epenthesis unterblieb in folge von anlehnung an die praeteritalbildungen *lakke $(d)$, *kooke $(d)$.

Ebenso hat der jetzige dialekt von Hindeloopen moecekje (Winkler, Nederduitsch-Friesch dialecticon 1, 446). 
(das verhielt sich so): datter een man was, ende (der) hêde een aefft wŷff ende taegh (zeugte) bij een ôdera wîff een bern; $d \hat{a}$ sterff sŷn aefft wŷff, ande taegh (er zeugte) thaueswisa (seiner gewohnheit, d. b. seinem gewohnten umgang mit jener frau gemäss) eèn oer bern; deerney noem hij hiŏ (sie) to aefla wîve

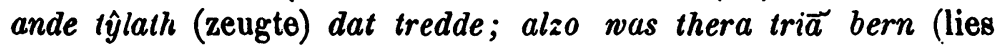
berna) neen ôderis naethlyka [naethilyka].

Wegen nâl(h) fưr *ginât (ags. ¿̇enéal, as. genôt, ahd. ginôz) 8. oben zu bêde; wegen der schreibung th für $t$ in natha und naethilyka s. oben s. 414 anm.; wegen des -a von -lyck beachte gâstelick J 1, 21. 31. 34. 15, 1, wylelick J 1, 9. 5. 2, 5, tnîvelick J 1, 50. 13, 15. 36. 39. 22, 19, (on)rîchtelick J 1, 5. 2, 7. 15, 71. 46,33, nôgelick J 2,37. 12,31. 34. 13,8. 15, 23. 38. 40. 55. 77. 21, 8, byschêdelick J 13, 7, redelick J 28, 4. 29, 2. 3. 46, 52. 64, 18. 67, 2 etc. neben gastlick J 2, 14. 13,1. 15, 3. 16. 19, witlyck J 13, 16, twŷflick J 13,2, rîchtlick J 29, 3, noeglick J 13, 10. 41. 14, 4. 17, 8. 46, 1. 18. 22, byscheedlyck J 46, 75, reedlick J 28, 1. 14. 17. 46, 49. 60 etc.; wegen des -i- als schreibung fur -2- in J ondir 'unter' 2, 28, dêdir, rêdir 'täter, beräter' 62,3, sprekir passim, câpit, prowit, beswêrit, onteerwit p. p. 11, 3. 32, 5. 9,6. 19,3. 46, 33, fregit, câpit, byjarith 3. sg. praes. 11,4. 7 . 15, 26. 32, 12. 1,11 etc.

Zur beleuchtung obiger lösung sei auf $\mathrm{J}$ 47, 5 hingewiesen: de natuerlike sonen moghen aefft w'da twyrahanda [twirahânda] $w \hat{y} s$...; int aerst so aeffliget (beiratet) hij da frowa, deer hij da natuerlike kŷnden bij crijn [teijn] (gezeugt) haet, so sint da $k \hat{y}$ den aeffl, deer hij eer teyn haet ende eefter tîcht bij der frowe.

Nenterâ, ninterâ 'keiner von beiden'. S. s. v. enterâ. Nyoed, nyoedsecken 'not, zwang'. S. s. 386 anm.

Nômad. S. \&. v. binaemd.

Oenmoetlick. v. R. ubersetzt das adjectiv (Wb. 1105) durch 'angemutlich' und vergleicht ags. unmód discors. Die richtige bedeutung von moetlyck geht hervor aus $\mathrm{J} 13,12$ Hweerso dij riachter da twŷn iggen (parteien) tofara hine hevet myt riâchta layenghe [laynghe] bröcht and sy $\vec{a}$ ayderne op ôrem sprekket, dat hij ayderen allŷck eenstich (gewogen) schel wessa and lyck riachtes, and hat so (was) da clager moetlick is, dat 
schel wessa moellick da sitter (beklagten) bêda in beschermense ende onder wederclaegh. Also 'was dem kläger recht ist, das soll auch dem beklagten billig sein'. Vgl. ahd. muoza fas, licentia, facultas.

Dieselbe bedeutung bez. die negation derselben trifft auch $\mathrm{zu}$ an den beiden folgenden stellen: het (was) naet ôrberlyck is off nette da lânde nêr naet moetlyck da lyuedem, dat thoer ma to neen riûcht hâlde $\mathrm{J} 75,9$; Haet (was) is der êrwa riòcht? Eerlic thing rêda ende creflelic [treflelic] (s. uiber dies adjectiv unten s. v.) thyng biada, wrbiêda dat onriacht, henzia (genehmigen) moetlikera thingha (gen. pl.) ende aeck by hwîlum oenmoetlikera thingha om anxta des ierra (des schlimmeren) H 21 (s. noch die parallelstelle $W 435,18) .{ }^{1}$ ) Ebenso in der aofr. F 4 Hwet is there êwa riûcht? Êrlike thinga rêda, craftelic [trâstelic] thinga biâda, urbiâda unriûcht, henzia mêthlika (lies môthlika) thinga and ac bi hnôlum unmôthelika ${ }^{2}$ ) thinga thruch fruchta thet (lies thes) wirre.

On(bi)naemd. S. zu binaemd.

Onbescât. S. s. v. wrichte 'arbeiter'.

Raefferde. S. zu freed fussn.

Rekenad. S. za hensich.

Rêsien und rêset in opdat ma neen quaed nêr eergh op

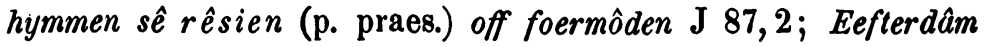
dat een man een gued off lând câpet fan dajenen, deer (den) hij den riâchta hêra rêset (Hettema las hier naet fan is) ende meent oers naet, so hijt fan da rî́chta hêra câpet haet J 36, 4. Beide formen gehören zu einem inf. rêsia 'vermuten', das mit synkope des $w$ vor $i$ (wie in stoya, scoya, s. Beitr. 17, 566) zurluckgeht auf * rêswia $(n)=$ ags. nach ráswodon commenti sunt (Beitr. 9, 292) zu folgernden *róswian.

Nach awfr. rêsia muss die flexion von ags. ráswodon als eine urspringliche, nicht als eine nach art von smirode, besyrode, fremode, dwelode etc. (Beitr. 9, 288. 292) entstandene

1) Wo êrlykera und treftlykera nach $\mathrm{H} 21$ in êrlyka und trefllyka (acc. pl. ntr.) zu corrigieren sind.

2) Wegen des aofr. für $t$ stehenden $t h$ (das auch in $R^{1}, B^{1}, B^{2}$, d. $h$. in quellen erscheint, die kein $t$ für altes th aufweisen, mithin eine andre erklärung erfordert als das awfr. $t$ ftir $t h)$ vgl. Aofr. gr. $§ 119$ anm. und $\$ 124$. 
gelten, denn ein ubertritt schwacher verba 1. klasse in die 2. klasse ist fur das afries. nicht nachzuweisen (ausserdem liesse sich eine solche ubersiedlung des verbums auch im ags. kaum begreifen, weil eben der die entwickelung praeteritaler formen mit -o- $(-a-)$ vermittelnde factor, ein praes. auf -ian, hier nicht denkbar wäre).

Sa(u)wen, saun, sân. Aus der oben s. 359 sowie zu fliueswerp und unten zu tro(u)waden erörterten lautentwickelung ou durch $o$ und $\hat{o}$ aus $a$ und $\hat{a}$ vor ursprunglichem oder aus $v$ entstandenem $w+\vec{o}(-)$ oder $-u(-)$ und $a u$ aus $a$ und $\hat{a}$ vor $w+$ $a$ oder hellem vocal, erfolgt der schluss, dass in den flexionsformen der einschlägigen verba und nomina in einer bestimmten vorgeschichtlichen periode ou mit au wechselte. Ausnahmsweise haben sich diese doppelformen bis in die historische periode erhalten, in der regel jedoch erscheint hier in folge von ausgleichung in ein und demselben verbum oder nomen entweder ou oder au: scounia etc. und scaviane, houneth etc. und hauwen etc., tow und tau $(w)(e)$, towerie und thauwerje (oben s. 377 und 359), stouwia und stawia (= staunia? s. zu trouwaden), doch trouvaden, *kouwia (s. zu trouwaden) und taewiane, bidauen, clau, claw (a), tawa, -em, blaun, thau, awane, thawa 'zeigen' (s. oben s. 377 ff.), hauk, naula, *lauwigia 'laben', kauwelje, haud, lauwa 'nachlass', lauwigia 'nachlassen', (by)rauwia, lauwa 'glaube' (oben s. $353 \mathrm{f}$. und 359). Dass aber zur zeit der gedachten doppelherschaft neben den bildungen, denen ou aus $e$ vor -wo, -wun aus -vo, -vun etc. oder ou aus $\vec{o}$ vor $w$ aus $v$, doch kein au zukam (oben s. 360 und 351), nach dem muster der ou- und au-bildungen eine form mit au aufkommen konnte, liegt auf der hand. Und in der tat finden sich zwei in der weise entwickelte formen: das in unsern denkmälern passim begegnende sa(u)wen, saun (mit sauwenda, saunda, sauwentich, sauntich, saunfald etc.), welches sich nicht nur existenzberechtigung erwarb, sondern sogar dermassen vordrang, dass das alte sowen im awfr. ganz selten wurde, im nwfr. gänzlich ausser gebrauch kam; und nwfr. lauwje 'loben', belauwje 'versprechen, geloben' GJ = awfr. *(bi)lauwia neben louwed (oben s. 352) und lowiad etc. (oben s. 363), insofern hier on = oun vorliegt.

Neben saun, sauntich etc. erscheint in den alten quellen 
einige male sân- (in sânderhânda J 13,2, (t)sântich Ag 63. Sch 549. 646. 647. 657. 658. 659. 660.664. 680, sânthiêne Sch 395), das selbstverständlich nicht als rein phonetisches erzeugnis zu erklären ist, sondern als analogiebildung nach altem *tiân 'zehn', *tiàda (wofür in der luberlieferten periode tiên, tiênda W 7, 29. 17, 5. 20. 25. 45, 5. 49, 17. 59, 17. 410, 19. 430,17. 18. 432, 8. 438, 13. 24. 31. 462, 15. J passim. Sch 657 etc. und tiaen, tiaenda W 438,5.6. S 483, 7. H 71. 75. 84. 85. 91.151. 168 etc. mit doppelschreibung zur darstellung des als $i \hat{e}^{a}$ gesprochenen früheren $\left.i \hat{a}_{.}{ }^{1}\right)$ Im nwfi. steht sân, sânde etc. neben saun, saunde ete. GJ.

Ein seitenstiuck zu den erwähnten analogiebildungen mit

1) S. noch biêda, -en, -e etc. W, S, H und J passim und biâda, -e etc. S, W 400, 23. H passim, $w r$-, fo(e)rliĉsa, -en etc. W 429,21. J 8,5. 25, 12. 18 und wrliâse, -ath H 166. 173, tziêsa, -ane etc. W, H 99. 115. 152. J 21, 1.6. 26, 20 und tziàsane, -ath H 159. 173, niêla, -en W, H 160. J 15, 71. 51, 3. 58, 34. 62, 6 und niata $\mathrm{H}$ 108. 145. 174, biêr W, S und biâr H 142. 242, diêr W, J 64, 8. 18 und diaer H 84. 90. 243. J 64, 16. 17. 18, dîêp, diêpe $\mathrm{W}$ und diaepe $\mathrm{H}$ 85. 173, syêck, sieek W, H 98. 106. 115. J 72, 9 und siaeck $\mathrm{H} 83$, stiêpfader, -môder W, J 50,41 und sliaepfadir, -môdir H 117, tiêf 'dieb' mit tiêfte W, J passim und $t(h) i a c f$, tiaefle W

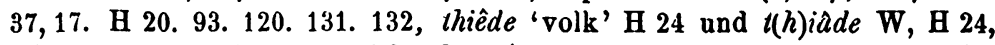
tiêder 'bindseil' W und tyâder S, tyêl 'rad' J 59, 18 und tial H 148, tiêgmerg, -schonck W, J 59,18 und thiâchmerch, -scunke S, H 237, tiênia, -ade etc. W, H 66. J 45,13. 86, 6, tiènst W, J 1, 40. 44, 10. 45, 8. 56, 1. 64, 25. 81, 11, tyêner J 37, 9 und tiânia S, H 29. 105. J 46,48, tiaenst H 51 . 64. 105. 106. 115. 303, niêr 'näher' (s. oben s. 403). W, S, J 50, 20. 21. 24. 53, 1. 54, 3.4 und niaer $\mathrm{W}, \mathrm{H}$ und $\mathrm{J}$ passim. Constante auf $i \hat{a}$-qualitït hinweisende schreibung begegnet aber: in liaef, -lick, -te $\mathrm{W}, \mathrm{H}$ und $\mathrm{J}$ passim (wol durch einwirkung von $i \dot{a}$ des triphthongen in lyauve etc., s. oben s. 363); in liaecht, liach W, S, H 28. 103. 118. 120. J 13,46. 59, 1. 65,8, fiaerda 'vierte' W, S, H 67. 71. 83. 85. 116. J 12, 5. 50, 42. 56, 1 . 80,15 (offenbar durch conservierenden einfluss des cht und $r \cdot d$ ); in den verbalen flexionsformen, worin der zweite component gleichsam die endung vertritt, nämlich siae(u), siaene, siâne, siaende 'sehen' überall passim, tiaen, tiane, tiaende 'ziehen' uiberall passim und tiaed, tiael(h) plur. W, H 159. 164. J 50,41, iân, iaen 'bekennen' (8. zu scehan fusso.), iaen etc. 'geben' (s. zu geva), tyaende 'in ordnung' (s. zu hensich) (durch einwịkung von gaen, staen, gaeth, staed, s. oben s. 392); in hliapa (s. s. v., durch anlehnung an die verba 6. klasse); und in den formen mit auslautendem diphthong fia 'vieh' etc. J 42, 7. 59,1. W, H 78. 146. Sch 250, hi $\bar{\imath}$ acc. sg. fem. und nom. acc. pl. (hiernach auch in fias W, S, H 242. Sch 250, hiàra, -e gen. pl., hiârem dat. pl.). (Wegen fîand s. oben 
au für ou bildet das indef. iou(we)lick, jowelik quisque $\mathrm{H} 243$. W 472, 30. S 447, 11. 456,17. Sch 517 neben ia(u)welik H 242. $\mathrm{S} 447.456,7$. Nur letzteres ist das resultat rein phonetischer entwickelung als die form, welche durch die wirkung des oben zu fliueswerp erörterten gesetzes hervorgehen musste aus *iâwelik = aofr. iâhwelik (aus *gi-â-hwelik, also zum teil ahd. iohwelîh, zum teil ag8. zehwilc entsprechend, s. Aofr. gr. § 254). [Wegen des neben iou-, iaunelik verwanten iên(e)lick, iêv(e)lick J 2, 11. 25, 28. 45, 6. 72, 9 (Hett. falsch jowlick für jênlick). W 471, 17. $472,32=$ aofr. iêwelyc aus *gi-ê (immer) -hwelik vgl. das oben s. 379 uber die nichtafficierung des $\hat{e}$ vor $w+$ hellem vocal bemerkte.]

Seerfâldich. Der zweite teil der composita mhd. sorgvellich, mnd. sorchvaldich, -veldich, -voldich, angestvoldich, monfrk. sorgh-, anxtfeldich (Teuthonista), mnl. sorch-, anxtvoudich ist hinsichtlich seiner bedeutung von dem mit zahlwörtern verbundenen -veltich, -valdich etc. zu trennen und als derivatum zu valte, valde, volde, voude 'runzel' zu fassen; also die composita $=$ 'durch sorge, angst erzeugte runzeln habend'. Ein hiermit ubereinstimmendes awfr. sorchfaldich kann ich aus den mir zur verftigung stehenden quellen nicht belegen; dass dasselbe jedoch in der älteren sprache in schwang war, ist zu folgern aus dem bei G. Japicx begegnenden sorgfadich (vgl. im nämlichen denkmal yenfâde, sânfâde, tuwsendfade und beachte wegen der l-synkope daselbst kâd 'kalt', wrâd 'welt', hâde 'halten', $\hat{a d}$ 'alt' etc.). Nach dem muster dieses *sorchfaldich aber entstand ein mit seer dolor gebildetes seerfâldich, das = 'traurig' begegnet in seerfaldicheed: Een frow (d. b. eine witwe), deer bynna der $t \hat{y} t$ dis weermôdis ${ }^{1}$ ) off der seerfaldicheed, dat is bynna da scrŷljeer, is haesten ende nympt een ôren man, $d y \widetilde{o}$ is . . e eerloes $\mathrm{J} \mathrm{87,1.}$

B. v.). Dass auch hier jedoch in der folge afficierung eintrat, lehren die nwfr. in GJ verzeichneten ljeaf, ljeacht, sjean, tjean, jean (neben jaen), ljeappe, jae (neben ja), d. h. ljêåf, ljêa acht etc. und jêa. (Die durch fortgesetzte, nicht gleichmässige qualitätsänderung aus biêa $d a$, biêrar etc. hervorgegangenen nwfr. formen und die in der jetzigen sprache für sjean etc. eingetretenen erfordern eine besondere erörterung, die ich mir für eine spätere gelegenheit vorbehalte.)

1) Vgl. mhd. widermuot, mnd. weddermôt 'trübsal'. 
Aus derselben von *sorgfaldich ausgehenden beeinflussung wird auch die bedeutung 'sorgfältig' begreiflich, welche seerfâldich beizumessen ist in Alle riûchteren âgen to sêken da wird ende da to folgien, also fŷr so hy $\vec{a}$ mogen, ende dat schelletse dwaen myt seerfâldigen [-er] herte $\mathrm{J} 2,13$.

Sy $\hat{u}$, siow. Für dieses von Buitenrust Hettema, Taal- en letteren 1, 249 und von Bremer, Beitr. 17, 335 besprochene wort = 'signal' hat letzterer richtig etymologischen zusammenhang mit siân videre erkannt. Wie aber erklärt sich diese zusammengehörigkeit? Das nomen ist offenbar ein substantivisch verwantes verbaladjectiv, welches auf altes *sêni, flect. *sênjzurlickgeht (wegen ursprtinglicher oxytonierung solcher deverbativa vgl. an. frógr, ags. $y$ y/ynde). Altes * sêni musste dem für das $w$ vor $i$ geltenden synkopierungsgesetz gemäss *sê ergeben, aus den flectierten formen aber musste *sêrv- hervorgehen, woraus ein neu gebildeter nom. acc. sg. * *êt $n$, der nach dem oben s. $378 \mathrm{f}$. erörterten gesetz zu *siu wurde und als solcher die neubildung eines flectierten *siune, -es veranlassen konnte, woraus in der folge *sioune, -es und siou (sion).

Siûth, siôth, syoet, siêth 'schiesst'. Die Beitr. 14, 269 vorgeschlagene annahme eines zur 2. starken klasse gehörenden afr. *siâwa = 'stechen' ist zu verwerfen: vor $w$ stand nur $i \hat{u}$ (vgl. Aofr. gr. \& 24 und beachte fur das awfr. $i(\hat{u})$ we poss. sowie $\operatorname{tro}(u) w e$, rio $(u)$ wen etc. aus *iriûwe, *hriûwane etc., oben $\mathrm{s.}$ 351 anm.); die neben der 3. sg. praes. ind. siuth (syoet, siôth) begegnende doppelform siêth S 443, 3 wäre also mit *siâna unvereinbar. Alle drei formen begreifen sich indessen bei der annahme eines alten *sewan: die hierzu gehörende 3. sg. praes. ind. *servith wurde durch die $w$-synkope zu *sêth und hieraus entwickelte sich eine neubildung *sề $a(n)>*_{\text {siae }}(n)$ ( (vgl. *miae $(n)$, *lyae(n), oben s. 402), das nach dem muster von gaen, staen mit $g \hat{e} t(h)$, stêt( $h)$ die genesis eines siêth für *sêth hervorrief; neben der neubildung *siae $(n)$ aber konnte sich das alte *sewa $(n)$ balten, woraus in der folge durch das oben $8.378 \mathrm{f}$. besprochene gesetz * siana mit hierzu gebildeter 3. sg. praes. siâlh $\mathrm{W}$ und durch analogische entwickelung (s. oben s. 386) siôth H 227. $\mathrm{S} 456,18$, syoet $\mathrm{S} 348,21$. Siath 3. sg. S 447, 10 ist eine unmögliche form und muss als schreibfehler für siêth oder siôth 
gelten. 1) Anstatt der Beitr. 14, 269 angesetzten bedeutung 'stechen' ist unser verbum richtiger als = 'schiessen' zu fassen, und zwar mit rlicksicht auf die verwanten aid. sâumi 'ich treibe

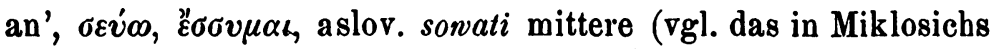
Wb. citierte sulicami sowachomu $\left.\beta \varepsilon^{\prime} \lambda \eta \dot{\alpha} \varphi \dot{\eta} x \alpha \mu \varepsilon v\right)$ : * sewan $\mathrm{zu}$ aind. sâumi, wie got. sniwan zum athematisch flectierten praehistorischem *snômi (s. Streitberg, Zur germanischen sprachgeschichte s. 101.2)

$\mathrm{Zu}$ *sewan gehört als deverbativ aofr. siâma 'schusswunde' mit siâ aus *sia- aus *sio- aus *seo aus *seu aus *sen-; vgl. brecma, frelma (Aofr. gr. § 184 s. 150). Die daneben begegnende seltene doppelform sima ist die folge von anlehnung an die dissyllabische vorstufe zu *siân, nämlich *si-a(n).

Das Beitr. 14, 270 in ûtesên geänderte âtesîn Huns. 86, 5 ist als die richtige lesart aufrecht $\mathrm{zu}$ halten. Die bedeutung des p. p. ist nicht 'ausgestochen', sondern 'ausgefallen'; vgl. die parallelstellen in $\mathrm{R}^{1}$ Jef hit (das auge) is al atekwnken (geschwunden, s. Gramm. $\S 270 \alpha$ ) und $\mathrm{E}^{1}$ Gef hit is al ute. Gunther (Die verba im altostfries. s. 23) war also im recht, als er die form auf *gisigin zu *sìga (= awfr. sîga 'fallen, sinken') zurückfubrte. Bremers vermutung, es sei âtesnîn (aus *-snithin) $\mathrm{zu}$ lesen (Beitr. 17, 334), ist unhaltbar nicht nur wegen der erforderten bedeutung 'ausgefallen', sondern auch weil im überlieferten Hunsigoer dialekt die synkope von intervocalischem th oder $d$ eine völlig unbekannte erscheinung ist.

Scehan (die flexion des alten). Als die praesensformen dieses verbums durch die wirkung des gesetzes ' $i$ zu $e$ vor dunklem vocal der folgesilbe' ${ }^{3}$ ) zweierlei wurzelvocal erhalten hatten, konnte entweder das so entwickelte verhältnis gehandhabt werden oder der eine oder der andere der beiden vocale durch

1) Einen rest des alten *siae(n) bewahrt das in GJ verzeichnete (und auch in der jetzigen sprache noch geltende) sjette 'schiessen' (mit tt wie awfr. scietta, -en Sch 603) $=2 \mathrm{wfr}$. ${ }^{*}$ sietta, mischbildung ans ${ }^{*} \operatorname{siae}(n)$ und scietta $<{ }^{*}$ skiala.

$\left.{ }^{2}\right)$ Die richtigkeit des a. a. o. über got. $v$ für $g g w$ bemerkten möchto indessen $\mathrm{zweifelhaft}$ sein.

8) Vgl. iân, iaen 'bekennen' H 42. 59 (= ahd. jehan), tiaen und tiên decem, siaen 'sehen', fià 'vieh' (s. zu sauwen) und s. für das aofr. Gramm. $\S 6 \delta$. 
ausgleichung sein gebiet erweitern. Conservativer neigung fröhnte in diesem fall das ofr. schida(n) inf., skiâth pl. ind. und skêt(h) sg. ind. (aus *skehith mit $e$ aus den andern formen für lautgesetzliches $i$ ), schê opt. (doch auch skiêth, skiê durch anlehnung an skiâ(n), s. Gramm. § $272 \beta)$. Hingegen wurde im wfr. das $i$ bald ausnahmslos zur norm erhoben:

sg. ind. schŷt, schŷd S 384, 18. H 32. J 12, 21. 33. 19, 7. 28, 21. $60,1.68,1.75,8.76,1.7 .84,18$ (durch contraction aus

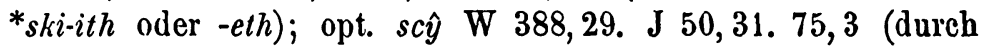
contr. aus *ski-e) und mit neuer endung skîe Sch 394;

wie auch im p. p. schŷn W, H 144. J 32, 4. 23. 62, 12. 64, 3. 5. 19. 78,3 etc. Sch 659 (aus *ski-in oder -en), woraus mit neuer endung schîen $\mathrm{S}$.

Eine spur der alten $e$-form begegnet aber noch in den sg. ind. scheed $\mathrm{J} \mathrm{3,2.62,15.}$

Formen mit $i \hat{a}$ aus $i a$ aus $i-a$ erscheinen in unsern quellen nicht mehr; dieselben wurden verdrängt durch schaen inf. J 12 , 32. 13, 47. 60, 4. 62, 12. 80,6. Sch 671 (nwfr. schean mit ea aus $\hat{a} \mathrm{GJ})$, schaet(h), schaed pl. W 475, 28. H 152. J 22, 11. 62, 12, neubildungen, die neben dem formell mit geet(h), steet(h) (s. oben s. 392) zusammengefallenen scheed nach dem muster von gaen, staen und gaet $(h)$, staet $(h)$ entstanden (s. a. a. 0.).

Eine alte starke praeteritalform blieb erhalten in scheen 'geschahen' H 167 (= aofr. aus dem sg. skê zu folgerndem *skên). Die regel bilden jedoch schîde, -en W 429, $27.436,2$. H 24. J 40, 4. Sch 601. 723 (nwfr. schŷdde GJ), schŷd W 436, 28. Rechtsqu. 486, 14. J 64, 7. 74, 2 (wegen der apokope vgl. oben zu quaen), schŷn $\mathrm{H} 24$ aus (schŷden, wie sioen aus sioeden, s. oben s. 393), entstanden aus dem nach skŷt gebildeten, aus nwfi. skŷde 'gescheben' GJ (mit epenthetischem $d$ ) ${ }^{1}$ ) zu folgernden skîa durch anschluss dieser formen an die schwachen verba $(b i)(h) l \hat{\imath} a$ 'bekennen' W, S, H und J passim, wîa 'weihen' $\mathrm{W}, \mathrm{J}$ 76, 1. 77, 9.2)

1) Der folge von falscher fassung des praet. schîde als schîdde, wie das $d$ in $\mathrm{nl}$. belijden, wijden, geschieden etc. aus belîen, wîen, geschien etc. (s. Franck, 'Taalkundige bijdr. 2, 165).

2) Auch in anderen dialekten finden sich von dem verb. schwache, auf ähnlichem wege entwickelte praeteritalbildungen: md. (vorzüglich ripuar.) geschiede, geschiet (wie gegiet, begiet p. p.; das ie aus $\hat{e}$ ), mnd. 
*Sl(i) t g 'träge'. S. oben s. 379.

Sneind(e). Im Wb. s. 1038 wird sniônd (sneuvend etc., s. oben 8. 355.361) mit sneind (snaind) identificiert und die ersterem zukommende bedeutung auch für letzteres angenommen. Doch ist sneyn im nwfr. der name für den sonntag und unterliegt die nämliche bedeutung auch fur die ältere sprache keinem zweifel, u. a. nach des wernsdeys ney Hwŷla Sneynd Sch 743 ( $\nabla$ gl. mhd. der wîze sun(nen)tac, mnd. witte sondach 'der sonntag Invocavit oder Judica').

Beztiglich der etymologie des nomens sei zunächst daran erinnert, dass das sn- von sniônd etc. auf einen alten, abnormalerweise nicht betonten compositionsteil *sunnan- oder *sonnen- etc. zurtickgeht ${ }^{1}$ ) und $e i$ (mitunter durch ai, ay dargestellt) vor nd (vgl. zu biriind) auf $e$, umlaut von $a$, beruht. Sodann aber bemerke ich, dass gegenuber -dag etc. in as. sunnandag, ahd. sunnantac, ags. sunnandaz, aofr. sunnandi, -dei, sonnendei und awfr. sonnendei $\mathrm{W} 438,16$, sônendei $\mathrm{W} 65,17$ ( $\mathrm{v} . \mathrm{R}$. falsch sonnen-). 403,14. H 92. 100.1142) -eind(e) ein jüngeres durch anspielung an ein charakteristicum des sonntages für -dei eingetretenes compositionselement sein muss; dass der ruhetag nach biblischer luberlieferung als das ende der arbeitswoche gelten konnte; dass der acc. snaynde Sch 534 auf einen ja-stamm hinweist; und dass das genus masculinum des wortes (op den snaynde, sneynd Sch 534. 727) mit dem genus von dij eynd finis $\mathrm{J} 18,10$, een eynten, eyndlycken, dyn eynd acc. sg. J 18, 2. 16. 19, 5. 24, 12 stimmt.

Stedde in J 7,1 so aegh dij sitter (angeklagte) ferst $X X$ daghen langh, dat hij hem byrêde, hôr hij in da rîchta [riacht]

schêde, geschêt (mit ê aus ie), mnl. geschiede, geschiet (wie (be)giede, begiet), deren ubertritt veranlasst wurde durch den umstand, dass die praesensformen in folge der contraction md. $\hat{e}$ aus $e(h) e$, as. anfrk. $i a$, ie aus $i-a$ für $e(h) a$ bez. $i(h) e$ gänzlich aus der norm der starken conjugation heraustraten.

Nwfr. hat auch im p. p. schŷd.

1) Die abweichende betonung der composita erklärt sich aus der neigung, den für die unterscheidung der beiden tagesnamen wichtigen zweiten compositionsteil hervortreten za lassen.

2) Umgebildet nach mônendey $W$ 390, 28. 31. 591,1 , wie umgekehrt monnen-, monnadeis W 389,6.10.15 (v. R. 6 und 10 falsch mondscipes, mondes). 390, 25 nach sonnendei. 
stedde, soe hij dan da oensprekera bycanne, als hyo [hy $\vec{a}]$ omcommen sint. Stedde steht als gegensatz zu bycanne $=$ 'die forderung anerkenne', muss also in der bedeutung 'die forderung bestreiten, es (mit dem kläger) aufnehmen' verwant sein. Das verbum begreift sich in solcher bedeutung ganz leicht als denominativ zu stede 'stätte, stelle'; vgl. mhd. staten c. dat. 'es mit einem aufnehmen, sich einem stellen', das sich zu dem auch als trans. verwanten staten, ahd. statôn locare verhält, wie fries. stedda $\mathrm{zu}$ an. steðja locare.

*Stiûg 'firmus', *stiûgia 'firmare'. S. oben s. 359.

Stoe und stoed 'stelle'. S. zu stwê.

Str.ŷdferde. S. oben zu freed fussn.

Stwê. Im 24. landrecht, wo tuber die strafe des brandstifters gebandelt wird, findet sich nach der awfr. sowie nach der aofr. Emsigoer ( $\left.E^{\prime}\right)$ version folgender zusatz: in W 79,14 ff. deer wr (ausserdem hat der uberfuhrte brandstifter) toe ieldane tri $\vec{a}$ merck dae liôdem om dine lioedferd [liuedferd], tri $\vec{a}$ merck da hellighem [hellighen] op dine frana alter om dine bannena ferd, tri $\vec{a}$ merck da schelta ende da aesgha om hiâra ban, ende hyâra st wê unslît ande sê; in $\mathrm{H} 96$ aldeer $n t$ [wr] ti ieldane tri $\vec{a}$ merck dae hollighum op dyn frâna altaer omme dyn bannena frede, tri $\vec{a}$ merc dae lioedem omme dyn lioedfrede, tria merc dae scelta ende dae aesga omme hiâre ban, ende hiare [hiàra] sibbe undslîtande sê; in $\mathrm{E}^{1} \mathbf{7 8}$,

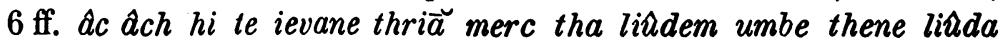
frethe, and tri $\bar{a}$ merc tha helghem te ievane oppa theth frâna altare te lidsane umbe thene bonnena fretha, and thri $\vec{a}$ merc tha asega anta schella têr of te ievane, thet hira bon unsclîtande (s. Aofr. gr. § 126) sê.

v. R.'s ubersetzung dieses unshitande durch 'ungestört' kann unbesprochen bleiben, denn aus $H$ ergibt sich, dass der erste teil des compositums und-, nicht die negation un- ist, mithin das $u n$ - in $\mathrm{W}$ und $\mathrm{E}^{1}$ als die durch assimilierung entstandene form zu gelten hat (vgl. die im Wb. citierten onberra, ondwaen, ongane, onnyela, onronnen, unswora, onswara, onfâ, onriachta etc. mit on-, un- fur ond-, und-, und s. fur das aofr. Gramm. § $122 \varepsilon$ ). Fiur die bedeutung des verbums ist mnl. (be)sliten = 'durch gerichtlichen spruch eine rechtsache zu ende fuhren' heranzuziehen. So begreift sich die betreffende stelle in $\mathrm{E}^{1}$ and thri $\vec{a}$ merc tha asega anta schelta têr of te ievane, thet hira bon un- 
sclîtande sê $=$ 'er hat drei $\mathrm{m}$. dem âsega und dem schelta deswegen zu zahlen, dass ihr urteilsspruch den process zu ende fuhre.'

In $\mathrm{W}$ wird dieselbe bestimmung etwas anders formuliert: 'er hat drei $m$. dem schelta und dem aesga zu zahlen von wegen des urteilsspruches und deren urteil (stwê) beende die rechtsache.' Stwê ist $=$ got. staua, ahd. stûa aus urgerm. *stôwô (Beitr. 17, 565): *stôrvô nom. sg., -ôz gen., - $\hat{o}^{n}$ acc. mussten nach bekannten lautgesetzen im vorfries. die formen $*$ stô nom., *stâa gen. acc. ergeben (mit $\hat{u}$ vor dunklem vocal aus analogisch entwickeltem $\hat{o}$ fur $\hat{o}-w$; vgl. awfr. dwaen inf., $\operatorname{dwaet}(h)$ pl. praes. ind. aus *dôan, *dôath und s. für dieselbe erscheinung im aofr. Gramm. $\S 20 \gamma$ ); aus dem acc. (und gen.) wurde dann durch secundäre schwächung des endungsvocals $-e^{a}$, woher *stûe $e^{a}$ das in folge der nämlichen accentverschiebung, welche bei $\hat{u} a>u \hat{a} \mathrm{zu}$ beobachten ist, zu siniê wurde (vgl. auch den opt. $d w \hat{e} \mathrm{~W}, \mathrm{~S}, \mathrm{H} 32$. J 15,16. 42. 25,39. 26,5 aus *dûe und die 1. sg. praes. ind. $d w \hat{e}$, dwee W 73, 35. H 94. Sch 517. 521. 537. 541. 543 etc. Ag 36. 37. 52. 64 etc. aus $* d \hat{u} e^{a}$ mit $-e^{a}$ aus der normalen flexion, welche diese endung fur $-u$ aus dem $-e^{a_{-}}$ (für $-i$-) der 2. 3. sg. entnommen hatte, und mit $\hat{u}$ nach analogie von *dûa(n), *duath). ${ }^{1}$ )

Nach der stelle in $\mathrm{W}$ ist das unsinnige sibbe in $\mathrm{H}$ als eine aus stwe verderbte lesart $\mathrm{zu}$ fassen, deren entstehung sich in graphischer hinsicht unschwer begreifen lässt.

$\mathrm{Sw}$ ommen, swommel in J 64, 8 Aan [Een] swommel dyeer jeffta $k w$ mey raeff bygaen ende neen raeff onder aldulke maneer: is dat seeck, dat een kw off een schet geet swommen in een oers länd ende ist hym leed, deer da kw off dyêr aegh ende drifft se wth, so ist schade [schada] ende neen raeff etc. Die beiden wörter sind als 'schwimmend (durch den grenzgraben)' zu

1) Auch die aus dem urspr. nominativ des subst. herrührende form (=ags. stón locus) war im awfr. (sowie im aofr., s. Gramm. §167) in schwang: stoe (stelle) W, H 64, op eenre stoe 'zugleich' J 84, 27, haudstoe 'eine kirche, die als der central- oder hauptplatz gilt für die daraus hervorgegangenen und dazu gehörigen kapellen', W, H 42,67. Neben stoe steht auch stoed locus W 426, 34 als compromissbildung aus stoe und sled; and ebenso haudstoed W, H 34, 104 neben haudstoe. 
deuten und beweisen als partt. praes. bez. verbaladjj. im verein mit der 3. sg. praes. ind. swomt $\mathrm{H} \mathrm{29}$. W 438,11 die existenz eines verbums swomma (nwfr. swomme GJ; vgl. auch wangerogisch swom, saterl. swumme, Ehrentrauts Archiv 1, 75. 2,181), das (mit $m m$ aus $*_{m n}$ ) zur kategorie awfi. ronna $\mathrm{H} 243$. W, S (nwfr. ronne im Hindeloper Zeemansalmanak, s. Halbertsma, Hulde aan G. Japiks 2, 202. 204), aofr. runnanda, burnt, burnath, spurna (Gramm. § 270 anm. 2), ags. spurnan, murnan, an. sporna, mnl. ronnen (Franck, Tijdschr. v. nederl. letterk. 2, 20) gehört und sich zur neubildung ahd. ags. as. swimman verhält, wie gedachtes ronna etc. zu got. ahd: as. rinnan, awfr. rynna $\mathrm{J} 60$, 17. W 75, 20 (nwfr. rinne GJ), ags. iernan, und aofr. burnt, -nath zu got. brinnan etc. Wegen awfr. $o$ für $u$ in hochtoniger silbe vor nasal vgl. hond, honger, iongh, pond, sonne, stomp, wonder, wonde etc. Die erbaltung des postconsonantischen $w$ vor altem $u$ ist naturlich der einwirkung der praeteritalform *swam zuzuschreiben.

In dem neben normalen winna, -en etc. W, S, H 164. 166. 170. J 13, 3. 15, 35. 72, 7 erscheinenden wonna, wont $\mathrm{H} 22.164$. 165 ist der wurzelvocal höchstwahrscheinlich nur die folge von analogiebildung nach ronna neben rynna und der neubildung bigonna, -gont $\left.\mathrm{H} 29.39^{1}\right)$ neben normalen biginna, -et, -e etc. $\mathrm{W}, \mathrm{S}, \mathrm{H}$ und $\mathrm{J}$ passim. Das nwfr. hat wonne Hindel. Zeemansalm. (Halbertsma, Hulde a. a. o.) neben winne GJ; das aofr. kennt nur winna (Gramm. $\$ 270 \alpha$ ), wangerogisch und saterl. winne (Ehrentrauts Archiv 1, 47. 2, 182).

1) Dass bigonna (sowie mnl. praes. begonnen) nicht als antiquität zu gelten bat, sondern nach analogie des praet. bigonde W, H 24, 168 (mnl. begonde, -ste) entstanden ist, geht hervor aus dem fehlen solcher bildungen in den agerm. dialekten (ahd. as. aofr.), denen ein praet. bigonda, -sta nicht fremd war. S. noch meine Mnl. gr. s. 222.

Die aofr. neben biginnath begegnenden biienne, begen, bigent (Gr. $\$ 270 \alpha$ ) sind keineswegs schwach flectierte formen (vgl. Beitr. 17, 306). Dies erweist schon das für $g$ eintretende $j$ angesichts des umstandes, dass altes $g$ vor secundärem palatalvocal erhalten bleibt (Gr. § $144 \alpha$ ). Das $e$ für $i$ kam hier auf durch anlehnung an das subst. biien (oder *bigen), dessen in schwach betonter silbe stehender wurzelvocal (man beachte die alte betonung von $b i$ - in nominaler composition) zu $e$ geworden war; vgl. aofr. bem sum, sent sunt, ende praep., Wideken F 58 (ahd. Witochind). 
Tauwe, tow 'geräte'. S. zu fliueswerp.

Thawa, tawa, -em, tâ, taewiane. Neben awane (um) kund zu tun (dass das uber den tuberfuhrten mörder verhängte urteil vollzogen werden wird)' W 413,17 1) (vgl. aofr. to auwande, $a(u)$ wed $\mathrm{zu}$ auwa $=$ ags. iewan und s. oben s. 376 anm. 1 und 377) begegnen das verb. thawa (d. b. tauwa) 'zeigen' (wegen th als schreibung furr $t$ s. oben s. 414 anm.) und das nomen tawa, -em (d. b. tauwa, -em) 'beweis, besitzdocumente': Hi (di dekken) scil êfler da kapittel sŷn breef thawa wr (vor) den hêla sindslael (sendgericht) Sch 375; Ende ief di ôra (dessen besitzrecht angefochten wird) haet dat awa [da tawa], so haet hi deer mey bihâlden sŷn bôdel ende sîn gued; ief him deert awa [deer tawa] brect, so aegh di oentingere een ferd (das recht der besitzergreifung) oen da gued W 398,14ff.; Aen aeft mey ma schêda mit tawen om quaedheed des wiues off dis mannis $\mathrm{J} 85,5 .{ }^{2}$ )

Mit riicksicht auf as. tôgian = got. ataugjan, as. getôkon aus *ataukôn und dgl. (s. Pauls Grundr. 1, 340, § 4) kann man schwerlich umhin, unser verbum auf *ataujjan zurtickzufuhren (wegen des nicht umgelauteten wurzellautes s. krawel, oben 8 . 378, und vgl. Aofr. gr. $\S 35 \beta)$ und das subst. tauwe als ein durch anlehnung an das verbum aus altem $\hat{\imath}-(j \hat{o}-) \operatorname{stamm} * a u w \hat{\imath},-w j \hat{o} z$ etc. entstandenes $\mathrm{zu}$ fassen; vgl. das offenbar identische -zowa in ahd. scrîbgezowa cautio (Graff 5, 713).

An der parallelstelle von $W 398,14$ ff., nåmlich $H$ 58, stehen für $d a$, deer tawa die doppelformen dae, dera $t \hat{a}$ : der alte auf *taunî zurltckgehende nom. sg. *lau ergab *tâ, wozu ein nom. gen. pl. $t \hat{a}$ aus $* l \hat{a} \cdot a(-0)$.

Als denominativ zum subst. findet sich ein schwaches verb. 2. klasse tauwia 'beweisen' in $\mathrm{H} 61$ Jef de man quêth, dat hi $z \hat{y} n$ eerva siald (verkauft) habbe..., so aegh mat him ti fulliane (zu ersetzen), ief hyt te taewiane habbe mey wirde ende mey buurscepe, deer hyt siuld habbe. Die parallelstelle W 400,8 hat hier ief hit mitter werde biwîsa mei.

1) N'u aegh aller scheltena lyc thi a wane binna sîne banne, dae brânden toe baernane ende dae kêdingha toe dwaen. An der parallelstelle $\mathrm{H} 138$ steht für thi awane verderbtes dis awendis.

2) Thavem in Nû wol ic den freede mey dughede sterkia ende fan treflika tha $w$ em nŷy riucht setta $\mathrm{H} 24$, ist ein ganz anderes wort, näml. dat. pl. zu thau 'gebrauch'; vgl. oben s. 377 und s. unten s. v. tref(te)lik. 
Têmende, têmed 'in ordnung'. S. zu hensich.

Tiaende 'in ordnung'. S. zu hensich.

Tioele 'tafel'. S. oben s. 358.

Tojênst-, to beckfŷnda 'durch gerichtliches erkenntnis zur beweisfubrung zulassen'. S. zu foerdwisa.

Tref(t)(e)lik, treftich, treft. Die wörter bedeuten nicht, wie das $\mathrm{Wb}$. angibt, 'zutreffend, trefflich' bez. 'bestimmung, festsetzung', sondern 'erforderlich, unentbehrlich' und 'arm' bez. 'behuf, notwendigkeit'; vgl. ahd. durft necessitas als fem. $i$-stamm aus *burfit, das nach fries. lautgesetzen treft ergeben musste (wegen der metathesis beachte frucht 'furcht', wrochte, wrocht, truch 'durch'). Die betreffenden stellen sind die folgenden: nu wil ick dyne ferd mit duegheden sterkia ende fan treflelycka tinghen (den erfordernissen gemäss) twâ nŷ̀ rî̀cht setta W 436, 15 (die parallelstelle $\mathrm{H} 24$ [s. oben anm. 2 auf s. 430] hat fan treflika thawen [unentbehrlichen gebräuchen gemäss] mit synkope des interconsonantischen $t$ ); Haet is der êwa riòcht? Eerlic thing rêda ende creftelic [treftelic] thyng biâdu (das erforderliche befehlen) H 21 (äbnlich J 1, 2; wegen der parallelstelle $\mathrm{W} 435,18$ s. oben s. 419 anm. 1 ); wirth hit (das vergehen des falschmunzers) folbrocht (erwiesen) 1) mit riûcht, so aeg di schelta dat gued to bisittane to heerna hând (in besitz zu nehmen, damit es dem magistrat zur verfugung stehe) ende dat treftiga (lies da treftiga nach der folgenden parallelstelle) weder to dêlane (es wider herausgebend unter die armen zu verteilen) W 422, $36 \mathrm{ff}$; (an der parallelstelle $\mathrm{H} 143$ steht needtreftich: ende mirt [wert] hi (der falschmunzer) mit tiôghe fulbrocht (uberfubrt)' '), soe aegh di scelta dat gued ti bisittane ti hêrana hând ende alle dâm, deer needtreftich sint, weder ti dêlane); deer sŷn tingh ief sŷn werf sêka schil om lainga (wegen vorladung) iefta om ielkirs treft des hêliga riachtes $\mathrm{W}$ 424, 7; Dat is riucht koninghes [keninghes] setma: dat is treft, dat met hallde da onriachte to pînen ende da riûchte liôdem to helpe W 424, 13; die parallelstellen $\mathrm{H} 147$ und 148 haben needtreft und neettreftich; in J 29,1 begegnet an einer mit W 424, 13 ubereinstimmenden stelle treftelick.

1) Vgl. mnd. vulbringen 'die wahrheit wovon erweisen'. In $\mathbf{H}$ wird das verbum metonymisch mit der person als subject verbunden. 
Tro(u)waden. Wie $\hat{a}$ vor $w \breve{o}$ zu $\hat{o}$ wurde (s. oben s. 378), entwickelte sich $a$ vor derselben lautverbindung $\mathrm{zu} o$, woraus in der folge ou: trouwaden 'trabten' zu *throw $\overrightarrow{0}$ - aus *thraw $\breve{o}-$ aus *thrav $\breve{o}-(\mathrm{vg}]$. mhd. draben aus *brabôn und s. wegen des $w$ aus $v$ [d. b. $\hbar$ ] oben s. 353) in dae hy (Octavianus) mey gôldene [gôldena] weynem toe Rome infoer ende by dae weynem trouwaden coninghen $\mathrm{H} 23$ (und W 436, 29, wo trowaden steht);

und sto(u)wia, -ath, -ade etc. 'staben', das sich ohne die Beitr. 17, 565 vorgeschlagene hypothese als rein lautliche entwickelung erklärt aus *stavő- (vgl. mhd. staben aus *stabôn); daneben stavia, -ie (d. h. stauvia) mit $a u-n$ aus $a-n$, dessen vocal in den flexionsformen mit -i- (d. h. $-i j$-) nach $n$ seine alte qualität erbalten hatte, oder = stavia, -ie (vgl. oben s. 363).

Die $n$ wfr. form des verbums lautet troaye ${ }^{1}$ ) (GJ und Epkemas einleitung zu G. Japicx s. LIX). Dieselbe steht auf einer linie mit scoya 'schauen', stoya 'staben' (nwfr. schoaye '), stoaye Beitr. 17, 566 sowie GJ und Epk. a. a. o.; oy, d. h. $\dot{o} i-j$, nach dem oben s. 346 über die einwirkung des nachrocalischen $j$ bemerkten aus $0-j$ bez. $\hat{o}-j$ für $0-n$ bez. $\hat{o}-n$ vor $-i-$ ), berechtigt mithin zur reconstruction eines awfr. *troya (aus *thro-wia $(n)$ mit analogischem $0-n$, wie das der bildung stoya zu grunde liegende *sto-wia(n)) als doppelform zu trouwia (aus *thro-wia(n) etc.; $o u-w$ oder $0-w$ aus den flexionsformen mit $-w \bar{o}-$-).

Zur kategorie scoya etc. gehört ferner noch nwfr. koaye 'kauen'1) (GJ und Epk.) = awfr. *koya als doppelform zu *kounia (beides aus *kôwia(n)). Fưr letztere formen ist mnl. kauwen heranzuziehen, das durch sein -auw- auf altes - $a w^{-2}$ hinweist (Beitr. 16, 306 ff.) ${ }^{2}$ ), also zur annahme eines vorfries. *kâwô- (und *kâwôja-) fuhrt, dessen wurzelvocal in der nach-

1) Daneben auch als doppelformen troagje, schoagje, koagje (d. b. nach jetziger aussprache tróaigje etc.) mit - gje neben -je nach analogie der mit beiden suffixen verwanten formen; vgl. die oben s. 399 verzeichneten awfr. verba mit -igia und -ia, sowie nwfr. achtgje, jachtgje, peynzgje, jeftgje 'beipflichten', kostgje etc. neben achtje, jachtje ètc. GJ.

2) Vor $w^{1}$ wäre nur ou möglich gewesen. Demnach muss auch das $w$ von ahd. chiuvvan (kou) und ags. céowan (céaw) als $w^{2}$ gelten und sind diese verbalformen als neubildungen zu fassen nach dem aus *kau (zu *kewan) hervorgegangenen praet. mit anorganischem, aus den andren flexionsformen entlehntem $w$. 
folgenden entwickelung wie das aus an hervorgegangene $a$ der vorstufen zu scouwia behandelt wurde.

Un(d)slítande. S. zu stwê.

Wrbecfŷnda, -wisa 'für ungulltig erklären, halten', 'durch gerichtliches erkenntnis zuletzt zur beweisfuhrung zulassen'. S. zu foerdwîsa.

Wrmynnia 'sich rerlieben in' in J 84, 3 Als een man een wŷff nael mey wrwynja [wrmynnia], eer hij se troune (lies trouwie) ende hij trouwie se dan mitta wirden (worten) ende mitter herta naet consenterie etc. Vgl. mnl. verminnen 'sich verlieben in' (s. Oudemans, Woordenb.).

Wrschelt, foerschôlt. W 425, 34 ff. (H 159): hueerso faeder ende een moeder lyaet (zeugen) tri $\breve{a}$ kŷnden, tween soenen ende een dochter, ende hâra ielderen dan stervet ende hĭ̌ (die tochter) dan een man nimpt bûta rêda bêdera brôren, soe quaet (sagen) da brôren, hiōhabbe her gued wrschelt, omdat hio et deen haet bûta hiâra reed; nw quêth her mônd, hiŏ nabbet naet wrschelt om dine frŷa wilker, deer her ende alla frowen di koningh Kaerl ghâf..., wantse agen wâld liues ende ledena and êne foermônd toe tziêsane. v. R. tubersetzt das p. p. ohne weitere erklärung mit 'verschleudert'. Es ist als 'eingebtisst' zu fassen zu * wrschelda $=$ ahd. fersculden 'einbüssen, verwirken'.

In $\mathrm{J} 87,1$ steht in der nämlichen bedeutung foerschôlt: (eine witwe, die innerhalb des trauerjahres wider heiratet, darf ibrem mann nicht mehr als ein drittel ihres vermögens vermachen und) da lyaefjefta (das witwengut), deer her byspritzen [byspritsen] (zugesichert) ende toeleyd sint fan her âra (ersten) man, ende da lawa (nachlass), deer fan sŷner wegen oenstoeren (als erbschaft zugefallen) sint, haet hio foerbeerd ende foerschôlt. Das part. gehộrt zu einem schwachen verb. 2. klasse * foerschôldia (vgl. ahd. gisculdôn, farsculdôn) und steht für * foerschôldet (aus *foerschôldat), wie die p. p. dwaeld J 12, 18 (2 mal), sigeld $\mathrm{J} 13,26.17,7$. 8, consenteert $\mathrm{J} 22,16$, oerloefft $\mathrm{J}$ 55,5 , bycommert $\mathrm{J} 20,17$, zu dwalia $\mathrm{J} 1,1$, syglia $\mathrm{J} 17,10.11$. 13. 26, 4, consenteria $\mathrm{J} 12,26$. 84, 3, *oerlovia (p. p. oerlovet $\mathrm{J}$ $55,5.6)$, bycomria $\mathrm{J} 43,11$.

Wrscet 'verbindert'. S. zu wrstôrde.

Wrstôrde. In W 43, 2 wird die zweite der im ersten landrecht erwähnten needschŷn (der gesetzlich anerkannten ver- 
hinderungen, den gerichtstermin einzubalten) folgenderweise formuliert: dat him sŷn fîande (lies fianden)dine vei wrstôrde. Die aofr. parallelstellen baben statt dessen thet him sin fiand thene wî ûr stôde (vertreten hätte) $\mathrm{R}^{1} 43,5$; thet him sin fînd thene wei ... arstôde Fiv. 22; theth him sin fiund thene wey ar stonde $\mathrm{E}^{2}$ (s. Rq. 40, no. 8). Mit rücksicht auf dieses $\hat{a r}$ stôde, -stonde läge es nahe, die lesart von $\mathrm{W}$ als eine verderbte gelten zu lassen und in wrstôde zu corrigieren. Doch wäre die berechtigung dazu sehr fraglich, denn die möglichkeit eines wrslôrde = 'gestōrt bätte' (mit vei = 'reise') lässt sich nicht in abrede stellen: zu ârstêra 'zerstören' J 64, 14, ursteert 'verhindert, macht aufbören' W 434,21. 435, 7, tystêrth $\mathrm{H} 22$ und foerstêrath (lies foerstêrth) H 177 mit gleicher bedeutung gehörte ein mittelvocalloses praeteritum stoerde, das sich u. a. auch findet in tostoerden 'zerstörten' W 437, 30, tostoerde 'hob (die gesetze) auf' W 429, 13 (vgl. auch nwfr. forstoard 'verstört, verstimmt' GJ), neben unurspritinglichen tostêrden 'zerstörten' $\mathrm{B}$ 27, toesteerde 'hob auf' H 166.

Statt wrstôrde hat die parallelstelle $\mathrm{H} 83$ wrschet: Dat him sŷn fianden den wey wrschet habbe. Fưr das hieraus zu folgernde wrschetta $=$ 'verhindern' vgl. ende hot soe ioe ti sanne falt (in welcher sache ibr, richter, zum streit, zum widerstand verpflichtet seid) iêns den biscop ief yêns den decken iefta toeyênst ênige hauden, dêr (die) ws ... ponten wol bihŷndrya iefta fornedrya ..., dat ghŷy dat scette mith rêde ende mit dête $\mathrm{H}$ 301 und datler schillet vessa alle wettergongen schet (abgedämmt) etta bannena dîke W 419,29 (H 128); und beachte aofr. sketta '(blut) zum stehen bringen' nebst skettene 'das einsperren von vieh' (Gramm. § 286 ß. 176. Beitr. 17, 336).

Wt(b)a. Nach der W $412 \mathrm{f}$. fur die mordklage vorgeschriebenen processordnung soll der grewa im verein mit seinen beisitzern dem angeklagten die anforderung stellen zu erklären (Nâ quêth him di grewa toe ende wîsa lioed) dat hi deer mede ne weer an ferthe ${ }^{1}$ ) nêr an fulliste (hilfsleistung) nêr

1) Aus *giferthi $=$ mnl. geverde ntr. 'geleite', nicht nach $\nabla$. R. zu ferth 'fahrt, reise' gehörend (th als rest alter orthographie für $d$ ). An der parallelst. $\mathrm{H} 136$ steht firde. Wegen $i$ und $e$ als wechselschreibungen zur darstellung eines ie aus $e$, umlant zu $a$ oder $=$ altem $e$, vgl. bihirda 'erbärten' W, H 114, hirda 'antreiben' J 60, 3 ( Da tyêtwen mey ma helpa 
fîchlende, da ma dine man sloegh binna da alra hâgista frede. ${ }^{1}$ ) Dann wird dem angeklagten vom aesgha die anforderung gestellt, dat hi da sikeringha habba scil (zum zeugnis fur seine schuldlosigkeit vorbringen soll) ${ }^{2}$ ) dine schelta, deer him dae ban wr lâth (der ihm die vorladung zugestellt hat), ende dera tôlva saun (und die grössere hälfte dessen 12 schöffien). Wenn ihm dieses mittel sich pon der anklage zu reinigen $\mathrm{zu}$ gebote steht, dann sollen die erwähnten beamten bezeugen, dass or nicht zugegen gewesen sei, als der mann erschlagen wurde. Wenn ihm aber dera wtha breckt, dat is biwyss, deer him di aesgha wŷsd [wiisd] haet, soe schil hi mitter haudlêsene bêta.

Was hier mit wtha (in $\mathrm{H} 173$ ata; wegen th als schreibung fur $t$ s. oben s. $414 \mathrm{anm}$.) gemeint ist, liegt auf der hand: 'der beweis des alibi'. Wir haben somit das nomen als ein aus $a t$ und dem oben s. v. thawa besprochenen als plurale tantum verwanten $t \hat{a}=$ 'beweis' gebildetes compositum zu fassen, dessen zweiter teil aber, wie aus der schreibung mit einfachem $t$ hervorgeht, in folge seiner schwachen betonung verktirt wurde und, indem sein vocal so mit der endung des nom. acc. gen. pl. nach der $\hat{o}$-flexion zusammenfiel, dem substantiv das gepräge eines derivatums zu $\hat{u} t$ verlieb.

- nyôgenderhânda nôys toe der-lyeffle; mit-onthaldene ende-myt deelnyminghe, mit to hirdane, mit reed ende mit tohinsinge etc.; vgl. mnd. herden 'antreiben') und herde 'arbeit' W 437, 13 (an der parallelst. H 25 steht arbeyd); $\hat{u}$ (firdich 'fortziehend' W, riùchtfirdich(eet) J 2, 11. 16. 14, 5. $24,7.36,15.46 .49 .55,4$, riùchtfirdigia $\mathrm{J} 21,8$ und $\hat{u}$ (ferdich $\mathrm{H}$ 141, riüchtferdich(eet) J 27, 4. 5, lioed-, liuedwirden 'busse wegen bruchs des volksfriedens' (volksverletzung) W (beachte as. Awerdian 'zersturen') und lioed-, liudwerden $(e) \mathrm{S} 446,1.24 .28 .455,8.31 .463,7.499,38 ;$ swird $\mathrm{W}, \mathrm{H}$ 31. 46. 113. 143. J 13, 24. 26, 10. 30, 5. 72, 4. 86, 5 und swerd $\mathrm{S}, \mathrm{H}$ 144. 179; hird 'herd' J 50, 38 und herdsteed, -staede W, H 96; stirt cauda W und stert $\mathrm{H} 112$; wird dignus $\mathrm{W} 392,27.387,36$. J 33,17, wird 'wert' (subst.) W 390, 1. 3. 33. 391, 25. 4i6, 7. S 489, 21. H 35. 36. 301 und werd adj. H 30. 95, werd, werth (subst.) S 498, 33. H 36. 95, werde H 152. 154; clirck J 58, 32. 65, 15. 80, 4. 81, 23 und clerck J 55, 5 etc.; vgl. nwfi. (GJ) hirde 'aushalten', riuechtfirdich, swird, hird 'herd', stirt, wird dignus, klirck etc.

1) An der parallelstelle $\mathrm{H} 136$ steht Nô sprect him di grieuwa toe ende wîse lioede, dat hy deer mey weer [wère] an firde ende fulliste ende fiochtende, da man dyn man sloegh binna dae allerhagista ferda, d. h. der grieuwa etc. behaupten, er sei zugegen gewesen etc.

2) Die parallelst. H 137 hat dat hi ti dae sikringem habba schel etc. 
Das nämliche wort erscheint noch an einer anderen stelle in unseren gesetzen, in W 394, $34 \mathrm{ff}$. (H 46), jedoch in der etwas abweichenden bedeutung 'das recht den beweis zu führen, dass man zu einem andern gerichtsbezirk gehört': als ma dyn frîa Fresa to strîde tinghia wil ende di ôra (der geforderte) da wta habba wil, dat hi binna trim degem comma schil, ief hi da wta habbe wil; ief ma him mitta strŷd [striid] bigre $\breve{e} t$, so schil hi sprecka ende sidsa, hi ne thoer bi. dis schelta ban hôr iechtwird nêr seckwird dwaen nêr neen tinger ontfaen, omdat hy deer huis nêr hof haet.

Wâya. S. zu grôya.

We(i)nden. In den aofi. busstaxen begegnet widerholt eine alliterierende aufzählung der verschiedenen verrichtungen, woran einer in folge von verletzung verhindert werden könnte. So in $\mathrm{R}^{2} 536,27 \mathrm{ff}$. Thet hi sa wel wesa ne mî (kann) an bethe (bade) an bedde, ne an wigghe ne an wayne, inna wîe (wege) an wetire, ne an huse ne an godishuse, ne mith sînre wîve, sa hi êr machle, ne bi sîne fiôre ni an nênre ferde him sa wel bithancka mî, sa hi êr machte; s. ferner $\mathrm{R}^{1} 85,23 \mathrm{ff}$. H 84, $16 \mathrm{ff}$. 332,9 ff. E' 85, 23 ff. 226, 2 ff. E' 226, 5 ff. F 62 mit mehr oder weniger ähnlicher formulierung. An den awfi. stellen, wo sich diese aufzählung findet, steht anstatt an wigghe (widze) ne an wayne die formel an wîghen nêr an wenden $\mathrm{S} 450,16$, an wîge nêr an weinden $\mathrm{W} 465,6$, an wîghe nêr an wendum $\mathrm{H}$ 229, und zwar offenbar als neuerung in folge des umstandes, dass das alte ausser brauch gekommene wigghe 'pferde' nicht mehr verstanden wurde: indem wîghen (pugnis) oder wîge (pugna) an die stelle von wigghe trat, konnte weyne (vehiculo) als nicht in der verbindung passend empfunden und durch ein $\mathrm{zu}$ nenda 'sich wenden, bewegen' gebörendes verbalsubstantiv auf -en (= got. -eins) ersetzt werden. Wegen des wurzellautes von we(i)nden s. oben zu biriind.

Wennia, wan(n)ia. Das zeitwort begegnet u. a. als inf. und ger. wennia, -ie, -yan J 37,11. 72,4. Sch 671, als p. praes. wennien J 42,3. 4, als 3. sg. praes. ind. wenneth, -at J 15, 23. Sch 629 ( . R. falsch wenat) (nwfr. wenje). Der Beitr. 17, 346 anm. vorgescblagenen fassung desselben als eines ursprtinglich zur ai-klasse gehörenden und nach art von as. hebbian, habbian, habas, -ad, seggian, sagis, -ad, ags. libban, liofast, -að etc. flec- 
tierten verbums wage ich nicht beizupflichten wegen der durchgehenden conjugation nach 2. klasse; vgl. gegenuber wennia etc. mit wenneth, -at, libba, -en, -at, -et $(h),-e \mathrm{~W}, \mathrm{~S}, \mathrm{H}$ 28. 48. 54. 87. J 36, 14. 44, 2. 46, 25. 50, 37. 56, 2. 83, 1. 87, 6 etc. mit der 3. sg. praes. ind. liwet( $h) \mathrm{W}, \mathrm{J} 44,2.50,40.44$. 73, 1, liuwat(h), -eth $\mathrm{H}$ 32. 55. 60. 94. J 87, 5 (vgl. oben s. 359 und 363), habba, -en, -e, -et etc. W, $\mathrm{S}, \mathrm{H}$ und $\mathrm{J}$ passim, hebba, -ane, -e $\mathrm{H} 81$. 158 mit haest(e) W, S, J 6, 7. 15, 54, haet(h) uberall passim. Wir haben m. e. an ein von haus aus zur 2. klasse gehörendes denominativ zu denken, dem ein subst. $*_{n e n}(n)$ (ntr. $j a$-stamm) oder *wenne (jô- oder jôn-stamm), aus * wunj- zu grunde liegt, dessen frühere existenz erwiesen wird durch ein nach art von lyffheftich Sch 724, strîdheftich W, wîedheftich $\mathrm{H}$ 105, eerhaftich, -heftich J 15, 77. 19,4. 56, 5, leynafftig J 56,6, stanaftich (lies stândaftich) J 44,18, schaedaftich J 82, 13, -achtich J 64, 4, ontyenstaftich $\mathrm{J} 44,16$, (on)deelachtich $\mathrm{J}$ 25, 14. 56, 1, boefachtich J 37, 7 mit einem subst. componiertes wenheftich, -haflich Sch 354. 629. Ag 18. 62. 136, -aftich, -achtich J 20,1.6. Ag 110.

Neben wennia stehen auch wannith, -ath, -ede Ag 102. Sch 535. S 511, 19 (nwfi. wannyen Ag 257) und waenyen Ag 126 als analogiebildungen nach hallia, bitallia, (bi)warria, mackia und halia, bitalia, (bi)waria, makia neben hellia, bitellia, (bi)werria, - meçkia .(s, ọbẹn. s. 34̣8f. f.):

Wilinga. v. R. las W 401, 21 unrichtig wiliga. Die an sich schon verdächtige deutung des wortes als umstellung von wigila (Wb. 1149) wird also hinfällig. Das wort = 'hexerei' entspricht ags. nizelunz incantatio, geht also mit ubergang des postrocalischen $g$ vor $l$ (vgl. neyl 'nagel', wio brein 'hirn', wein 'wagen', reyn 'regen' etc.) und absorption des cons. auf * wr̂gling'zurïck'.

Wrichte 'arbeiter' in $\mathrm{J} 37,8$ Als een menscha wonnen haet een wrichte ende dat waer (wetter) foerkeert off reynth, alzo dat hij sŷn wirck naet dwaen moge ..., so aegh hij sŷn laen allŷck fol ney der $t \hat{y} d$, hitten sê dat hij wonnen sê to onbescâtte $\left.{ }^{1}\right)$ wirck. Das nomen ist identisch mit as. wurhteo, ags. wyrhta. Bezüglich seines $i$ ist folgendes zu beachten.

1) Wegen onbescât 'unbestimmt' vgl. bischat 'bestimmt' in Hwaso orkenschip fêra schel, dij schel frij wessa ende to byscatta jêrem 
Während der aus $\ddot{u}$ hervorgegangene laut normal durch $e$ dargestellt wird, erscheinen vor tautosyllabischem $r, l$, vor $n n$ und $n+$ guttural oder hieraus entwickeltem assibiliertem laut meist abwechselnd $i$ und $e$ ( $i$ im grossen ganzen häufiger als $e$ ), ein umstand, der auf eine zwischen $i$ und $e$ liegende qualität des vocals vor besagten consonanten, also auf $i^{e}$ schliessen lässt 1):

wirtsa, -en, -e W 2), H 31. 125. J 37, 8, wirka H 125 (ags. wyrcan) und werkande $\mathrm{H} 118$; kirta 'kürzen' $\mathrm{W}, \mathrm{H} 25 . \mathrm{J} 8,2$ und kerta H 26. 162. 167. 169; wird(e) fem. 'zeugnis, wahrheit' W.2) H 2. 22. 30. 125. 128. 133. 175. 176. 302. J passim (got. -waurdei) und werde $\mathrm{H}$ 93. 1073); forwirde 'contrakt, bedingung' $\mathrm{J} 15,1$. 2. 82, 13. 84, 20, forwirte mit gleicher bedeutung $\mathrm{J} 37$, 1. 4. 41, 34) und forwerda $\mathrm{W} 395,22$; andwirde und andwert, andwerdia (s. oben s. $407 \mathrm{anm}$.); wird 'wort' (der umgelautete vocal durch anlehnung an altes *werde 'bewortung' $=$ aofr. werde Beitr. 14, 276; wôrd begegnet u. a. H 135. 175), iecht-, seck-, grêt(wird $\left.\mathrm{W}^{2}\right), \mathrm{H}$ 22. 53. 107. 109. 120. J passim und werd, weder-, iecht-, seck-, grêtwerd H 20. 28. 29. 39. 40. 44.

$\mathrm{J} 15,29$; Hweerso mônd schil wesse joff een foerwarer der ongêrighe $k \hat{y} n d e n, d \hat{y}$ schil wesse to byschatte jeren, als fîf ende tweyntich jeer ald J 26, 9; Hwaso ôrem schylda qwŷt leet .... dat mey een man weerrôpa ... ende dat om be schatle secken $\mathrm{J} 44,7$; di dekken dae dekkenŷe toe ontfaen fan thae biscope... sonder kape en sondir by schatte pachte Sch 375. Das p. gehört zu biskêda (s. oben zu kĕttich).

1) Vor $n+z(\mathrm{~d}$. h. $t s$ ) oder $s$ steht $e$ oder $e i$ (ai) beides $=e i$ (vgl. oben zu biriind): enze, -se $\mathrm{H}$ passim $\mathrm{S}$, einze, -se $\mathrm{W}, \mathrm{H} 48, \mathrm{~J} 59, \mathrm{i} .16$, ainse $\mathrm{Ag} \mathrm{87,} \mathrm{S} \mathrm{(ags.} \mathrm{yndse).} \mathrm{Man} \mathrm{beachte} \mathrm{hierneben} \mathrm{nwfr.} \mathrm{(GJ)} \mathrm{peynzje,}$ feynzje $=$ mnl. peinsen, pensen 'bedenken' (ags. pinsian pensare), veinsen, vensen.

2) Einzelne im Wörterbuch stehende citate sind nicht beweisend, weil die incunabel daselbst den vocal $+r$ mit der abbreviatur' (dio auch für er verwant wird) bezeichnet. Wo die betreffenden formen in $H$ und $\mathrm{J}$ mit' begegnen, citiere ich dieselben nicht.

8) Fir v. R.'s werde $\mathrm{W} 400,8$ steht in der incunabel $w^{\prime}$ de. Die Beitr. 14, 277 vorgeschlagene annahme einer beeinflussung des nomens durch wêr verus ist nicht stichhaltig, weil die bedeutung 'wahrheit' sich auch ohne solche einwirknng aus 'zeugnis' entwickeln konnte.

5) Die form mit $t$ ist zurïckzuführen auf *forwürdithu, deverbativ zu *forwürdjan (vgl. mnd. vorworden 'pactieren'). 
45. 46 (Hett. falsch seckivird). 53. 107. 109. 110. 120. 122.134. 135. $\left.136^{1}\right)$;

gilden 'gtilden' H 65 und gelden $\mathrm{H}$ 23. 98. 106; hilde 'buld' H 127 und helde H 127; schild 'schuld' H 67. 145. 173. 302. 303. J passim. Sch 511. 535. 537, schildich H 100. 303. J passim. Sch 522. 533. 543. 645 und scheld H 23. 59. 63. 73. 84. 101. 122. 123. 180, scheldich $\mathrm{H}$ 120. 127, twiscelde $\mathrm{H} \mathrm{74,}$ wrscelt (s. oben s. v.); schil, schillat $(h)$, -et(h) W, H 2. 34. 35. 37. 119. J passim und schel $W 387,24$. 389, 4. 394, 23. 405, 15. 406, 25. 407, 26. 409, 21. 412, 18. 24. 26. 415, 37. 434, 28. 435, 21. 29. 34. 438,7 . 14 . 16 etc. $\mathrm{H}$ und $\mathrm{J}$ passim, sel $\mathrm{W}$, schellat $(h),-e t(h)$ W 19, 18. 389, 11. 390, 24. 391, 5. 393, 8 etc. $\mathrm{H}$ und $\mathrm{J}$ passim;

kinne genus $\mathrm{W}$ und kenne $\mathrm{H} 49.159$; in-, wlryn 'das hinein-, hinauslaufen' $W$ und inrenne $S$; synna 'sonne' $\mathrm{H} 3$ und senne H 118. 162. 164. 180;

tynse 'dünke' W, t(h)inckt, thincketh 'däucht' J 81, 4. Sch 662. H 152 und tenket Sch 528.

Neben blôd-, wtstirtinghe W, J 76, 1.6. 7, (nt)stirt(h) p. p. $\mathrm{W}, \mathrm{H}$ 108. J 76, 2, wŷrm 'wurm' J 81, 23 (wegen des gedehnten vocals vgl. nwfr. wîrm), willen laneus $\mathrm{W}$, H 179, clinna, -e, -en 'klingen' W, H 74. 148 (ags. clynnan), klin 'klang' J 59, 18, berschinse W und bereschinke H 96 (wegen des wortes s. Aofr. gr. $\$ 308$ ) begegnet kein beleg mit $e$, offenbar weil in diesen wörtern der vocal auf seinem entwickelungsweg von $e \mathrm{zu} i$ das $i$-stadium schon erreicht hatte; im $n w f r$. haben ja alle die oben verzeichneten formen, insofern dieselben noch in schwang sind, $i$ : so nach GJ nicht nur stirte, sondern auch wirtsedey 'werkeltag', kirt adj. (durch anlehnung an das verb.), wird, hilde, schild, sil, wille, -en, sinne, tinsje, tinkt und ausserdem in der jetzigen sprache (wo tibrigens das ir vor dental mit synkope des $r$ als das $u$ im engl. put, butcher, pull, run etc. lautet) wîerm, tin (awfr. *tinne, *terne 'dunn').

Constantes $e$ weisen hingegen auf: berthe, -te, -de 'geburt', berthe, bernt(h)e, berdene 'last' (s. fur die belege oben s. 414 anm.), $\operatorname{verp~} \mathrm{W}, \mathrm{S}, \mathrm{H} 20.42 .122 .156$ (ags. wyrp), deren vocal sich begreift als die folge von anlehnung an das p. p. bern

1) Für v. R.'s werd $\mathrm{W} 63,14.403,9$ steht in der incunabel $w^{\prime} d$. W 411, 29 hat wird (nicht werd). 
'geboren' W, S, H und J passim (mit junger synkope aus *beren), (op)berra 'heben' J 33, 13. 37, 14. 47, 3. 12. 50,31. 52, 1. 68, 3. 77, 4 (mit junger gemination, ahd. burien), werpa;

und herne 'winkel' W, H 96 (nwfr. herne GJ), schelta 'schulz' uberall passim, wennia 'wohnen' (s. oben s. v.), wo das $-a(-)$ der folgesilbe die entwickelung des wurzelvocals nach der $i$-richtung verhinderte (nämlich in der flectierten form herna gen. dat. acc. sg. nom. acc. pl., in schelta nom. gen. dat. acc. sg. nom. acc. pl., und wennast, -ath, -ade, -ad), sowie o(e)ngert 'ungegurtet' W, H 56 mit $e$ in folge von generalisierung des ursprünglich nur dem inf. und plur. praes. ind. auf - $a$, -ath zukommenden vocals (im gegensatz zum $i^{e}$ bez. $i$ in wirtsa, kirta, *stirta, wo der in den andern flexionsformen entwickelte laut verallgemeinert wurde) und das an *gerda angelehnte gerdel 'gürtel' W, J 58, 30. H 163; vgl. noch nwfr. (GJ) scherte 'schlirze, schooss' mit $e$ aus den casus auf $-a$ (an. skyrta, $-u$ 'hemd') und schirte mit $i$ aus dem nom. sg. auf $-e$. (Der vocal des einmal belegten ferdria 'fördern' H 303 entzieht sich der beurteilung; nwfr. gilt beforderje GJ).

Vor cht blieb $e$ erhalten: drecht 'schaar' W, H 87. J 50, 41, flechtich 'flüchtig' W, H 162. J 12, 23. Demnach ist der' wurzelvocal von wrichle nicht vor cht, sondern in der vorstufe der uberlieferten form *wierchta entstanden (wegen der metathesis vgl. oben zu treftelik). Zwar hätte hier nach herne etc. lautgesetzlich das $e$ haften bleiben mussen, doch konnte dasselbe durch anlehnung an wirtsa, wirka etc. gar leicht durch $i^{e}$ verdrängt werden.

Wrichten, -a in W 393,6 (H 43) ief di frîa Fresa toe strîde bitinget wirth ende hi quêth, dat hi aynlikes guedes soe fula oen sŷnre wer (besitz) naet habbe, dat hi wêpen ende wrichten (in $\mathrm{H}$ wrichta) winna moge. $\mathrm{v}$. R. ubersetzt das wort durch 'kampfkleid' (wol besser 'kampfkleidung' wegen der pluralendung -en bez. -a) und zieht ein (mir unbekanntes) ags. wrigan vestire heran. Es ist vielmehr ags. wréon aus *wrîhon ins auge zu fassen, wozu das nomen als deverbatives mit - $t \hat{o}$ gebildetes derivatum ( gl. Kluge, Nom. stammbild. § 120) gehört; also eig. = 'schutzkleidung'. 